\title{
Detection of the Presence of \\ Long Range Dependence in Time Series
}

by

\section{Gennadi Gromykov}

\begin{abstract}
A thesis submitted to the Faculty of Graduate and Postdoctoral Affairs in partial fulfillment of the requirements for the degree of
\end{abstract}

Master of Science

in

Probability and Statistics

Carleton University

Ottawa, Ontario

(C) 2011

Gennadi Gromykov 


$\begin{array}{ll}\begin{array}{l}\text { Library and Archives } \\ \text { Canada }\end{array} & \begin{array}{l}\text { Bibliothèque et } \\ \text { Archives Canada }\end{array} \\ \begin{array}{l}\text { Published Heritage } \\ \text { Branch }\end{array} & \begin{array}{l}\text { Direction du } \\ \text { Patrimoine de l'édition }\end{array} \\ \begin{array}{l}\text { 395 Wellington Street } \\ \text { Ottawa ON K1A ON4 } \\ \text { Canada }\end{array} & \begin{array}{l}\text { 395, rue Wellington } \\ \text { Ottawa ON K1A ON4 } \\ \text { Canada }\end{array}\end{array}$

Your file Votre référence

ISBN: 978-0-494-81697-4

Our file Notre référence

ISBN: $978-0-494-81697-4$

NOTICE:

The author has granted a nonexclusive license allowing Library and Archives Canada to reproduce, publish, archive, preserve, conserve, communicate to the public by telecommunication or on the Internet, loan, distribute and sell theses worldwide, for commercial or noncommercial purposes, in microform, paper, electronic and/or any other formats.

The author retains copyright ownership and moral rights in this thesis. Neither the thesis nor substantial extracts from it may be printed or otherwise reproduced without the author's permission.
AVIS:

L'auteur a accordé une licence non exclusive permettant à la Bibliothèque et Archives Canada de reproduire, publier, archiver, sauvegarder, conserver, transmettre au public par télécommunication ou par l'Internet, prêter, distribuer et vendre des thèses partout dans le monde, à des fins commerciales ou autres, sur support microforme, papier, électronique et/ou autres formats.

L'auteur conserve la propriété du droit d'auteur et des droits moraux qui protège cette thèse. $\mathrm{Ni}$ la thèse ni des extraits substantiels de celle-ci ne doivent être imprimés ou autrement reproduits sans son autorisation.
In compliance with the Canadian Privacy Act some supporting forms may have been removed from this thesis.

While these forms may be included in the document page count, their removal does not represent any loss of content from the thesis.
Conformément à la loi canadienne sur la protection de la vie privée, quelques formulaires secondaires ont été enlevés de cette thèse.

Bien que ces formulaires aient inclus dans la pagination, il n'y aura aucun contenu manquant. 


\begin{abstract}
The aim of the thesis is, first, to penetrate into the relatively new advanced theory of long memory time series and, second, to attempt improving some existing methods. We discuss selected techniques for detecting the presence of long range dependence in time series, and introduce the preliminary test estimator with adaptation to the detection problem of interest. We also apply the maximal total error probability criterion to compare the performance of various statistical tests. In addition, we closely consider proofs of several recent results in this area and fill in some omitted details.

The estimation and testing techniques considered in the thesis are supplemented with simulation results. The $\mathrm{R}$ codes used to run simulations are given in the Appendix.
\end{abstract}




\section{Acknowledgements}

I would like to thank my supervisor Dr. Mohamedou Ould Haye for his support, guidance, and encouragement during the preparation of this thesis. I express special thanks to Drs. Rafal Kulik, Majid Mojirsheibani, and Chi Wan for serving on my graduate committee and for making comments and suggestions that helped to improve the final version of the thesis.

I am especially grateful to my wife for her patience, love, and encouragement. 


\section{Contents}

1 Introduction 1

1.1 Notion of Time Series . . . . . . . . . . . . . . . . . . 1

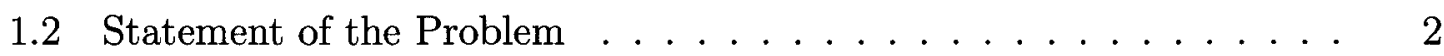

2 Preliminary Information $\quad 5$

2.1 Short Memory Models and Their Properties . . . . . . . . . . . . 5

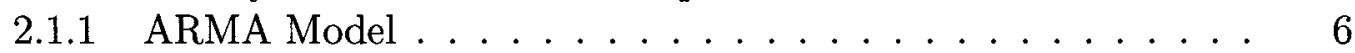

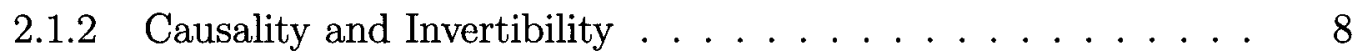

2.1.3 ARIMA Model . . . . . . . . . . . . . . . . 10

2.2 Motivation of Long Memory and Models . . . . . . . . . . . . 12

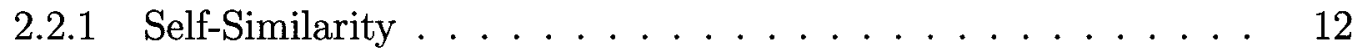

2.2.2 Fractional Brownian Motion . . . . . . . . . . . 15

2.2.3 Definition of Long Memory . . . . . . . . . . . . . . . . 17

2.2.4 Fractional Gaussian Noise .............. . . . . . 18

2.2 .5 FARIMA Model . . . . . . . . . . . . . . 19

2.2.6 Spectral Density of FARIMA ............. . . . . . 21

2.3 Some Important Concepts in Convergence . . . . . . . . . . . 25

2.3.1 Convergence in $L_{2}[0,1] \ldots \ldots \ldots . \ldots . \ldots 25$

2.3.2 Donsker Type Theorems ............... . . . . 26

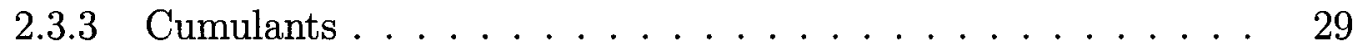

2.3.4 $O_{P}(\cdot)$ and $o_{P}(\cdot)$ symbols $\ldots \ldots \ldots 31$

3 Detecting Long Memory through Estimation 33

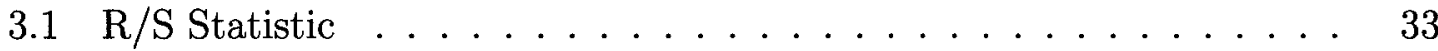

3.2 Variance Plots . . . . . . . . . . . . . . . 36

3.3 Geweke and Porter-Hudak Estimator . . . . . . . . . . . . . 39

3.4 Whittle Estimator . . . . . . . . . . . . . . . . . 41

4 Detecting Long Memory through Testing $\quad 47$

4.1 Modified R/S Statistic . . . . . . . . . . . . . . . . . . 47

4.1.1 Definition of Modified R/S Statistic . . . . . . . . . . . . . . 47

4.1.2 Testing Short Memory versus Long Memory Using Modified

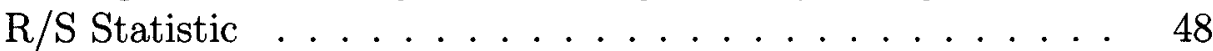

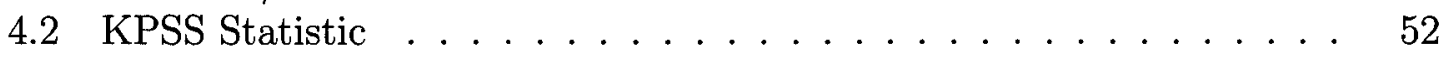

$4.3 \mathrm{~V} / \mathrm{S}$ Statistic . . . . . . . . . . . . . . . . 55

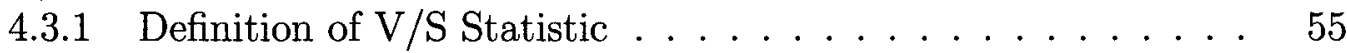

4.3.2 Asymptotic Behavior of V/S Statistic . . . . . . . . . . . 55

4.4 Increment Ratio Statistic . . . . . . . . . . . . . . . . . . 70

4.5 Preliminary Test Estimator . . . . . . . . . . . . . 71 
5 Comparison of Tests and Empirical Studies $\quad 75$

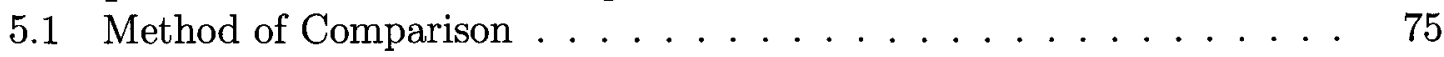

5.2 Short Memory versus Long Memory . . . . . . . . . . . . . 75

6 Conclusion $\quad 81$

$\begin{array}{ll}\text { Appendix } & 82\end{array}$ 


\section{List of Tables}

1 Conditions for stationarity, invertibility, and causality in ARMA models. . . . . 9

2 Quantile values of the limiting c.d.f. of the modified $R / S$ statistic. . . . . . . . 49

3 Quantile values of the limiting c.d.f. of the KPSS statistic. . . . . . . . . . . 53

$4 \quad$ Quantile values of the limiting c.d.f. of the V/S statistic. . . . . . . . . . . 58

$5 \quad$ Updated quantile values of the limiting c.d.f. of the $V / S$ statistic for $d=0 . \ldots 60$

6 Total error probabilities of the modified R/S, KPSS, and V/S tests, in percentage. 77

$7 \quad$ Empirical size of the modified $R / S$ test, in percentage. . . . . . . . . . . . 78

8 Empirical size of the KPSS test, in percentage. . . . . . . . . . . . . 78

9 Empirical size of the $\mathrm{V} / \mathrm{S}$ test, in percentage. . . . . . . . . . . . . . . . . 78

10 Empirical power of the modified $R / S$ test, in percentage. . . . . . . . . . . 79

11 Empirical power of the KPSS test, in percentage. . . . . . . . . . . . . 79

12 Empirical power of the V/S test, in percentage. . . . . . . . . . . . . . . 79

13 Empirical size of the 5\% test based on the $10 \%$ and 5\% PTE statistic, in percentage 80

\section{List of Figures}

1 Behaviour of long memory (left) and nonstationary (right) time series. . . . . . 3

2 Autocorrelation functions of $A R(1), M A(1), \operatorname{ARMA}(1,1)$, and $\operatorname{ARMA}(2,2)$. . . 9

3 Data plots of ARIMA $(1,1,1)$, differenced ARIMA $(1,1,1)$, their Sample ACF functions. . . . . . . . . . . . . . . . . . 10 


\section{Symbols and Notation}

\section{Function Spaces}

$L_{2}[a, b]$

$C^{m}[a, b]$

$D[0,1]$ the space of square integrable functions on $[a, b]$

the space of $m$-times continuously differentiable functions on $[a, b]$

the space of real functions on the interval $[0,1]$ that are right continuous and have left-hand limits:

(i) For $0 \leq t \leq 1, x(t+)=\lim _{s \downarrow t} x(s)$ exists and $x(t+)=x(t)$.

(ii) For $0<t \leq 1, x(t-)=\lim _{s \uparrow t} x(s)$ exists.

\section{Convergence}

$f=o(g)$

$f=O(g)$

$o_{P}(\cdot)$

$O_{P}(\cdot)$

"small oh" symbol means that $f(x) / g(x) \rightarrow 0$ as $x \rightarrow a$

"big oh" symbol means that there exists a constant $C>0$ such that $0<|f(x) / g(x)|<C$ as $x \rightarrow a$

"small oh-P" symbol: for two sequences of random variables $\left\{X_{n}\right\}$ and $\left\{Y_{n}\right\}$ we write $X_{n}=o_{P}\left(Y_{n}\right)$ if for any $\varepsilon>0, \mathbf{P}\left(\left|X_{n} / Y_{n}\right|>\varepsilon\right) \rightarrow 0$ as $n \rightarrow \infty$

"big oh-P" symbol: for two sequences of random variables $\left\{X_{n}\right\}$ and $\left\{Y_{n}\right\}$ we write $X_{n}=O_{P}\left(Y_{n}\right)$ if for any $\varepsilon>0$ there exists $c>0$ such that $\mathbf{P}\left(\left|X_{n} / Y_{n}\right|>c\right)<\varepsilon$ as $n \rightarrow \infty$

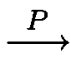

$\stackrel{D}{\longrightarrow}$

$\stackrel{D[0,1]}{\longrightarrow}$

$\stackrel{d}{=}$

$\stackrel{\text { a.s. }}{=}$ convergence in probability

convergence in distribution

convergence in the Skhorohod space $D[0,1]$

equality of the finite-dimensional distributions

equality almost surely

vii 


\section{Processes}

ss

sssi

$f B m$

$f G n$

f.d.d. convergence

$\mathrm{WN}\left(0, \sigma^{2}\right)$

$\operatorname{ARMA}(p, q)$

$\operatorname{ARIMA}(p, d, q)$

$\operatorname{FARIMA}(p, d, q)$ self-similar process (Section 2.2.1)

stationary self-similar increment process (Section 2.2.1)

fractional Brownian motion (Section 2.2.2)

fractional Gaussian noise (Section 2.2.3)

finite-dimensional distribution convergence of $y_{n}(t)$ to $y_{\infty}(t)$ means that for all $k$ and for all $t_{1}, \ldots, t_{k}$, $\left(y_{n}\left(t_{1}\right), \ldots, y_{n}\left(t_{k}\right)\right) \stackrel{D}{\longrightarrow}\left(y_{\infty}\left(t_{1}\right), \ldots, y_{\infty}\left(t_{k}\right)\right)$

White noise, a sequence of uncorrelated random variables with mean zero and constant variance $\sigma^{2}$

Autoregressive Moving Average process with autoregressive parameter $p$ and moving average parameter $q$ (Section 2.1.1)

Integrated Autoregressive Moving Average process with autoregressive parameter $p$, integration parameter $d$ and moving average parameter $q$ (Section 2.1.3)

Fractionally Integrated Autoregressive Moving Average process - an extension of $\operatorname{ARIMA}(p, d, q)$ process with noninteger integration parameter $d$ (Section 2.2.3) 


\section{Introduction}

\subsection{Notion of Time Series}

By time series we mean a sequence $\left\{X_{t}\right\}_{t \in \mathbb{Z}}$ of random variables. This is a stochastic process with "time" space $\mathbb{Z}$. For each $t \in \mathbb{Z}, X_{t}=X_{t}(\omega)$ is a random variable defined on some probability space $(\Omega, \mathcal{F}, P)$. For any fixed $\omega \in \Omega$, the sequence $\left\{X_{t}(\omega)\right\}$ is a realization of the stochastic processes $\left\{X_{t}\right\}$.

Time series theory is a combination of probabilistic and statistical tools and ideas. The probabilistic part consists of identifying and characterizing probability distributions of sets of associated random variables $X_{t}$. Statistical part involves characterizing probability distributions based on real observations, building a model, and ultimately making sound forecasts.

There are immediate problems in both parts that have to be dealt with. First problem is that the data at hand are usually dependent. Second problem comes from the nature of stochastic processes. To have the full characterization of a stochastic process $X_{1}, X_{2}, \ldots$, we need to know all finite-dimensional distributions of this process. Third problem is that time series is a realization of a stochastic process, i.e., statistically speaking, we have sample of size one at each point in time. In addition, in real life the sample is limited in size. These three problems all together make it impossible to characterize probability distributions of time series. In order to cope with the above mentioned problems, the notion of stationarity was introduced. To simplify matters, certain assumptions about the process are usually made. In this thesis, only the processes for which some parameters remain unchanged with time will be considered. The minimal requirements for (weak) stationarity are: the first two moments are constant, and the covariance function depends on the time difference only. In practice, all finite-dimensional distribution approach is not used. Instead, 
the behaviour of time series is usually studied by considering the first two moments of these distributions. The set of second bivariate moments $\operatorname{Cov}\left(X_{s}, X_{t}\right)$ is used to determine the strength of dependence of the present time series value at time $t$ from previous time series values at any time point $s<t$.

There are many sources of nonstationarity in time series. In order to drive nonstationary time series to stationary ones, a number of effective methods have been suggested. Stationary time series are well studied and well suited for forecasting.

As it was noted, the ultimate goal of time series theory is making sound forecasts. An optimal forecast, with respect to the mean squared error, is known to have the form (see, for example, Brockwell and Davis (1991))

$$
\hat{X}_{n+h}=\mathbf{E}\left(X_{n+h} \mid X_{1}, X_{2}, X_{3}, \ldots, X_{n}\right) .
$$

Relation (1.1) shows that independent identically distributed (i.i.d.) sequences are not suited for forecasting. Short memory sequences make forecasting possible but have limited potential. Long memory time series assume that $\sum_{j=-\infty}^{\infty}\left|\operatorname{Cov}\left(X_{0}, X_{j}\right)\right|=\infty$, which make them very useful in long term forecasting. Informally, this means that no matter how far in the future the forecasted variable is, we will still be able to ground our forecast on values of the sample observations. In addition, increments of long memory time series are often stationary.

\subsection{Statement of the Problem}

In this thesis, we are focused on the problem of detection of long range dependence. As it was noted, the long range dependence is a very useful property of time series. Therefore, detecting the presence of long range dependence is important and, if successful, can improve the quality of forecasts significantly. Among others, there is a 
misspecification problem we will have to face. As an example of misspecification we may refer to the fact that nonstationary "unit root" time series and stationary long memory time series, although having most properties different, may behave very similarly. A misspecification may lead to "overdifferencing" and, ultimately, to inferior and biased forecasts. The phenomenon of misspecification is illustrated by Figure 1 below.
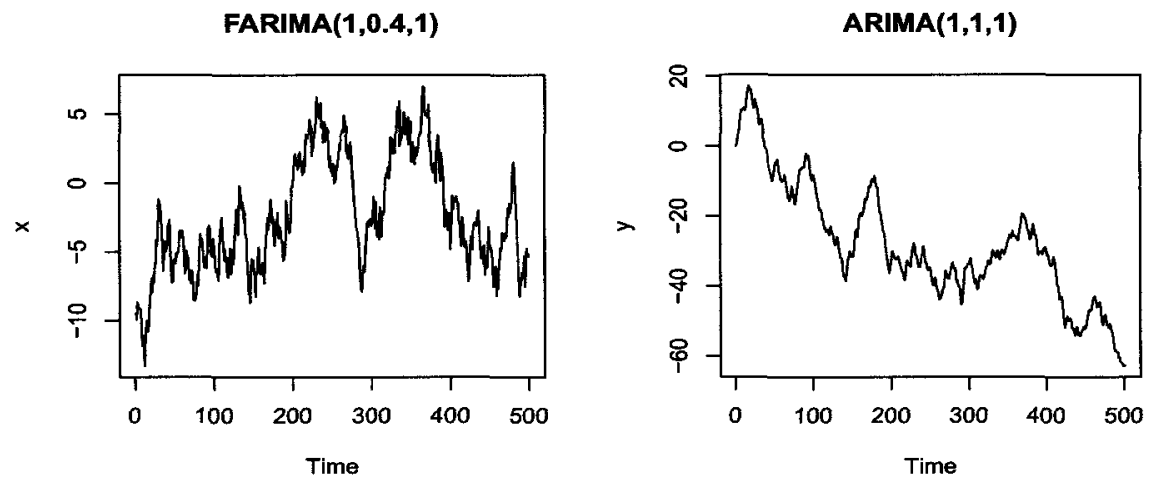

Sample ACF of FARIMA(1,0.4,1)

Sample ACF of ARIMA(1,1,1)
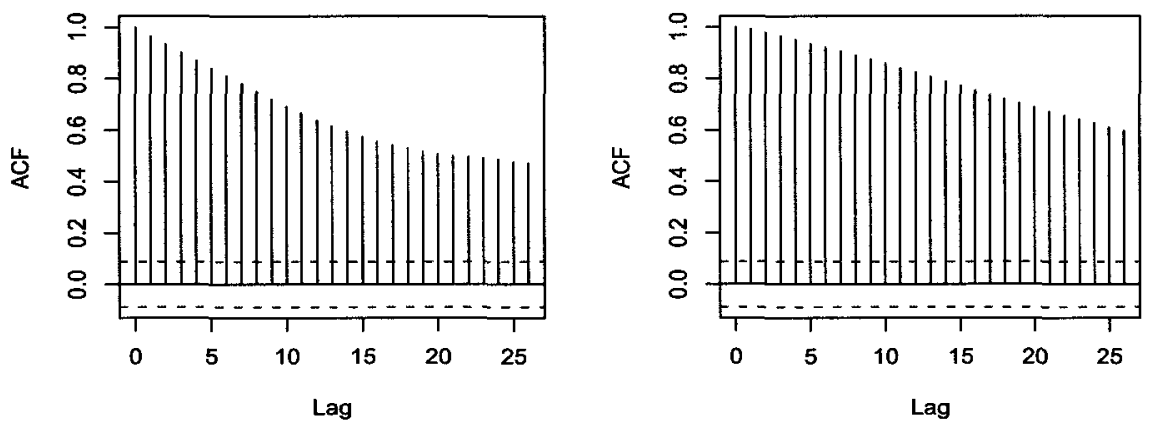

Figure 1: Behaviour of long memory (left) and nonstationary (right) time series.

As seen from Figure 1, in both cases, sample autocorrelation functions die down very slowly. Both data plots show ever changing periodicities and in many other ways look similar. Therefore, visual methods of identification don't work here; more complicated techniques are required. 
In this thesis, we shall address the problem of misspecification by identifying long memory using both estimation and testing techniques.

The thesis is organized as follows. Chapter 2 provides a basic introduction to the topic of long memory time series and gives some classical concepts and results of probability and statistics used in the thesis. To make the material easier to read, the notion of time series is discussed, a review of short memory models is given, and some popular long memory models are considered. Chapter 2 ends with a discussion of some useful convergence concepts. Chapters 3 and 4 contain a detailed discussion of various estimation methods and testing procedures suitable for detecting the presence of long range dependence. Proofs of the results that we use in our work are carefully reviewed, difficult parts of the proofs and omitted details are reconstructed. At the end of Chapter 4, an attempt to improve one of the existing test statistics is made. This is done by adjusting the preliminary test estimation method to the long memory detection problem. In Chapter 5, comparisons of different test statistics are made. For this, the criterion based on the maximal total error probability is used. The estimation and testing methods discussed in the thesis are supplemented with a simulation study. The $\mathrm{R}$ codes used to run simulations are collected in the Appendix. The last chapter is a conclusion. 


\section{Preliminary Information}

To make this thesis self-contained, we begin with a brief overview of stationarity and short memory time series models. Then, we shall motivate the study of long memory processes, and discuss some of the existing long memory models. In conclusion of the section, some important convergence concepts used in the thesis will be discussed.

\subsection{Short Memory Models and Their Properties}

Let us start with the notion of stationarity. Recall that the main feature of weak stationarity consists of having the first two moments constant and autocovariance function depending only on the time lag, $t-s$, that is,

$$
\operatorname{Cov}\left(X_{s}, X_{t}\right)=\gamma_{t-s}=\gamma_{h}
$$

where $h$ is a time difference, which is called lag, and $\gamma_{h}$ denotes the covariance at lag $h$.

Autocovariance function, normalized by the variance, gives the autocorrelation function, called ACF and denoted by $\rho$ :

$$
\rho_{h}=\frac{\gamma_{h}}{\gamma_{0}}
$$

The autocovariance and autocorrelation function possess the following important properties:

1. $\gamma_{0}=\operatorname{Var}\left(X_{t}\right) ; \rho_{0}=1$,

2. $\left|\gamma_{h}\right| \leq \gamma_{0} ;|\rho| \leq 1$,

3. $\gamma_{h}=\gamma_{-h} ; \rho_{h}=\rho_{-h}$, 
4. the autocovariance function is positive semi-definite, that is, for all $n \geq 1$, all integers $t_{1} \ldots t_{n}$, and all real numbers $\alpha_{1} \ldots \alpha_{n}$, one has $\sum_{i=1}^{n} \sum_{j=1}^{n} \alpha_{i} \alpha_{j} \gamma_{\left|t_{2}-t_{j}\right|} \geq 0$. Conversely, every positive semi-definite function $\gamma(s, t)$ is a covariance function of a stochastic process.

The partial autocorrelation function (PACF) defined by $\operatorname{Cor}\left(X_{t}, X_{t+h} \mid X_{t+1}, \ldots, X_{t+h-1}\right)$, gives the understanding of how $X_{t+h}$ depends on $X_{t}$, while taking away the influence of intermediate values for $t+h-1, t+h-2, \ldots, t+1$. Estimates of ACF and PACF play a very important role in the tentative model identification.

\subsubsection{ARMA Model}

Short range dependence is usually defined in terms of covariances $\gamma_{h}=\operatorname{Cov}\left(X_{t}, X_{t+h}\right)$ that converge to zero very fast as $h \rightarrow \infty$. For autoregressive moving average (ARMA) models, the convergence rate is exponential. Therefore the sum of covariances is finite:

$$
\sum_{h=-\infty}^{\infty}\left|\gamma_{h}\right|<\infty
$$

An ARMA model is a stationary model, which is usually referred to as a standard form of short range dependence. In this section, we give a short overview of the ARMA basic structure and properties.

Definition 2.1. (Brockwell and Davis (1991).) The process $\left\{X_{t}, t \in \mathbb{Z}\right\}$ is said to be an $A R M A(p, q)$ process, if $\left\{X_{t}\right\}$ is stationary and for every $t$,

$$
X_{t}-\phi_{1} X_{t-1}-\ldots-\phi_{p} X_{t-p}=\varepsilon_{t}+\theta_{1} \varepsilon_{t-1}+\ldots \theta_{q} \varepsilon_{t-q}
$$

where $\left\{\varepsilon_{t}\right\} \sim W N\left(0, \sigma^{2}\right)$ are called innovations. We say that $\left\{X_{t}\right\}$ is an $\operatorname{ARMA}(p, q)$ process with mean $\mu$ if $\left\{X_{t}-\mu\right\}$ is an $\operatorname{ARMA}(p, q)$ process. 
In a compact form, ARMA model can be written as

$$
\phi(B) X_{t}=\theta(B) \varepsilon_{t}
$$

where for $z \in \mathbb{C}$

$$
\phi(z)=1-\phi_{1} z-\ldots-\phi_{p} z^{p}
$$

and

$$
\theta(z)=1+\theta_{1} z+\ldots+\theta_{q} z^{q}
$$

are the corresponding autoregressive and moving average polynomials. The letter $B$ is used for the backward shift operator $B^{j} X_{t}=X_{t-j}, j=0, \pm 1, \pm 2, \ldots$

$$
\text { If } \phi(z)=1, \text { then }
$$

$$
X_{t}=\theta(B) \varepsilon_{t}
$$

and the process is said to be moving average process of the order $q$, or $\operatorname{MA}(q)$. If $\theta(z)=1$, then the resulting process is

$$
\phi(B) X_{t}=\varepsilon_{t}
$$

and it is called autoregressive process of order $p$, or $\operatorname{AR}(p)$. 


\subsubsection{Causality and Invertibility}

In order to be suitable for forecasting, a sequence $\left\{X_{t}\right\}$ must satisfy three major requirements: causality, invertibility, and stationarity. We have already discussed the notion of stationarity. Let us now define the causality and invertibility for the ARMA models.

Definition 2.2. (Brockwell and Davis (1991).) An ARMA(p,q) time serres defined by the equations $\phi(B) X_{t}=\theta(B) \varepsilon_{t}$ is said to be causal, if there exısts a sequence of constants $\left\{\psi_{\jmath}\right\}$ such that $\sum_{j=0}^{\infty}\left|\psi_{\jmath}\right|<\infty$ and

$$
X_{t}=\sum_{\jmath=0}^{\infty} \psi_{\jmath} \varepsilon_{t-\jmath}, \quad t=0, \pm 1, \pm 2 \ldots
$$

Informally, the definition says that a time series is causal if it depends on the values of the past innovations only.

Another requirement is invertibllity.

Definition 2.3. (Brockwell and Davis (1991).) An ARMA(p,q) process defined by the equations $\phi(B) X_{t}=\theta(B) \varepsilon_{t}$ is said to be invertıble, if there exists a sequence of constants $\left\{\pi_{\jmath}\right\}$ such that $\sum_{j=0}^{\infty}\left|\pi_{\jmath}\right|<\infty$ and

$$
\varepsilon_{t}=\sum_{\jmath=0}^{\infty} \pi_{\jmath} X_{t-\jmath}, \quad t=0, \pm 1, \pm 2 \ldots
$$

As seen from Table 1 below, the causality and invertibility properties show duality between the $\operatorname{AR}(p)$ and $\operatorname{MA}(q)$ representations. 
Table 1: Conditions for stationarity, invertibility, and causality in ARMA models.

\begin{tabular}{||c|c|c|c||}
\hline & stationary & invertible & causal \\
\hline \hline $\operatorname{AR}(p)$ & $\phi(z) \neq 0$, for $|z|=1$ & always & $\phi(z) \neq 0$, for $|z|<1$ \\
\hline $\operatorname{MA}(q)$ & always & $\theta(z) \neq 0$, for $|z|<1$ & always \\
\hline $\operatorname{ARMA}(p, q)$ & $\phi(z) \neq 0$, for $|z|=1$ & $\theta(z) \neq 0$, for $|z|<1$ & $\phi(z) \neq 0$, for $|z|<1$ \\
\hline
\end{tabular}

Note that any non-causal or non-invertible time series can be represented in a causal and invertible form. For details, see Brockwell and Davis (1991), Remark 5 on p. 88 and Proposition 3.5.1 on p. 105.

Without going into technical details, we illustrate graphically fast convergence of ACF to zero for some short range dependence models.
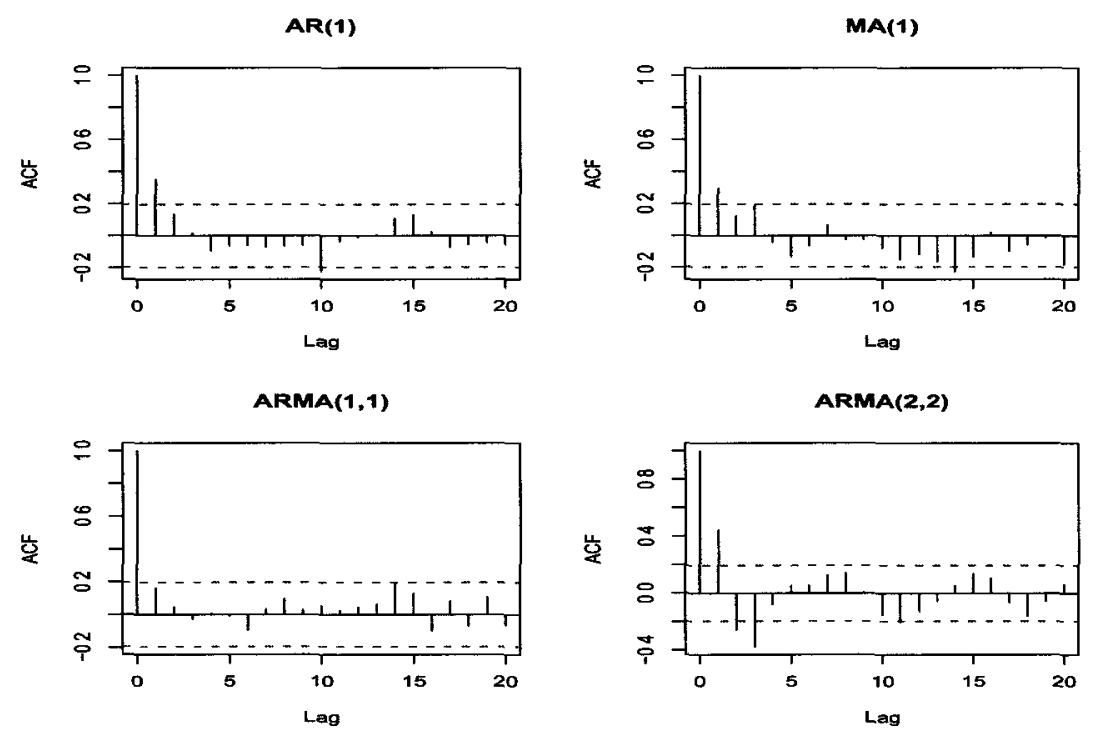

Figure 2: Autocorrelation functions of $A R(1), M A(1), A R M A(1,1)$, and $A R M A(2,2)$. 


\subsubsection{ARIMA Model}

The class of ARIMA models generalizes that of ARMA models and incorporates a wide range of non-stationary time series, which are reduced to ARMA models by differencing finite number of times.

Definition 2.4. (Brockwell and Davis (1991).) If $d$ is a non-negative integer, then $\left\{X_{t}\right\}$ is said to be an $A R I M A(p, d, q)$, if the integrated process $Y_{t}=(1-B)^{d} X_{t}$ is a causal $A R M A(p, q)$ process.

For positive integer $d$, there are several properties related to autocovariance and autocorrelation function that are worth mentioning. Among them,

(a) the covariances $\operatorname{Cov}\left(X_{t}, X_{t+h}\right)$ are time dependent,

(b) $\operatorname{Var}\left(X_{t}\right) \rightarrow \infty$ as $t \rightarrow \infty$.

Consequently, for such series, the sample autocorrelation function fade very slowly. This is illustrated by Figure 3 .
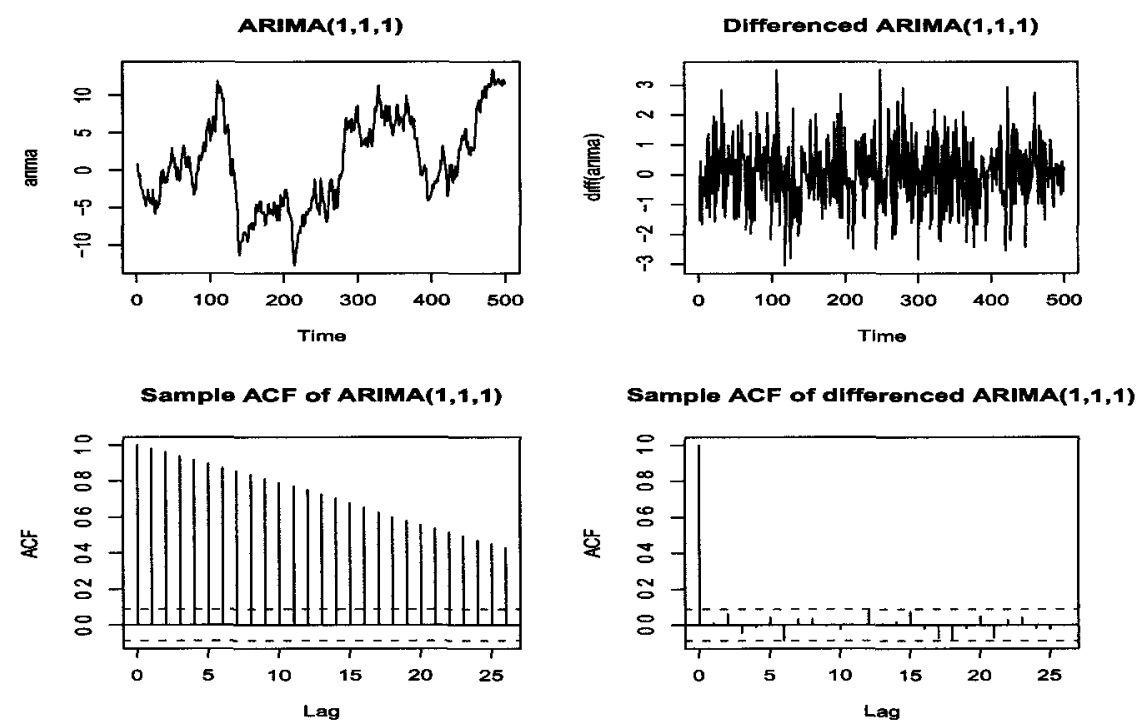

Figure 3: Data plots of ARIMA $(1,1,1)$, differenced ARIMA $(1,1,1)$, their Sample ACF functions. 
The parameter $d$ is called the integration parameter. Further generalization of ARMA models comes when $d$ is not a non-negative integer, but lies in the interval $(-1 / 2,1 / 2)$. Then $\operatorname{ARIMA}(p, d, q)$ becomes fractionally integrated. Fractionally integrated ARMA model, written $\operatorname{FARIMA}(p, d, q)$, has a long memory property for $d \in(0,1 / 2)$. It will be discussed in the next section. 


\subsection{Motivation of Long Memory and Models}

\subsubsection{Self-Similarity}

Definition 2.5. A real-valued stochastic process $Z=\{Z(t)\}_{t \in \mathbb{R}}$ is self-similar with index $H>0$, if for any $a>0$

$$
\{Z(a t)\}_{t \in \mathbb{R}} \stackrel{d}{=}\left\{a^{H} Z(t)\right\}_{t \in \mathbb{R}}
$$

Often, the index $H$ is referred to as a scaling exponent of the process. The term "self-similarity" is usually used in reference to such process since it is invariant in distribution. For convenience, we call self-similar processes with the scaling index $H$ as $H$-ss processes.

For $t>0$, an $H$-ss process $Z(t)$ cannot be stationary unless $Z(t) \equiv 0$. Indeed, if we assume that such a process $Z(t)$ exists, then there would exist $t \in \mathbb{R}$ such that $Z(t) \neq 0$ on an event of positive probability. Then, assuming self-similarity $Z(a t) \stackrel{d}{=} a^{H} Z(t)$ and using the assumed stationarity $Z(t) \stackrel{d}{=} Z(a t)$, we get

$$
\mathbf{E}\left(Z^{2}(t)\right)=a^{2 H} \mathbf{E}\left(Z^{2}(t)\right)
$$

For $a \neq 1$ this is only possible if $\mathbf{E}\left(Z^{2}(t)\right)=0$, which implies $Z(t) \equiv 0$. We arrive at the contradiction.

Although $H$-ss processes cannot be stationary, they can have stationary increments. Let us define precisely the stationarity of increments.

Definition 2.6. A real-valued process $\{Z(t)\}_{t \in \mathbb{R}}$ has stationary increments, if for any $h \in \mathbb{R}$,

$$
\{Z(t+h)-Z(h)\}_{t \in \mathbb{R}} \stackrel{d}{=}\{Z(t)-Z(0)\}_{t \in \mathbb{R}}
$$


A process will be called $H$ self-similar with stationary increments (in short $H$ sssi) if it is self-similar with a scaling index $H$ and has stationary increments (see for example Taqqu in [10]). The class of $H$-sssi processes is very important and will be considered in the thesis in detail. The importance of $H$-sssi processes is seen from the following definition and properties.

Definition 2.7. An incremental H-sssi process $X(k)$ with increments $h=1$ is defined by $X(k)=Z(k+h)-Z(k)=Z(k+1)-Z(k)$ for $k \in \mathbb{Z}$, and constitutes a stationary time series.

The $H$-sssi processes share some common properties:

1. $Z(0) \stackrel{\text { a.s. }}{=} 0$.

This fact is easily seen by taking $t=0$ and noting that $Z(0)=Z(a \cdot 0) \stackrel{d}{=} a^{H} Z(0)$, for $\forall a>0$. For $a>0$ and $H \neq 0$ this can only be possible if $Z(0)=0$.

2. For $H \neq 1, \mathbf{E} Z(t)=0, \forall t \in R$.

This is true because by self-similarity

$$
\mathbf{E} Z(2 t)=2^{H} \mathbf{E} Z(t)
$$

and by the stationarity of increments

$$
\begin{aligned}
\mathbf{E} Z(2 t) & =\mathbf{E}[Z(2 t)-Z(t)+Z(t)]=\mathbf{E}[Z(t+t)-Z(t)]+\mathbf{E} Z(t) \\
& =\mathbf{E}[Z(t)-Z(0)]+\mathbf{E} Z(t)=2 \mathbf{E} Z(t) .
\end{aligned}
$$

Therefore, $2 \mathbf{E} Z(t)=\mathbf{E} Z(2 t)=2^{H} \mathbf{E} Z(t)$. Since we assume $H \neq 1$, this can only be possible if $\mathbf{E} Z(t)=0$.

Property 1 and the stationarity of increments imply the symmetry about zero: 
3. $Z(-t)=Z(-t)-Z(0)=Z(0)-Z(t)=Z(t), \quad \forall t \in \mathbb{R}$.

4. $\operatorname{Var}[Z(t)]=|t|^{2 H} \sigma^{2}$, where $\sigma^{2}=\mathbf{E} Z^{2}(1)$.

Indeed, by Property 3 we have $Z^{2}(t)=Z^{2}(-t)$ and, taking into account self-similarity,

$$
\begin{aligned}
\mathbf{E} Z^{2}(t) & =\mathbf{E} Z^{2}(|t| \cdot \operatorname{sgn} t)=|t|^{2 H} \mathbf{E} Z^{2}(\operatorname{sgn} t) \\
& =|t|^{2 H} \mathbf{E} Z^{2}(1)=|t|^{2 H} \sigma^{2} .
\end{aligned}
$$

5. For $H \neq 1, \mathbf{E} Z(t)=0$ and

$$
\Gamma_{H}(s, t)=\operatorname{Cov}[Z(s), Z(t)]=\frac{\sigma^{2}}{2}\left[|t|^{2 H}+|s|^{2 H}-|t-s|^{2 H}\right]
$$

Indeed, by the stationarity of increments $Z(t)-Z(s) \stackrel{d}{=} Z(t-s)-Z(0)$. Therefore

$$
\begin{aligned}
\Gamma_{H}(s, t) & =\mathbf{E} Z(s) Z(t)=\frac{1}{2}\left[\mathbf{E} Z^{2}(t)+\mathbf{E} Z^{2}(s)-\mathbf{E}(Z(t)-Z(s))^{2}\right] \\
& =\frac{1}{2}\left[\mathbf{E} Z^{2}(t)+\mathbf{E} Z^{2}(s)-\mathbf{E} Z^{2}(t-s)\right]=\frac{\sigma^{2}}{2}\left[|t|^{2 H}+|s|^{2 H}-|t-s|^{2 H}\right] .
\end{aligned}
$$

6. The scaling index $H$ is always less than or equal to 1 .

A short explanation of this property is as follows. We have

$$
\begin{aligned}
\mathbf{E}|Z(2)| & =\mathbf{E}|Z(2)-Z(1)+Z(1)| \leq \mathbf{E}|Z(2)-Z(1)|+\mathbf{E}|Z(1)| \\
& =\mathbf{E}|Z(1)-Z(0)|+\mathbf{E}|Z(1)|=2 \mathbf{E}|Z(1)| .
\end{aligned}
$$

Since $\mathbf{E}|Z(2)|=2^{H} \mathbf{E}|Z(1)|$, it follows that $2^{H} \leq 2$. Thus $H \leq 1$.

The existence of $H$-sssi processes follows from the fact that for $H \in(0,1]$ the covariance function $\Gamma_{H}(s, t)$ is always non-negative. For properties of the covariance 
function, see Section 2.1. For detailed proof, see the paper of M.Taqqu, pp. 8-9, published in [10].

\subsubsection{Fractional Brownian Motion}

Definition 2.8. For a constant parameter $H \in(0,1]$, a Gaussian $H$-sssi process $\left\{W_{H}(t)\right\}_{t \in R}$ with properties 1-6 listed in Section 2.2.1 is called a fractional Brownian motion $(f B m)$. It is called a standard $f B m$ if $\sigma^{2}=\operatorname{Var}\left[W_{H}(1)\right]=1$.

The process $W_{\frac{1}{2}}(t)$ is a standard Brownian motion. The latter is known to have independent increments. Therefore for $H=1 / 2$ the process $W_{H}(t)$ has independent increments.

However, for $H \neq 1 / 2$ the increments of $W_{H}(t)$ are not independent. To see this, consider the autocovariance function of the increment process:

$\gamma_{H}(n)=\operatorname{Cov}\left[W_{H}(t+h)-W_{H}(t), W_{H}(s+h)-W_{H}(s)\right], \quad s+h \leq t, t-s=n h$.

When $n \geq 1$ we have for $h \neq 0$

$$
\begin{aligned}
\gamma_{H}(n)= & \operatorname{Cov}\left(W_{H}(t+h), W_{H}(s+h)\right)-\operatorname{Cov}\left(W_{H}(t), W_{H}(s+h)\right) \\
& +\operatorname{Cov}\left(W_{H}(t+h), W_{H}(s)\right)+\operatorname{Cov}\left(W_{H}(t), W_{H}(s)\right) \\
= & \frac{\sigma^{2}}{2}\left(-2|t-s|^{2 H}+|t-s-h|^{2 H}+|t-s+h|^{2 H}\right) \\
= & \frac{\sigma^{2}}{2}\left(-2|n h|^{2 H}+|n h-h|^{2 H}+|n h+h|^{2 H}\right) \\
= & \frac{|h|^{2 H} \sigma^{2}}{2}\left(-2 n^{2 H}+(n-1)^{2 H}+(n+1)^{2 H}\right) .
\end{aligned}
$$

Lemma 2.1. Let the autocovariance function $\gamma_{H}(n)$ of incremental process of $f B m$ be defined by (2.2). Then for $n \neq 0$ 
(i) $\gamma_{H}(n)<0$ for $0<H<1 / 2$,

(ii) $\gamma_{H}(n)=0$ for $H=1 / 2$,

(iii) $\gamma_{H}(n)>0$ for $1 / 2<H<1$.

Proof. Note that for $0<H<1 / 2$ the function $f(t)=t^{2 H}$, where $t=\{n-1, n, n+1\}$, is strictly concave. Similarly, for $1 / 2<H<1, f(t)$ is a strictly convex. Therefore for $0<H<1 / 2$

$$
\begin{aligned}
(n-1)^{2 H}+(n+1)^{2 H} & =f(n-1)+f(n+1) \\
& <f(n-1+n+1)=f(2 n)=2 n^{2 H}
\end{aligned}
$$

and

$\gamma_{H}(n)=\frac{|h|^{2 H} \sigma^{2}}{2}\left(-2 n^{2 H}+(n-1)^{2 H}+(n+1)^{2 H}\right)<\frac{|h|^{2 H} \sigma^{2}}{2}\left(-2 n^{2 H}+2 n^{2 H}\right)<0$

In a similar way, using convexity, we can show that for $1 / 2<H<1, \gamma_{H}(n)>0$.

It is seen from Lemma 2.1 that depending on whether $0<H<1 / 2, H=1 / 2$, or $1 / 2<H<1$, the behavior of $f B m$ can be very different. For $1 / 2<H<1$, the increments of $\mathrm{fBm}$ are positively correlated, and the process exhibits aggregation behavior. Therefore it is well suited for modeling persistence and memory processes. On the contrary, for $0<H<1 / 2$, the increments of $f B m$ are negatively correlated, and thus the process could be used to model intermittent and antipersistent processes. For $H=1 / 2$, a $f B m$ becomes a regular Brownian motion with independent increments. The Brownian motion has been extensively studied, and its applications are well known. 


\subsubsection{Definition of Long Memory}

Definition 2.9. We say that a finite-variance stationary stochastic process $\left\{X_{t}\right\}$ has long memory if its autocovariance function is of the form $\gamma(k) \sim c k^{2 d-1}$, where $c>0$ and $d \in(0,1 / 2)$.

For a long memory process $\left\{X_{t}\right\}$ with autocovariance function $\gamma(k)$ it is true that

$$
\lim _{k \rightarrow \infty} \frac{\gamma(k)}{c k^{2 d-1}}=1 \quad \text { and } \quad \sum_{k=-\infty}^{\infty} \gamma(k)=\infty
$$

Referring back to Section 2.2.2, note that long memory does not necessarily imply positive covariances at all lags. Often, in long memory time series, constant $c$ in the autocovariance function $\gamma(k)$ is substituted by a slowly varying function. By the slowly varying function we mean a function $L(n)$ such that for any $a>0$, $L($ an $) / L(n) \rightarrow 1$ as $n \rightarrow \infty$. For instance, the function $L(n)=\log n$ is slowly varying.

It follows from the definition that for $1 / 2<H<1$, taking $d=H-1 / 2$, the increments of $f B m$ exhibit long memory, since as $k \rightarrow \infty$

$$
\gamma_{H}(k)=\frac{|h|^{2 H} \sigma^{2}}{2}\left(-2 k^{2 H}+(k-1)^{2 H}+(k+1)^{2 H}\right) \sim c_{H} k^{2 H-2}
$$

where

$$
c_{H}=|h|^{2 H} \sigma^{2} H(2 H-1) .
$$

This can be checked by making the transformation

$$
\begin{aligned}
\gamma_{H}(k) & =\frac{|h|^{2 H} \sigma^{2}}{2}\left(-2 k^{2 H}+(k-1)^{2 H}+(k+1)^{2 H}\right) \\
& =\gamma_{H}(k)=\frac{|h|^{2 H} \sigma^{2}}{2} k^{2 H-2}\left(k^{2}\left[\left(1-\frac{1}{k}\right)^{2 H}-2+\left(1+\frac{1}{k}\right)^{2 H}\right]\right)
\end{aligned}
$$


and noting that as $k \rightarrow \infty$

$$
k^{2}\left[\left(1-\frac{1}{k}\right)^{2 H}-2+\left(1+\frac{1}{k}\right)^{2 H}\right] \rightarrow 2 H(2 H-1) .
$$

Relation (2.4) is obtained by using the second order Taylor expansion:

$$
\begin{aligned}
& k^{2}\left[\left(1-\frac{1}{k}\right)^{2 H}-2+\left(1+\frac{1}{k}\right)^{2 H}\right] \approx k^{2}\left[1-\frac{2 H}{k}+\frac{2 H(2 H-1)}{2 k^{2}}\right]+ \\
& k^{2}\left[-2+1+\frac{2 H}{k}+\frac{2 H(2 H-1)}{2 k^{2}}\right]=k^{2}\left[\frac{2 H(2 H-1)}{k^{2}}\right]=2 H(2 H-1) .
\end{aligned}
$$

Long memory was empirically discovered by Harold E. Hurst in 1951. To signify this discovery, the parameter $H$, which determines the long memory property, was named the Hurst exponent.

\subsubsection{Fractional Gaussian Noise}

Recall our description of an $H$-sssi process $Z(t)_{t \in \mathbb{R}}$ and notice the similarity with the $f B m W_{H}(t)$. According to Definition 2.7, the increment process of $f B m$ for increments $h=1$, namely $W_{H}(k+1)-W_{H}(k), k \in \mathbb{Z}$, is a time series.

Definition 2.10. If $\left\{W_{H}(t)\right\}_{t \in \mathbb{R}}$ is a fBm, then the process $\left\{X_{k}\right\}_{k \in \mathbb{Z}}$ defined by $X_{k}=$ $W_{H}(k+1)-W_{H}(k)$ is called a fractional Gaussian noise ( $\left.f G n\right)$.

For $1 / 2<H<1$ the fractional Gaussian noise process $(f G n)$ is a simple and popular representation of time series, having a long memory property. Its autocovariance function asymptotically, as $k \rightarrow \infty$, satisfies

$$
\gamma_{H}(k) \sim \sigma^{2} H(H-1) k^{2 H-2}, \quad \text { where } k \text { is the lag. }
$$

Although $f G n$ exhibits long memory for $1 / 2<H<1$, its autocovariance function is 
specified at all lags, as one can see from equality (2.3). Therefore, $f G n$ could be inappropriate for modeling certain data with long memory dependence. To accommodate for different covariances at short lags, another model, called $\operatorname{FARIMA}(p, d, q)$, is used.

\subsubsection{FARIMA Model}

In this subsection, we discuss the $\operatorname{FARIMA}(p, d, q)$ model, which is an extension of the short memory stationary time series $\operatorname{ARMA}(p, q)$ model.

Definition 2.11. For $|d|<1 / 2$, a process $\left\{Y_{t}\right\}$ is called a FARIMA $(p, d, q)$ process with mean $\mu$, if $X_{t}=Y_{t}-\mu$ satisfies

$$
\phi(B)(1-B)^{d} X_{t}=\theta(B) \varepsilon_{t}
$$

where $\left\{\varepsilon_{t}\right\}$ is a white noise process with zero mean and constant variance $\sigma_{\varepsilon}^{2}$, and

$$
\begin{aligned}
& \phi(B)=1-\phi_{1} B-\phi_{2} B^{2}-\ldots-\phi_{p} B^{p}, \\
& \theta(B)=1+\theta_{1} B+\theta_{2} B^{2}+\ldots+\theta_{q} B^{q}
\end{aligned}
$$

are polynomials of order $p$ and $q$ having no common roots, with roots lying outside of the unit circle. $B$ is a backward shift operator such that $B^{i} X_{t}=X_{t-i}$ and $B^{j} \varepsilon_{t}=\varepsilon_{t-j}$, for all $i, j \in \mathbb{Z}$.

If the process $\left\{X_{t}\right\}$ is invertible and causal, we can write

$$
X_{t}=\phi^{-1}(B) \theta(B)(1-B)^{-d} \varepsilon_{t} .
$$

The above introduced $\operatorname{FARIMA}(p, d, q)$ model has three parameters. The parameters $p$ and $q$ take care of short range dependence, whereas $d$ is a long memory 
parameter. Let us consider the parameter $d$ more closely. For simplicity, we assume that there is no short range dependence in equality (2.5). Then

$$
(1-B)^{d} X_{t}=\varepsilon_{t}, \quad \varepsilon_{t} \sim W N\left(0, \sigma_{\varepsilon}^{2}\right)
$$

If $\left\{X_{t}\right\}$ is stationary, we can rewrite $(2.6)$ as

$$
X_{t}=(1-B)^{-d} \varepsilon_{t}
$$

where

$$
(1-B)^{-d} \varepsilon_{t}=\sum_{\jmath=0}^{\infty}\left(\begin{array}{c}
-d \\
j
\end{array}\right)(-B)^{\jmath} \varepsilon_{t}=\sum_{\jmath=0}^{\infty} \psi_{\jmath} \varepsilon_{t-\jmath}
$$

and

$$
\begin{aligned}
\psi_{\jmath} & =\left(\begin{array}{c}
-d \\
j
\end{array}\right)(-1)^{\jmath}=\frac{(-d)(-d-1) \ldots(-d-j+1)}{j !}(-1)^{\jmath} \\
& =\frac{(j+d-1)(j+d-2) \ldots(j+d-j)}{j !}=\frac{\Gamma(j+d)}{\Gamma(j+1) \Gamma(d)}
\end{aligned}
$$

Here $\Gamma(x)=\int_{0}^{\infty} t^{x-1} e^{-t} d t, x>0$, is the Gamma function.

It is well known that as $x \rightarrow \infty$

$$
\Gamma(x) \sim \sqrt{2 \pi} e^{-x} x^{x-1 / 2}
$$

The relation $f(x) \sim g(x)$ means that $\lim _{x \rightarrow \infty} f(x) / g(x)=1$. Consequently,

$$
\psi_{\jmath}=\frac{\Gamma(j+d)}{\Gamma(j+1) \Gamma(d)} \sim \frac{1}{\Gamma(d)} \frac{\sqrt{2 \pi} e^{-\jmath-d}(j+d)^{\jmath+d-1 / 2}}{\sqrt{2 \pi} e^{-\jmath-1}(j+1)^{\jmath+1-1 / 2}} \sim \frac{1}{\Gamma(d) j^{1-d}}
$$


and the sequence $\left\{\psi_{\jmath}\right\}$ in (2.7) is square summable if and only if $2(1-d)>1$. From here we have that $d<1 / 2$. Similarly, we can show that $\left\{X_{t}\right\}$ is invertible for $d>$ $-1 / 2$. Therefore for $|d|<1 / 2, \operatorname{FARIMA}(p, d, q)$ is stationary and invertible. Relating back to the Hurst exponent, we get the relationship $H=d+1 / 2$, or equivalently, $d=H-1 / 2$.

\subsubsection{Spectral Density of FARIMA}

In time series analysis, the use of autocovariances to study the dependence structure and evolution of stochastic processes, is called the tıme domain approach. Up to this section, the time domain approach in the analysis has been used. However, there is an alternative one, called frequency domain approach. It is based on the fact that any stationary process $\left\{X_{t}\right\}$ can be decomposed into different frequency components as

$$
X_{t}=\sum_{\jmath=1}^{n} A\left(\lambda_{\jmath}\right) e^{\imath t \lambda}
$$

where $-\pi<\lambda_{3} \leq \pi$ and $\left\{A\left(\lambda_{3}\right)\right\}$ is a sequence of uncorrelated zero mean random coefficients with a common variance $\sigma_{A}^{2}$. Then, the autocovariance function can be represented as

$$
\gamma_{h}=\int_{(-\pi, \pi]} e^{\imath h \lambda} d F(\lambda)
$$

where $F(\lambda)$ is a spectral distribution function. For details, see Brockwell and Davis (1991), pp. 116-117. In many cases, such as short memory and long memory time series, $F$ will have a density $f$, and

$$
\gamma_{h}=\int_{-\pi}^{\pi} e^{\imath h \lambda} f(\lambda) d \lambda .
$$


The spectral density can also be seen as a Fourier transform of the autocovariance function:

$$
f(\lambda)=\frac{1}{2 \pi} \sum_{k=-\infty}^{\infty} \gamma_{k} e^{-\imath \lambda k}
$$

From (2.8) and (2.9) we can see a duality between the spectral density and autocovariance function: if we know the spectral density, we can derive autocovariance function and vice versa. The frequency domain approach is important because it gives us additional tools for the analysis of time series. For example, if $\gamma_{k}$ are absolutely summable (short-range dependence case), then from (2.9) it will follow that, being a uniform limit of a sequence of continuous functions, $f$ will be continuous. On the contrary, as we will see later, in case of long-range dependence, the spectral density is unbounded at $\lambda=0$. In this section, we will consider the spectral density of $\operatorname{FARIMA}(p, d, q)$ process.

Theorem 2.2. (Brockwell and Davis (1991).) Suppose we have a FARIMA $(p, d, q)$ process with $d \in(-1,1 / 2)$. Assume that the polynomials $\phi(z)$ and $\theta(z)$ have no common roots and their roots lue outside of the unit curcle $\{z:|z| \leq 1\}$. Then there exists a unıque stationary solution, which is invertıble and causal, given by

$$
X_{t}=\sum_{\jmath=-\infty}^{\infty} \psi_{\jmath} \varepsilon_{t-\jmath}
$$

where $\psi(z)=(1-z)^{-d} \theta(z) / \phi(z)$

Taking into account that $\left\{\varepsilon_{t}\right\}$ is a (stationary) sequence of independent random 
variables with $\mathbf{E}\left(\varepsilon_{t}\right)=0$ and $\operatorname{Var}\left(\varepsilon_{t}\right)=\sigma^{2}$, the covariance function of $X_{t}$ is given by

$$
\begin{aligned}
\gamma_{k} & =\mathbf{E}\left(X_{t} X_{t+k}\right)=\mathbf{E}\left(\sum_{\imath=0}^{\infty} \sum_{\jmath=0}^{\infty} \psi_{\imath} \psi_{\jmath} \varepsilon_{t-\imath} \varepsilon_{t+k-\jmath}\right) \\
& =\sum_{\imath=0}^{\infty} \psi_{\imath} \psi_{\imath+k} \mathbf{E}\left(\varepsilon_{t-\imath}^{2}\right)=\sigma^{2} \sum_{\imath=0}^{\infty} \phi_{\imath} \phi_{\imath+k} .
\end{aligned}
$$

The covariance generating function of $X_{t}$ is defined as

$$
\gamma(B)=\sum_{k=-\infty}^{\infty} \gamma_{k} B^{k}
$$

Using (2.11), we can rewrite $\gamma(B)$ from (2.12) as follows:

$$
\begin{aligned}
\gamma(B) & =\sigma^{2} \sum_{k=-\infty}^{\infty} \sum_{\imath=-\infty}^{\infty} \psi_{\imath} \psi_{\imath+k} B^{k}=\sigma^{2} \sum_{k=-\infty}^{\infty} \sum_{\imath=-\infty}^{\infty} \psi_{\imath} \psi_{\jmath} B^{\jmath-\imath} \\
& =\sigma^{2} \sum_{\jmath=0}^{\infty} \psi_{\jmath} B^{\jmath} \sum_{\imath=0}^{\infty} \psi_{\imath} B^{-\imath}=\sigma^{2} \psi(B) \psi\left(B^{-1}\right)
\end{aligned}
$$

Recall the definition of spectral density in (2.9). By comparing the spectral density and covariance generating function formulas one gets the relation

$$
f(\lambda)=\frac{1}{2 \pi} \gamma\left(e^{-\imath \lambda}\right)=\frac{\sigma^{2}}{2 \pi} \psi\left(e^{-\imath \lambda}\right) \psi\left(e^{\imath \lambda}\right)
$$

In the linear representation of $\operatorname{FARIMA}(p, d, q)$ given by $(2.10)$, the coefficients $\psi_{J}$ are defined as $\psi(z)=(1-z)^{-d} \theta(z) / \phi(z)$. Therefore

$$
\begin{aligned}
f(\lambda) & =\frac{1}{2 \pi} \sigma^{2} \frac{\theta\left(e^{-\imath \lambda}\right)\left(1-e^{-\imath \lambda}\right)^{-d}}{\phi\left(e^{-\imath \lambda}\right)} \cdot \frac{\theta\left(e^{\imath \lambda}\right)\left(1-e^{\imath \lambda}\right)^{-d}}{\phi\left(e^{\imath \lambda}\right)} \\
& =\frac{\sigma^{2}}{2 \pi}\left|1-e^{-\imath \lambda}\right|^{-2 d} \frac{\left|\theta\left(e^{-\imath \lambda}\right)\right|^{2}}{\left|\phi\left(e^{-\imath \lambda}\right)\right|^{2}}=\frac{\sigma^{2}}{2 \pi}\left[2 \sin \left(\frac{\lambda}{2}\right)\right]^{-2 d} \frac{\left|\theta\left(e^{-\imath \lambda}\right)\right|^{2}}{\left|\phi\left(e^{-\imath \lambda}\right)\right|^{2}} .
\end{aligned}
$$


Note that

$$
\begin{aligned}
\left|1-e^{-i \lambda}\right|^{-2 d} & =|1-\cos \lambda-i \sin \lambda|^{-2 d}=\left[(1-\cos \lambda)^{2}+\sin ^{2} \lambda\right]^{-d} \\
& =[2(1-\cos \lambda)]^{-d}=\left[4\left(\frac{1-\cos \lambda}{2}\right)\right]^{-d}=\left[2 \sin \left(\frac{\lambda}{2}\right)\right]^{-2 d} .
\end{aligned}
$$

Hence in (2.13) we can use $\left[2 \sin \left(\frac{\lambda}{2}\right)\right]^{-2 d}$ instead of $\left|1-e^{-i \lambda}\right|^{-2 d}$. Next, as $\lambda \rightarrow 0$, we have $\sin (\lambda / 2) \sim \lambda / 2$ and $e^{-i \lambda} \sim 1$. Therefore

$$
f(\lambda) \sim G|\lambda|^{-2 d}, \quad \text { where } \quad G=\frac{\sigma^{2}}{2 \pi} \frac{|\theta(1)|^{2}}{|\phi(1)|^{2}}
$$

which indicates that $f$ is unbounded at $\lambda=0$ when $0<d<1 / 2$ (long memory case).

By Theorem 4.4.2 in Brockwell and Davis (1991), if the sequence $\left\{u_{t}\right\}$ belongs to $\operatorname{ARMA}(p, q)$, then its spectral density is given by

$$
f_{u}(\lambda)=\frac{\sigma^{2}}{2 \pi} \frac{\left|\theta\left(e^{-i \lambda}\right)\right|^{2}}{\left|\phi\left(e^{-i \lambda}\right)\right|^{2}}, \quad-\pi \leq \lambda \leq \pi
$$

Therefore we can also write the spectral density $f_{X}(\lambda)$ of $\operatorname{FARIMA}(p, d, q)$ in the form

$$
f_{X}(\lambda) \approx|\lambda|^{-2 d} f_{u}(\lambda)
$$

where $f_{u}(\lambda)$ is the spectral density of the corresponding $\operatorname{ARMA}(p, q)$ process. It is also common to write the spectral density of $\operatorname{FARIMA}(p, d, q)$ as

$$
f_{X}(\lambda)=\left|1-e^{-i \lambda}\right|^{-2 d} f_{u}(\lambda) \quad \text { or } \quad f_{X}(\lambda)=\left[2 \sin \left(\frac{\lambda}{2}\right)\right]^{-2 d} f_{u}(\lambda)
$$

where $-\pi \leq \lambda \leq \pi$. 


\subsection{Some Important Concepts in Convergence}

\subsubsection{Convergence in $L_{2}[0,1]$}

Convergence concepts will become an important part of the proofs in Chapter 4. In most cases, proofs in the classical papers on partial sum processes have been done in the Skorohod $D[0,1]$ space. In a few cases discussed in this thesis, a weaker $L_{2}$-topology is required. Below we formulate an important theorem for the $L_{2}[0,1]$ convergence followed by a Corollary taken from the article of Giraitis et al. (2006), which is an adaptation of the result of Cremers and Kadelka (1986) for $L_{p}[0,1]$.

Theorem 2.3. Assume that $\left\{X_{n}(t), t \in[0,1]\right\}, n \geq 0$, is a sequence of real-valued measurable processes with paths from the space $L_{2}[0,1]$, i.e.,

$$
\int_{0}^{1} X_{n}^{2}(t) d t<\infty \quad \text { a.s. }
$$

Suppose that there exists a process $X_{\infty}(\cdot)$ and a nonnegative function $f$ satisfying $\int_{0}^{1} f(t) d t<\infty$, and such that

(i) the finite-dimensional distributions of $X_{n}(\cdot)$ converge to those of the process $X_{\infty}(\cdot)$

(ii) for any $t \in[0,1]$ and all $n \geq 1, \mathbf{E} X_{n}^{2}(t) \leq f(t)$;

(iii) for any $t \in[0,1], \mathbf{E} X_{n}^{2}(t) \rightarrow \mathbf{E} X_{\infty}^{2}(t)$ as $n \rightarrow \infty$.

Then $X_{n}$ converges weakly to $X_{\infty}$ in $L_{2}[0,1]$, i.e., for any continuous real-valued bounded functional $g: L_{2}[0,1] \rightarrow \mathbb{R}$, the relation

$$
\mathbf{E} g\left(X_{n}\right) \rightarrow \mathbf{E} g\left(X_{\infty}\right), \quad n \rightarrow \infty
$$


holds. In particular, for any continuous function $h: L_{2}[0,1] \rightarrow L_{2}[0,1], h\left(X_{n}(\cdot)\right)$ converges weakly to $h\left(X_{\infty}(\cdot)\right)$.

As an immediate corollary we get:

Corollary 2.3. Under the conditions of Theorem 2.3, as $n \rightarrow \infty$

$$
h\left(X_{n}(\cdot)\right) \stackrel{D}{\longrightarrow} h\left(X_{\infty}(\cdot)\right)
$$

for any continuous functional $h: L_{2}[0,1] \rightarrow \mathbb{R}$ of paths $X_{n}(\cdot)$.

In particular, the above convergence result applies to the functionals

$$
\begin{aligned}
& \int_{0}^{1} X_{n}^{2}(t) d t, \int_{0}^{1} X_{n}(t) d t, \int_{0}^{1}\left(X_{n}(t)-t X_{n}(1)-\int_{0}^{1}\left(X_{n}(u)-u X_{n}(1)\right) d u\right)^{2} d t \\
& \int_{0}^{1}\left[\int_{0}^{t} X_{n}(s) d s-t \int_{0}^{1} X_{n}(u) d u-\int_{0}^{1}\left(\int_{0}^{v} X_{n}(s) d s-v \int_{0}^{1} X_{n}(u) d u\right) d v\right]^{2} d t .
\end{aligned}
$$

\subsubsection{Donsker Type Theorems}

The following theorem is a combination of various mean convergence results. All those results are available in research articles related to short and long memory processes, and well known to specialists. For details on convergence of short memory sequences, see, for example, Giraitis and Surgailis (1985) and Surgailis (1982). Good references on convergence of long memory sequences are Davydov (1970), Dobrushin and Major (1979), and Taqqu (1975).

Theorem 2.4. Let $X_{\jmath}, j=1,2, \ldots$, be a sequence of random variables, and let $\bar{X}=\bar{X}_{[n t]}=\sum_{\jmath=1}^{[n t]} X_{\jmath}$, where $t \in[0,1], n=1,2, \ldots$, and $[n t]$ is the largest integer less than or equal to $n t$. 


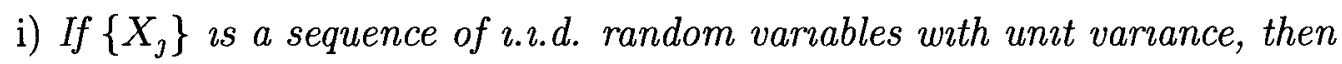

$$
\frac{\sum_{j=1}^{[n t]}\left(X_{\jmath}-\mathbf{E}\left(X_{1}\right)\right)}{\sqrt{n}} \stackrel{D[0,1]}{\longrightarrow} W(t), \quad \text { as } \quad n \rightarrow \infty,
$$

where $W(t)$ is a Brownıan motıon. Also,

$$
\frac{\sum_{\jmath=1}^{[n t]}\left(X_{\jmath}-\bar{X}\right)}{\sqrt{n}} \stackrel{D[0,1]}{\longrightarrow} W^{0}(t), \quad \text { as } \quad n \rightarrow \infty,
$$

where $W^{0}(t)$ is a Brownıan bridge defined by $W^{0}(t)=W(t)-t W(1)$.

ii) If $\left\{X_{j}\right\}$ is the sequence of random variables with short memory, as defined in Section 2.1.1, and belongs to one of the two cases:

(a) $X_{t}=G\left(\varepsilon_{t}\right)$, where $\varepsilon_{t}$ are standard Gaussian,

(b) $X_{t}=\sum_{\jmath=1}^{\infty} a_{\jmath} \varepsilon_{t-\jmath}$, where $\varepsilon_{t}$ are i.l.d. random varıables with $\mathbf{E}\left(\varepsilon_{t}\right)=0$ and $\operatorname{Var}\left(\varepsilon_{t}\right)=1$,

then

$$
\frac{\sum_{j=1}^{[n t]}\left(X_{\jmath}-\mathbf{E}\left(X_{1}\right)\right)}{\sigma \sqrt{n}} \stackrel{f d d}{\longrightarrow} W(t), \quad \text { as } \quad n \rightarrow \infty,
$$

where $W(t)$ is a Brownian motıon, $\sigma^{2}=\sum_{\jmath=-\infty}^{\infty} \gamma_{\jmath}<\infty$, and $\gamma_{\jmath}=\operatorname{Cov}\left(X_{t}, X_{t+\jmath}\right)$. Also,

$$
\frac{\sum_{j=1}^{[n t]}\left(X_{j}-\bar{X}\right)}{\sigma \sqrt{n}} \stackrel{f d d}{\longrightarrow} W^{0}(t), \quad \text { as } \quad n \rightarrow \infty
$$


where $W^{0}(t)$ is a Brownian bridge.

iii) If $\left\{X_{j}\right\}$ is a sequence of random variables with long memory, as defined in Section 2.2.3, and belongs to one of the two cases:

(a) $X_{t}=G\left(\varepsilon_{t}\right)$, where $\varepsilon_{t}$ are standard Gaussian, and $G$ satisfies $\mathbf{E}\left(\varepsilon_{1} G\left(\varepsilon_{1}\right)\right) \neq 0$,

(b) $X_{t}=\sum_{j=1}^{\infty} a_{j} \varepsilon_{t-j}$, where $\varepsilon_{t}$ are i.i.d. random variables with $\mathbf{E}\left(\varepsilon_{t}\right)=0$ and $\operatorname{Var}\left(\varepsilon_{t}\right)=1$,

then

$$
\frac{\sum_{j=1}^{[n t]}\left(X_{j}-\mathbf{E}\left(X_{1}\right)\right)}{c_{d} n^{\frac{1}{2}+d}} \stackrel{D[0,1]}{\longrightarrow} W_{\frac{1}{2}+d}(t), \quad \text { as } \quad n \rightarrow \infty,
$$

where $c_{d}$ is a positive number and $W_{\frac{1}{2}+d}(t)$ is a fractional Brownian motion. Also,

$$
\frac{\sum_{j=1}^{[n t]}\left(X_{j}-\bar{X}\right)}{c_{d} n^{\frac{1}{2}+d}} \stackrel{D[0,1]}{\longrightarrow} W_{\frac{1}{2}+d}^{0}(t), \quad \text { as } \quad n \rightarrow \infty,
$$

where $W_{\frac{1}{2}+d}^{0}(t)$ is a fractional Brownian bridge.

As an illustration, let us show that the substitution of $\mathbf{E}(X)$ by $\bar{X}$ in the short memory case ii) causes change in the limiting process from Brownian motion to Brownian bridge. The long memory case can be analyzed similarly. 
Noting that $[n t] / n \rightarrow t$ as $n \rightarrow \infty$, we get

$$
\begin{aligned}
\sum_{j=1}^{[n t]} \frac{\left(X_{j}-\bar{X}\right)}{\sigma \sqrt{n}} & =\sum_{j=1}^{[n t]} \frac{\left(X_{\jmath}-\mathbf{E}\left(X_{1}\right)+\mathbf{E}\left(X_{1}\right)-\bar{X}\right)}{\sigma \sqrt{n}}=\sum_{j=1}^{[n t]} \frac{X_{\jmath}-\mathbf{E}\left(X_{1}\right)}{\sigma \sqrt{n}} \\
& -\sum_{\jmath=1}^{[n t]} \frac{\bar{X}-\mathbf{E}\left(X_{1}\right)}{\sigma \sqrt{n}}=: W_{n}(t)-\frac{[n t]\left(\sum_{\imath=1}^{n} \frac{X_{2}}{n}-\mathbf{E}\left(X_{1}\right)\right)}{\sigma \sqrt{n}} \\
& =W_{n}(t)-\frac{[n t] \sum_{j=1}^{n}\left(\frac{X_{j}-\mathbf{E}\left(X_{1}\right)}{n}\right)}{\sigma \sqrt{n}}=W_{n}(t)-\frac{[n t]}{n} \sum_{j=1}^{n}\left(X_{\jmath}-\mathbf{E}\left(X_{1}\right)\right) \\
& \stackrel{D}{\longrightarrow} W(t)-t W(1) .
\end{aligned}
$$

\subsubsection{Cumulants}

Among conditions of the theorems stated in Chapter 4, one is concerned with cumulants. The concept of cumulant is very interesting and important, but rarely discussed. We shall briefly touch it here.

Definition 2.12. For a random variable $X$, the cumulant $\kappa_{n}$ of order $n$ of $X$ is the coefficient of $(\imath t)^{n} / n !$ in the Taylor series expansion of the function $\log \left[\mathbf{E}\left(e^{\imath t X}\right)\right]$.

The cumulants of a random variable $X$ are closely related to its moments. Indeed, it follows from the expansion

$$
e^{\imath t X}=1+i t X-\frac{t^{2} X^{2}}{2 !}-\ldots=\sum_{n=0}^{\infty} \frac{(i t X)^{n}}{n !}
$$

that the characteristic function $\mathbf{E}\left(e^{\imath t X}\right)$ of $X$ can be represented as follows:

$$
\mathbf{E}\left(e^{\imath t X}\right)=\sum_{n=0}^{\infty} \frac{m_{n}(i t)^{n}}{n !}, \quad \text { where } \quad m_{n}=\mathbf{E}\left(X^{n}\right)
$$


Using the Taylor series expansion of a logarithm function, we have

$$
\log (X)=\sum_{n=1}^{\infty} \frac{(-1)^{n+1}(X-1)^{n}}{n}=-\sum_{n=1}^{\infty} \frac{1}{n}(1-X)^{n}
$$

Thus,

$$
\begin{gathered}
\log \left[\mathbf{E}\left(e^{i t X}\right)\right]=-\sum_{n=1}^{\infty} \frac{1}{n}\left(1-\mathbf{E}\left(e^{i t X}\right)\right)^{n}=-\sum_{k=1}^{\infty} \frac{1}{k}\left(-\sum_{n=1}^{\infty} \frac{m_{n}(i t)^{n}}{n !}\right)^{k} \\
=m_{1} i t-\left(m_{2}-m_{1}^{2}\right) \frac{t^{2}}{2 !}-\left(m_{3}-3 m_{2} m_{1}+2 m_{1}^{3}\right) \frac{i t^{3}}{3 !}+\ldots=\sum_{n=1}^{\infty} \kappa_{n} \frac{(i t)^{n}}{n !} .
\end{gathered}
$$

For example, $\kappa_{1}=\mathbf{E}(X)$ and $\kappa_{2}=\operatorname{Var}(X)$.

For a standard normal random variable, the characteristic function is $\phi(t)=e^{-\frac{t^{2}}{2}}$. Therefore $\log (\phi(t))=-\frac{t^{2}}{2}$, and $\kappa_{1}=0, \kappa_{2}=1$, and $\kappa_{n}=0$ for $n \geq 3$.

The joint cumulants are defined similarly to the joint moments.

Definition 2.13. For a set of random variables $X_{1}, X_{2}, \ldots, X_{k}$, define the function

$$
g\left(t_{1}, t_{2}, \ldots, t_{k}\right)=\log \left[\mathbf{E}\left(e^{i \sum_{j=1}^{k} t_{j} X_{\jmath}}\right)\right], \quad\left(t_{1}, \ldots, t_{k}\right) \in \mathbb{R}^{k}
$$

The joint cumulant $\kappa\left(t_{1}, t_{2}, \ldots, t_{k}\right)$ is the coefficient of $t_{1} t_{2} \ldots t_{k}$ in the Taylor series expansion of $g\left(t_{1}, t_{2}, \ldots, t_{k}\right)$.

Because of the complexity, we shall not discuss the joint cumulants in detail. In what follows, we only need the fourth order cumulant that emerges in Chapter 4 and has the form

$$
\begin{aligned}
\kappa(h, r, s) & =\mathbf{E}\left[\left(X_{k}-\mu\right)\left(X_{k+h}-\mu\right)\left(X_{k+r}-\mu\right)\left(X_{k+s}-\mu\right)\right] \\
& -\left(\gamma_{h} \gamma_{r-s}+\gamma_{r} \gamma_{h-s}+\gamma_{s} \gamma_{h-r}\right) .
\end{aligned}
$$




\subsection{4 $O_{P}(\cdot)$ and $o_{P}(\cdot)$ symbols}

The symbols small "oh" and big "oh", $o(\cdot)$ and $O(\cdot)$, are widely used in Analysis. However, in probability and statistics, when applied to sequences of random variables, they change their meaning.

Definition 2.14. We say that a sequence of random variables $\left\{X_{n}\right\}_{n \geq 1}$ converges to zero in probability, and write $X_{n} \stackrel{P}{\longrightarrow}$, if for every $\epsilon>0, \mathbf{P}\left(\left|X_{n}\right|>\epsilon\right) \rightarrow 0$ as $n \rightarrow \infty$.

Definition 2.15. We say that a sequence of random variables $\left\{X_{n}\right\}_{n \geq 1}$ is bounded in probability, if for every $\epsilon>0$, there exists $\delta=\delta(\epsilon) \in(0, \infty)$, such that $\mathbf{P}\left(\left|X_{n}\right|>\delta\right)<$ $\epsilon$ for all $n=1,2, \ldots$.

Definition 2.16. If $\left\{X_{n}\right\}_{n \geq 1}$ is a sequence of random variables, then:

(a) $X_{n}=o_{P}(1)$ means that $X_{n} \stackrel{P}{\longrightarrow} 0$,

(b) $X_{n}=O_{P}(1)$ means that the sequence $X_{n}$ is bounded in probability.

More generally,

Definition 2.17. If $\left\{R_{n}\right\}_{n \geq 1}$ is the sequence of random variables, then:

(a) $X_{n}=o_{P}\left(R_{n}\right)$ means that $X_{n}=Y_{n} R_{n}$ and $Y_{n} \stackrel{P}{\longrightarrow} 0$,

(b) $X_{n}=O_{P}\left(R_{n}\right)$ means that $X_{n}=Y_{n} R_{n}$ and $Y_{n}=O_{P}(1)$.

In Definition 2.17, the sequence $X_{n}$ converges in probability to zero or is bounded in probability at the "rate" $R_{n}$. There are numerous rules governing $o_{P}(\cdot)$ and $O_{P}(\cdot)$. Let us list some of them without proofs:

(i) $o_{P}(1)+o_{P}(1)=o_{P}(1)$ 
(ii) $o_{P}(1)+O_{P}(1)=O_{P}(1)$;

(iii) $o_{P}\left(R_{n}\right)=R_{n} o_{P}(1)$

(iv) $O_{P}\left(R_{n}\right)=R_{n} O_{P}(1)$

(v) $o_{P}\left(O_{P}(1)\right)=o_{P}(1)$

(vi) $O_{P}(1) o_{P}(1)=o_{P}(1)$

(vii) $\left(1+o_{P}(1)\right)^{-1}=O_{P}(1)$.

The validity of these rules follows from standard continuity results of probability and statistics. For example, relation (i) is a consequence of the continuous mapping theorem, according to which, if $X_{n} \stackrel{P}{\longrightarrow} 0$ and $Y_{n} \stackrel{P}{\longrightarrow} 0$, then $X_{n}+Y_{n} \stackrel{P}{\longrightarrow} 0$. 


\section{Detecting Long Memory through Estimation}

\section{$3.1 \quad \mathrm{R} / \mathrm{S}$ Statistic}

One of the first methods used to distinguish between short and long range dependence in the data was based on the so-called R/S statistics. Using heuristical arguments, this statistic was first derived by Harold E. Hurst (1951), and then refined by Mandelbrot and his collaborators.

We shall take a look at the historical roots, motivation for the $\mathrm{R} / \mathrm{S}$ statistic, and its initial formulation. For further details, see Advances in Geophysics, Vol. 40.

In the 1950's Harold Hurst studied the hydrology of the Nile River. The goal of his research was to determine the storage limits of an ideal reservoir that would never overflow or become empty. He made a few assumptions, namely:

(A) the flow $Q(t)$ into the reservoir is the flow of the river upstream of the dam at time $t$

(B) the flow out of the reservoir, denoted by $\bar{Q}(T)$, is the mean inflow over a period $T$, that is,

$$
\bar{Q}(T)=\frac{1}{T} \int_{0}^{T} Q(t) d t
$$

(C) the initial level of water in the reservoir is $V(0)$.

Under assumptions (A)-(C), the volume of water in the reservoir at any particular time $t \in[0, T]$ is computed by the formula

$$
V(t)=V(0)+\left(\int_{0}^{t} Q(s) d s\right)-t \bar{Q}(T)
$$

For better understanding formula (3.1), note that the flow $Q(t)$ is a physical unit 
which is measured in terms of volume per time. Therefore by multiplying $Q(t)$ by time $t$ or integrating it with respect to $t$, we end up with the volume $V(t)$. At any time $t, V(t)$ equals the initial volume of water in the reservoir plus volume of the inflow over time $t$ minus volume of the outflow over time $t$. Upon substituting $t=T$ into (3.1), we get that the initial volume is equal to the volume at the end of period $T$, i.e., $V(0)=V(T)$. It is important to satisfy the assumption that the reservoir never empties or overflows. For $0<t<T, V(t)$ can vary over time. Its range was defined as the difference between the maximum and minimum volume in the reservoir over the period $T$ and denoted by $R(T)$ :

$$
R(T)=V_{\max }(T)-V_{\min }(T)
$$

Then, the Rescaled Range statistic, in short R/S statistic, was defined as the ratio $\frac{R(T)}{S(T)}$, where $S(T)$ is the standard deviation of the flow $Q(t)$ over the period $T$ given by

$$
S(T)=\left[\frac{1}{T} \int_{0}^{T}\{Q(s)-\bar{Q}(T)\}^{2} d s\right]^{\frac{1}{2}}
$$

The interval $[0, T]$ was split in a sequence into $T / \tau$ subintervals of length $\tau$. One can take $\tau$ to be $\tau=\frac{T}{2}, \frac{T}{4}, \ldots$. For each $\tau$, the values of $R(\tau) / S(\tau)$ were computed for each of the $T / \tau$ subintervals. When calculated, the results were averaged. Hurst (1951) obtained empirically that, as the length $\tau$ of each subinterval goes to zero,

$$
\left(\frac{R(\tau)}{S(\tau)}\right)_{a v} \approx\left(\frac{\tau}{2}\right)^{H}
$$

for some constant $H$ that was later called the Hurst exponent. 
For illustration, suppose we have a sample of size 128. When $\tau=64$, we split the sample into two parts, then compute values of $R(64) / S(64)$ for each part and average them to get $\left(\frac{R(64)}{S(64)}\right)_{a v}$. Continue for $\tau=32,16,8,4,2$ to get the sequence

$$
\left\{\left(\frac{R(64)}{S(64)}\right)_{a v},\left(\frac{R(32)}{S(32)}\right)_{a v},\left(\frac{R(16)}{S(16)}\right)_{a v},\left(\frac{R(8)}{S(8)}\right)_{a v},\left(\frac{R(4)}{S(4)}\right)_{a v},\left(\frac{R(2)}{S(2)}\right)_{a v}\right\} .
$$

Using (3.2), the constant $H$ can be estimated by plotting $\log \left(\frac{R(\tau)}{S(\tau)}\right)_{a v}$ against $\log (\tau / 2)$ and finding the best fitting line.

We now give the modern formulation of the $\mathrm{R} / \mathrm{S}$ statistic. The statistic is defined as the range of partial sums of deviations of a time series from its mean, rescaled by its standard deviation, that is,

$$
R / S=\frac{1}{\hat{\sigma}_{n}}\left[\max _{1 \leq k \leq n} \sum_{j=1}^{k}\left(X_{j}-\bar{X}_{n}\right)-\min _{1 \leq k \leq n} \sum_{j=1}^{k}\left(X_{j}-\bar{X}_{n}\right)\right]
$$

where $\hat{\sigma}_{n}$ is a regular sample standard deviation given by

$$
\hat{\sigma}_{n}=\left[\frac{1}{n} \sum_{j=1}^{n}\left(X_{j}-\bar{X}_{n}\right)^{2}\right]^{1 / 2} .
$$

Then, the long memory parameter $d=H-1 / 2$ can be estimated by

$$
\hat{d}=\frac{\log (R / S)}{\log (n)}-\frac{1}{2}
$$

Indeed, taking the logarithm on both sides of (3.2), we get the estimator for $H$ of the form

$$
\hat{H}=\frac{\log (R(n) / S(n))}{\log (n)-\log (1 / 2)} \approx \frac{\log (R / S)}{\log (n)} .
$$


From this equality (3.3) follows immediately.

Mandelbrot and Wallis (1969) showed the robustness of R/S statistic to nonGaussian distributions, and Mandelbrot (1975) reported the robustness to distributions with infinite variance. Nevertheless, the R/S statistic has a serious drawback. It is sensitive to short range dependence. The test based on this statistic often identifies long memory in cases, where in fact only short memory is present. Although initially a long memory test based on the $\mathrm{R} / \mathrm{S}$ statistic was very popular, nowadays its use is very limited. In 1991, Lo suggested a modified version of the R/S statistic, which addresses the issue of short range sensitivity. Lo's modification will be considered in Section 4.1.

$\mathrm{R}$ code for computing $\mathrm{R} / \mathrm{S}$ statistic is given in the Appendix.

\subsection{Variance Plots}

From Section 2.2.4, we know that the autocovariance function of a long-memory time series has the form

$$
\gamma(k) \sim c_{\gamma} k^{2 H-2}, \quad \text { for large } k
$$

Also, for the sample mean $\bar{X}=n^{-1}\left(X_{1}+\ldots+X_{n}\right)$, one has

$$
\begin{aligned}
\operatorname{Var}(\bar{X}) & =\frac{1}{n^{2}} \sum_{t=1}^{n} \sum_{s=1}^{n} \operatorname{Cov}\left(X_{t}, X_{s}\right)=\frac{1}{n^{2}} \sum_{t=1}^{n} \sum_{s=1}^{n} \gamma(t-s) \\
& =\frac{1}{n^{2}} \sum_{k=-(n-1)}^{n-1}(n-|k|) \gamma(k)=\frac{1}{n} \sum_{k=-(n-1)}^{n-1}\left(1-\frac{|k|}{n}\right) \gamma(k) \\
& =\frac{2}{n}\left[\sum_{k=1}^{n-1}\left(1-\frac{k}{n}\right) \gamma(k)+\frac{\gamma(0)}{2}\right]
\end{aligned}
$$


Therefore, for large $n$

$$
\begin{aligned}
\operatorname{Var}(\bar{X}) & \sim 2 c_{\gamma} n^{2 H-2} \sum_{k=1}^{n-1}\left[\left(1-\frac{k}{n}\right)\left(\frac{k}{n}\right)^{2 H-2} \frac{1}{n}\right]=2 c_{\gamma} n^{2 H-2} \int_{0}^{1}(1-t) t^{2 H-2} d t \\
& =2 c_{\gamma} n^{2 H-2}\left(\int_{0}^{1} t^{2 H-2} d t-\int_{0}^{1} t^{2 H-1} d t\right)=2 c_{\gamma} n^{2 H-2}\left(\frac{1}{2 H-1}-\frac{1}{2 H}\right) \\
& =2 c_{\gamma} n^{2 H-2} \frac{2 H-(2 H-1)}{2 H(2 H-1)}=\frac{c_{\gamma} n^{2 H-2}}{H(2 H-1)}
\end{aligned}
$$

It is well known that the variance of the sample mean $\bar{X}$ for short memory time series decays at the rate $n^{-1}$. It follows from (3.4) that for a long memory time series, the variance of $\bar{X}$ converges to zero at a rate slower than $n^{-1}$. Based on this phenomenon, an original method, called the varance plot method for the detection of long-range dependence was constructed.

Here is its basic description. Assume that a sample of size $n$ is available. Divide it into $m_{k}$ blocks of size $k$, so that $n=m_{k} \times k$. Also, let $k$ be an integer number satisfying $2 \leq k \leq\left[\frac{n}{2}\right]$. Then for $j=1,2, \ldots, m_{k}$, calculate the averages $\bar{X}_{1}(k), \bar{X}_{2}(k), \ldots, \bar{X}_{m_{k}}(k)$ of all blocks separately as

$$
\bar{X}_{\jmath}(k)=k^{-1} \sum_{t=(\jmath-1) \times k+1}^{\jmath \times k} X_{t}
$$

and also the overall mean

$$
\bar{X}(k)=m_{k}^{-1} \sum_{j=1}^{m_{k}} \bar{X}_{\jmath}(k)
$$

After that, calculate the sample variances of the sequence $\bar{X}_{1}(k), \bar{X}_{2}(k), \ldots, \bar{X}_{m_{k}}(k)$ 
for each $k \in[2,[n / 2]]$ by the formula

$$
S^{2}(k)=\left(m_{k}-1\right)^{-1} \sum_{\jmath=1}^{m_{k}}\left(\bar{X}_{\jmath}(k)-\bar{X}(k)\right)^{2}
$$

and plot $S^{2}(k)$ against $\log (k)$.

For large $k$, points on the plot will become concentrated around a straight line with a negative slope. For short memory or independent time series, the slope will be at least as steep as -1 . Usually, for short range dependent data, slope will be steeper. For long range dependent data, on the contrary, the slope will be bigger than -1 . The method is visual and compelling but rough. It can only identify long memory when the evidence is very strong.

Using the regression technique and the form of variance of the sample mean for long memory time series (see (3.4)), it is possible to estimate the Hurst exponent. To do this, observe that for the $j$ th block, as $k \rightarrow \infty$

$$
\operatorname{Var}\left(\bar{X}_{\jmath}\right) \sim c k^{2 H-2} \Rightarrow \log \left(\operatorname{Var}\left(\bar{X}_{\jmath}\right)\right) \sim c+(2 H-2) \log k
$$

Denote $x_{\jmath}=\log k$ and $y_{\jmath}=\log \left(\operatorname{Var}\left(\bar{X}_{\jmath}\right)\right)$. Then the relation in (3.5) can be approximated by the relation

$$
y_{\jmath}=c+(2 H-2) x_{\jmath}
$$

Using the ordinary least square estimator, the slope $2 H-2$ can be estimated by

$$
2 \hat{H}-2=-\frac{\sum_{\jmath=1}^{m_{k}}\left(x_{\jmath}-\bar{x}\right)\left(y_{\jmath}-\bar{y}\right)}{\sum_{\jmath=1}^{m_{k}}\left(x_{\jmath}-\bar{x}\right)^{2}}
$$


and hence

$$
\hat{H}=1-\frac{\sum_{j=1}^{m_{k}}\left(\log k-\frac{\sum_{j=1}^{m_{k}} x_{j}}{m_{k}}\right)\left(\log \left(\operatorname{Var}\left(\bar{y}_{j}\right)\right)-\frac{\sum_{j=1}^{m_{k}} \log \left(\operatorname{Var}\left(\bar{y}_{j}\right)\right)}{m_{k}}\right)}{2 \sum_{j=1}^{m_{k}}\left(\log k-\frac{\sum_{j=1}^{m_{k}} \log k}{m_{k}}\right)^{2}} .
$$

\subsection{Geweke and Porter-Hudak Estimator}

In the seminal work of Geweke and Porter-Hudak (1983), another widely used estimator of the self-similarity parameter was introduced. To denote the Geweke and Porter-Hudak estimator in papers, a short abbreviation "GPH" is often used. For consistency, we keep this abbreviation in the thesis.

Geweke and Porter-Hudak considered a general integrated series model

$$
(1-B)^{d} X_{t}=u_{t}, \quad t \in \mathbb{Z}
$$

where $\left\{u_{t}\right\}$ is a stationary linear process with spectral density function $f_{u}(\lambda)$. It follows from Section 2.2.6 that the spectral density of this model behaves itself around $\lambda=0$ as follows:

$$
f_{X}(\lambda) \approx|\lambda|^{-2 d} f_{u}(\lambda)
$$

Taking the logarithm of both sides of (3.6), we get

$$
\ln f_{X}(\lambda)=\ln f_{u}(0)-d \ln \lambda^{2}+\ln \frac{f_{u}(\lambda)}{f_{u}(0)}
$$


Since $\ln \frac{f_{u}(\lambda)}{f_{u}(0)} \rightarrow 0$ as $\lambda \rightarrow 0$, we can write

$$
\ln f_{X}(\lambda) \approx \ln f_{u}(0)-d \ln \lambda^{2}
$$

Let $\lambda_{\jmath}$ be the Fourier frequencies $\lambda_{\jmath}=\frac{2 \pi j}{n}$ for $j \in(0, \pi)$, and let $I_{n}\left(\lambda_{\jmath}\right)$ be the periodogram of the integrated process $\left\{X_{t}\right\}$ at frequency $\lambda_{\jmath}$. Then adding $\ln I_{n}\left(\lambda_{\jmath}\right)$ to both sides of (3.8), we get

$$
\ln I_{n}\left(\lambda_{\jmath}\right) \approx \ln f_{u}(0)-d \ln \lambda_{\jmath}^{2}+\ln \frac{I_{n}\left(\lambda_{\jmath}\right)}{f_{X}\left(\lambda_{\jmath}\right)}
$$

We can now treat relation (3.9) approximately as a regression

$$
Y_{\jmath}=a+b x_{\jmath}+\epsilon_{\jmath}
$$

where

$$
Y_{\jmath}=\ln I_{n}\left(\lambda_{\jmath}\right), \quad a=\ln f_{u}(0), \quad b=-d, \quad x_{\jmath}=\ln \lambda_{\jmath}^{2}, \quad \epsilon_{\jmath}=\ln \frac{I_{n}\left(\lambda_{\jmath}\right)}{f_{X}\left(\lambda_{\jmath}\right)}
$$

When estimating $d$ by using the ordinary least square (OLS) technique, we get

$$
\hat{d}=-\frac{\sum_{\jmath=1}^{m}\left(x_{\jmath}-\bar{x}\right)\left(Y_{\jmath}-\bar{Y}\right)}{\sum_{\jmath=1}^{m}\left(x_{\jmath}-\bar{x}\right)^{2}},
$$

where $\bar{x}$ and $\bar{Y}$ are the sample means of $x$ and $Y$ defined above. The parameter $m$ is a window. It is chosen to have $m / n \rightarrow 0$ as $n \rightarrow \infty$ and $m \rightarrow \infty$, and is usually taken to be $m=\sqrt{n}$.

Notice that Geweke and Porter-Hudak (1983) used slightly different form of spec- 
tral density for $\operatorname{FARIMA}(p, d, q)$, namely

$$
f(\lambda)=\left\{4 \sin ^{2} \frac{\lambda}{2}\right\}^{-d} f_{u}(\lambda)
$$

The spectral density in (3.6) that we used in our calculations is an approximation of the spectral function in (3.11) for $\lambda$ around zero. Although later on, in our computation of the GPH estimator, we assume $\lambda_{\jmath}$ to be the Fourier frequencies, the approximation of (3.11) is still valid. This is so because for $j=1, \ldots, m$, we normally choose $m=\sqrt{n}$ and have

$$
\max _{\jmath} \lambda_{\jmath}=\frac{2 \pi \sqrt{n}}{n}=\frac{2 \pi}{\sqrt{n}} \rightarrow 0, \quad \text { as } \quad n \rightarrow \infty .
$$

In their seminal paper, Geweke and Porter-Hudak (1983) studied consistency of the estimator $\hat{d}$ of $d$ for $-1 / 2 \leq d \leq 0$, and found that $\hat{d}$ is asymptotically normal. More precisely, as $n \rightarrow \infty$

$$
\hat{d} \stackrel{D}{\longrightarrow} N\left(d, \frac{\pi^{2}}{6 \sum_{\jmath=1}^{m}\left(x_{\jmath}-\bar{x}\right)^{2}}\right) .
$$

They also formulated a conjecture that the convergence in (3.12) remains valid for $d>0$, and justified this conjecture by simulation.

$\mathrm{R}$ code for computing the value of Geweke and Porter-Hudak estimator is given in the Appendix.

\subsection{Whittle Estimator}

A Whittle estimator is based on the likelihood function of a Gaussian process. It does not mean, however, that this estimator cannot be used for non-Gaussian processes. 
Jan Beran (1994) pointed out in his book that, at least for some of the methods, analogs of the same central limit are known to hold for more general processes. However, if applied to non-Gaussian processes, some caution is needed.

A Whittle estimator was developed by Künsch (1987) and Robinson (1995). Suppose that $X_{1}, X_{2}, \ldots, X_{n}$ is a stationary process with mean $\mu$ and variance $\sigma^{2}$. We also assume that $0<d<1 / 2$ the process has a long memory property in the sense of Definition 2.9. Let $\mathbf{X}=\left(X_{1}, \ldots, X_{n}\right)^{\top}$ be the sample vector of this process. Also, let $\Theta=\left(\mu, \sigma^{2}, d, \theta_{4}, \ldots, \theta_{m}\right)$ be a vector of unknown parameters. So, we may assume that the spectral density comes from a parametric family $f(\lambda)=f(\lambda ; \theta)$, where $\theta$ is a vector in $\mathbb{R}^{m}$. We denote the covariance matrix of $\mathbf{X}$ by $\Sigma(\Theta)$ and its determinant by $|\Sigma(\Theta)|$. The Gaussian log likelihood function has the form

$$
\Lambda(\Theta)=-\frac{1}{2 n} \log |\Sigma(\Theta)|-\frac{1}{2 n} \mathbf{X}^{\top} \Sigma^{-1}(\Theta) \mathbf{X}
$$

In order to estimate the integration parameter $d$, we need to maximize $\Lambda(\Theta)$, or equivalently, to minimize the function

$$
Q(\Theta)=\frac{1}{2 n} \log |\Sigma(\Theta)|+\frac{1}{2 n} \mathbf{X}^{\top} \Sigma^{-1}(\Theta) \mathbf{X}
$$

It was shown by Grenader and Szegö (1958) that

$$
\lim _{n \rightarrow \infty} \frac{1}{n} \log |\Sigma(\Theta)|=\frac{1}{2 \pi} \int_{-\pi}^{\pi} \log f(\lambda ; \Theta) d \lambda
$$

The inverse of the covariance matrix $\Sigma^{-1}(f)$ of some positive function $f$ can be approximated by the covariance matrix of the inverse of that function, namely, $\Sigma\left(f^{-1}\right)$. (Recall that the covariance matrix $\Sigma(f)$ is defined as the matrix $(\gamma(k-l))_{k, l=1}^{n}$, where 
$\gamma(k-l)=\int_{-\pi}^{\pi} e^{\imath(k-l) \lambda} f(\lambda) d \lambda$. See, for example, Dahlhaus (1989) for details.) Künsch (1987) showed that the covariance matrix $\Sigma^{-1}(\Theta)$ can be approximated by the $n \times n$ matrix $\mathbf{A}$ with the $(j, k)$ th entry $A_{j k}, j, k=1, \ldots n$, equal to

$$
A_{\jmath k}=\frac{1}{4 \pi^{2}} \int_{-\pi}^{\pi} \frac{1}{f(\lambda ; \Theta)} e^{\imath(\jmath-k) \lambda} d \lambda
$$

Using these two approximations, Künsch (1987) concluded that the negative of log likelihood given by (3.13) satisfies

$$
Q(\Theta) \approx \frac{1}{m} \sum_{\jmath=0}^{m}\left[\log \left(f\left(\lambda_{\jmath}\right)\right)+\frac{I\left(\lambda_{\jmath}\right)}{f\left(\lambda_{\jmath}\right)}\right]
$$

where $I\left(\lambda_{\jmath}\right)$ is the periodogram

$$
I_{\jmath}=I\left(\lambda_{\jmath}\right)=2 \pi n^{-1}\left|\sum_{\jmath=1}^{n} e^{\imath \jmath \lambda_{\jmath}} X_{\jmath}\right|^{2}
$$

and $\lambda_{\jmath}$ are the Fourier frequencies $\lambda_{\jmath}=\frac{2 \pi j}{n}$ with $j=1, \ldots, \frac{[n-1]}{2}$.

In the paper of Künsch (1987) the derivation of this statement is omitted. We shall present here the derivation of (3.16) in detail.

First, consider the approximation given by (3.15). We have

$$
\begin{gathered}
\frac{1}{n} \mathbf{X}^{\top} \mathbf{A X}=\frac{1}{2 \pi} \frac{1}{2 \pi n} \mathbf{X}^{\top}\left(\int_{-\pi}^{\pi} e^{-\imath \lambda(\jmath-k)} \frac{1}{f(\lambda)} d \lambda\right)_{\jmath k} \mathbf{X}=\frac{1}{2 \pi} \int_{-\pi}^{\pi}\left(\frac{1}{2 \pi n} \mathbf{X}^{\top} e^{-\imath \lambda(\jmath-k)} \mathbf{X}\right) \frac{1}{f(\lambda)} d \lambda \\
=\frac{1}{2 \pi} \int_{-\pi}^{\pi}\left(\frac{1}{2 \pi n} \mathbf{X}^{\top} e^{-\imath \lambda(\jmath-k)} \mathbf{X}\right) \frac{1}{f(\lambda)} d \lambda=\frac{1}{2 \pi} \int_{-\pi}^{\pi} \frac{1}{2 \pi n} \sum_{k=1}^{n} \sum_{\jmath=1}^{n}\left(X_{k} e^{\imath \lambda_{k}}\right)\left(X_{\jmath} e^{-\imath \lambda_{\jmath}}\right) \frac{1}{f(\lambda)} d \lambda \\
=\frac{1}{2 \pi} \int_{-\pi}^{\pi} \frac{1}{2 \pi n}\left|\sum_{\jmath=1}^{n} X_{\jmath} e^{\imath \lambda_{\jmath}}\right|^{2} \frac{1}{f(\lambda)} d \lambda=\frac{1}{2 \pi} \int_{-\pi}^{\pi} \frac{I_{n}(\lambda)}{f(\lambda)} d \lambda .
\end{gathered}
$$


Therefore as $n \rightarrow \infty$,

$$
Q(\Theta) \approx \frac{1}{2 \pi} \int_{-\pi}^{\pi} \log f(\lambda) d \lambda+\frac{1}{2 \pi} \int_{-\pi}^{\pi} \frac{I_{n}(\lambda)}{f(\lambda)} d \lambda
$$

We can neglect by the term $1 / 2 \pi$ because it does not effect the minimization of $Q(\Theta)$. We shall keep the same notation for $Q(\Theta)$ with the factor $2 \pi$ omitted. Thus

$$
Q(\Theta) \approx \int_{-\pi}^{\pi} \log f(\lambda) d \lambda+\int_{-\pi}^{\pi} \frac{I_{n}(\lambda)}{f(\lambda)} d \lambda
$$

The next step is to use the discretization of integrals. We have

$$
\begin{aligned}
Q(\Theta) & \approx \lim _{n \rightarrow \infty} \sum_{\jmath=1}^{n} \log f\left(\lambda_{\jmath}\right)\left(\lambda_{\jmath}-\lambda_{\jmath-1}\right)+\lim _{n \rightarrow \infty} \sum_{\jmath=1}^{n} \frac{I_{n}\left(\lambda_{\jmath}\right)}{f\left(\lambda_{\jmath}\right)}\left(\lambda_{\jmath}-\lambda_{\jmath-1}\right) \\
& =\lim _{n \rightarrow \infty} \frac{1}{n} \sum_{\jmath=1}^{n}\left(\log f\left(\lambda_{\jmath}\right)+\frac{I_{n}\left(\lambda_{\jmath}\right)}{f\left(\lambda_{\jmath}\right)}\right) .
\end{aligned}
$$

Since $n \rightarrow \infty$, we can take any $m$ such that $n^{\varepsilon} \leq m \leq n^{1-\varepsilon}$ in place of $n$ in (3.17), and still get a good approximation of the integration. From this, relation (3.16) follows.

We now move on to the derivation of a Whittle estimator of $d$. Our computations will be based on the paper of Robinson (1995). As most expansions in this paper are not detailed, we shall try to clarify difficult moments and fill in the blanks in the proof.

Recall that the spectral density of $\operatorname{FARIMA}(p, d, q)$ long memory time series is $f(\lambda)=G|\lambda|^{-2 d},-\pi \leq \lambda \leq \pi$, where $G$ is defined in (2.14). Following Robinson (1995), we can rewrite $Q(\Theta)$ in the form

$$
Q(G, d)=\frac{1}{m} \sum_{\jmath=1}^{m}\left\{\log G \lambda^{-2 d}+\frac{\lambda^{2 d}}{G} I_{\jmath}\right\}=\log G-\frac{2 d}{m} \sum_{\jmath=1}^{m} \log \lambda_{\jmath}+\frac{\frac{1}{m} \sum_{\jmath=1}^{m} \lambda_{\jmath}^{2 d} I_{\jmath}}{G} .
$$


In order to find the argument $G$ that minimizes $Q(G, d)$, we take the derivative of $Q(G, d)$ with respect to $G$ and set it equal to zero:

$$
\frac{\partial Q(G, d)}{\partial G}=\frac{1}{G}-0-\frac{1}{m G^{2}} \sum_{\jmath=1}^{m} \lambda_{\jmath}^{2 d} I_{\jmath}=0
$$

Solution to this equation has the form

$$
\hat{G}=\frac{\sum_{j=1}^{m} \lambda_{\jmath}^{2 d} I_{\jmath}}{m} .
$$

Now, in the expression for $Q(G, d)$, we substitute $\hat{G}$ in place of $G$ and get

$$
Q(\hat{G}, d)=\log \hat{G}-\frac{2 d}{m} \sum_{\jmath=1} m \log \lambda_{\jmath}+\frac{\frac{1}{m} \sum_{\jmath=1}^{m} \lambda_{\jmath}^{2 d} I_{\jmath}}{\hat{G}} .
$$

If we care only about finding arguments that minimize $Q(G, d)$, adding a constant in $Q(G, d)$ becomes not important. Therefore, since

$$
\frac{\frac{1}{m} \sum_{\jmath=1}^{m} \lambda_{\jmath}^{2 d} I_{\jmath}}{\hat{G}}=1
$$

we can neglect it and keep using $Q(G, d)$. Finally, we get

$$
\begin{aligned}
Q(\hat{G}, d) & =\log \left(\frac{1}{m} \sum_{\jmath=1}^{m} \lambda_{\jmath}^{2 d} I_{\jmath}\right)-\frac{2 d}{m} \sum_{\jmath=1}^{m} \log \lambda_{\jmath}=\log \left(\frac{1}{m} \sum_{\jmath=1}^{m}\left(\frac{2 \pi j}{n}\right)^{2 d} I_{\jmath}\right) \\
& -\frac{1}{m} \sum_{\jmath=1}^{m} \log \left(\frac{2 \pi j}{n}\right)^{2 d}=\log \frac{2 \pi}{n}+\log \left(\frac{1}{m} \sum_{\jmath=1}^{m} j^{2 d} I_{\jmath}\right)-\frac{1}{m} \sum_{\jmath=1}^{m} \log \frac{2 \pi}{n} \\
& -\frac{1}{m} \sum_{\jmath=1}^{m} \log j^{2 d}=\log \left(\frac{1}{m} \sum_{\jmath=1}^{m} j^{2 d} I_{\jmath}\right)-\frac{1}{m} \sum_{\jmath=1}^{m} \log j^{2 d},
\end{aligned}
$$


where $\left\{\lambda_{\jmath}\right\}_{\jmath=1, \ldots[n-1] / 2}$ is a sequence of the Fourier frequencies, $I_{J}$ is the periodogram of long memory process $\left\{X_{t}\right\}$ at $\lambda_{\jmath}$, and $m$ is a window parameter satisfying $n^{\varepsilon} \leq$ $m \leq n^{1-\varepsilon}$. A natural estimator of the long memory parameter $d$ is

$$
\hat{d}=\arg \min _{d \in(-0.5,0.5)} Q(\hat{G}, d)
$$

This estimator, called a Whittle estimator, was suggested by Robinson (1995). Robinson showed its consistency for $d \in(-0.5,0.5)$, and also established its asymptotic normality:

$$
\sqrt{m}(\hat{d}-d) \stackrel{D}{\longrightarrow} N\left(0, \frac{1}{4}\right)
$$

My experience shows that the estimator $\hat{d}$ in (3.18) is the most precise and comfortable for use, out of the three estimators discussed so far. In addition, not only it can be used to estimate a long memory parameter $d$ when $d>0$, but also to identify a negative memory for $d<0$. R code for computing the value of Whittle estimator is given in the Appendix. 


\section{Detecting Long Memory through Testing}

\subsection{Modified R/S Statistic}

\subsubsection{Definition of Modified R/S Statistic}

It was proved by Lo (1991) that the R/S test statistic is not robust against short range dependence. More specifically, in case of short memory, R/S will converge to a rescaled range: $\sigma / \sqrt{\gamma(0)}$ times the range of Brownian Bridge, where $\sigma^{2}$ is the sum of all covariances. That is, as a test statistic, $\mathrm{R} / \mathrm{S}$ is very sensitive to small deviations from independence. Such deviations will be seen as a long memory pattern. To accommodate for short range dependence, Lo has proposed a modified R/S statistic which is defined as follows.

Let $\hat{\gamma}_{3}$ denote the sample covariance

$$
\hat{\gamma}_{0}=\frac{1}{n} \sum_{\jmath=1}^{n}\left(X_{\jmath}-\bar{X}\right)^{2}, \quad \hat{\gamma}_{\jmath}=\frac{1}{n} \sum_{\imath=1}^{n-\jmath}\left(X_{\imath}-\bar{X}\right)\left(X_{\imath+\jmath}-\bar{X}\right), \quad 1 \leq j \leq n .
$$

Put $\bar{X}=\frac{1}{n} \sum_{j=1}^{n} X_{\jmath}$ and $\hat{\sigma}_{n, q}^{2}=\hat{\gamma}_{0}+2 \sum_{\jmath=1}^{q} \omega_{\jmath}(q) \hat{\gamma}_{\jmath}$, where $\omega_{\jmath}(q)=1-\frac{j}{q+1}$ for $q \sim$ $o_{P}\left(n^{1 / 4}\right)$, with $q$ being a parameter that may be data dependent. Note: there should be no confusion between this window $q$, which is a subsequence of $n$, and the other $q$ in $\operatorname{MA}(q)$ (order of moving average polynomial) that appeared in the beginning of the thesis.

Lo's statistic is given by

$$
R / S_{\text {mod }}=\frac{1}{\hat{\sigma}_{n, q}}\left[\max _{1 \leq k \leq n} \sum_{\jmath=1}^{k}\left(X_{\jmath}-\bar{X}\right)-\min _{1 \leq k \leq n} \sum_{\jmath=1}^{k}\left(X_{\jmath}-\bar{X}\right)\right] .
$$

As seen from (4.1), the numerator of $R / S_{\text {mod }}$ is the same as that of $R / S$, which 
is discussed in Section 3.1. The denominator of $R / S_{m o d}$ is the square root of a consistent estimator of the partial sum's variance. If the sequence $\left\{X_{t}\right\}$ constitutes a short memory process, the variance of a partial sum is not simply the sum of the variances of the individual terms, but also involves the autocovariances up to lag $q$.

The choice of an optimal parameter $q$ was studied by Andrews (1991). Based on Andrews' work, Lo (1991) suggested the optimal data dependent choice of $q$, which was valid only for an autoregressive AR(1) processes, namely,

$$
q=\left[k_{n}\right], \quad k_{n}=\left(\frac{3 n^{2}}{2}\right)\left(\frac{2 \hat{\rho}}{1-\hat{\rho}^{2}}\right)^{\frac{3}{2}}
$$

where $\left[k_{n}\right]$ is the greatest integer less than or equal to $k_{n}$.

\subsubsection{Testing Short Memory versus Long Memory Using Modified R/S Statistic}

We now look at the limiting behaviour of $R / S_{\text {mod }}$ under the hypotheses of short and long memory.

\section{a) Asymptotic behaviour of $R / S_{\text {mod }}$ under short memory.}

In his paper, Lo (1991) proved that under the short memory null hypothesis, assuming that the cumulants satisfy

$$
\sup _{h} \sum_{r, s=-\infty}^{\infty}|k(h, r, s)|<\infty
$$

the normalized modified R/S statistic is weakly convergent:

$$
\frac{R / S_{\bmod }}{\sqrt{n}} \stackrel{D}{\longrightarrow} Q, \quad n \rightarrow \infty
$$


where

$$
Q=\sup _{0 \leq t \leq 1} W^{0}(t)-\inf _{0 \leq t \leq 1} W^{0}(t)
$$

is the range of a Brownian bridge. Consequently, $R / S_{\text {mod }}$ can be used to test the short memory null hypothesis against long memory alternatives. An exact form of the distribution function of $Q$ is known to be

$$
\mathbf{F}_{Q}(x)=\mathbf{P}(Q \leq x)=1+2 \sum_{\jmath=1}^{\infty}\left(1-4 j^{2} x^{2}\right) e^{-2 \jmath^{2} x^{2}}, \quad x \in \mathbb{R}
$$

and

$$
\mathbf{E}(Q)=\frac{\pi}{2}, \quad \operatorname{Var}(Q)=\frac{\pi(\pi-3)}{6}
$$

For details, see Feller (1951). The quantiles of this distribution, which were reported by Lo (1991), are given below.

Table 2: Quantile values of the limiting c.d.f. of the modified R/S statistic.

\begin{tabular}{ccccccccc}
\hline \hline $\mathbf{P}(Q \leq x)$ & 0.005 & 0.025 & 0.050 & 0.100 & 0.200 & 0.300 & 0.400 & 0.500 \\
\hline$x$ & 0.721 & 0.809 & 0.861 & 0.927 & 1.018 & 1.090 & 1.157 & 1.223 \\
\hline $\mathbf{P}(Q \leq x)$ & 0.543 & 0.600 & 0.700 & 0.800 & 0.900 & 0.950 & 0.975 & 0.995 \\
\hline$x$ & $\sqrt{\frac{\pi}{2}}$ & 1.294 & 1.374 & 1.473 & 1.620 & 1.747 & 1.862 & 2.098 \\
\hline
\end{tabular}

b) Asymptotic behaviour of $R / S_{\text {mod }}$ under the alternative of long memory. Lo (1991) also established the weak convergence of $R / S_{\text {mod }}$ under the long memory hypothesis

$$
H_{a}: \quad X_{t}=\mu+\varepsilon_{t}
$$


where $\left\{\varepsilon_{t}\right\}$ is a zero mean Gaussian process whose autocovariance function is

$$
\gamma_{k}=k^{2 d-1} L(k), \quad \text { as } \quad k \rightarrow \infty,
$$

with $L(k)$ being a slowly varying function at infinity. By putting an extra condition on the cumulants of the underlying process, namely,

$$
\sup _{h} \sum_{r, s=-n}^{n}|k(h, r, s)|=O\left(n^{2 d}\right)
$$

Lo proved that the normalized statistic $R / S_{\text {mod }}$ is weakly convergent:

$$
\left(\frac{q}{n}\right)^{d} \frac{R / S_{m o d}}{\sqrt{n}} \stackrel{D}{\longrightarrow} \tilde{Q}, \quad n \rightarrow \infty
$$

where $\tilde{Q}=\sup _{0 \leq t \leq 1} W_{1 / 2+d}^{0}(t)-\inf _{0 \leq t \leq 1} W_{1 / 2+d}^{0}(t)$ is the range of a fractional Brownian bridge. The above condition on the cumulants ensures that the underlying process does not deviate from the normality too far. For definition of cumulants, see Section 2.3.3.

We now examine more closely the limiting behavior of $R / S_{\text {mod }}$ under the short memory null hypothesis. For this, let $k$ be an integer such that $k \leq n t<k+1$, where $[n t]$ is an integer less than or equal to $n t$ for $t \in[0,1]$. Then

$$
\max _{1 \leq k \leq n} \sum_{j=1}^{k}\left(X_{\jmath}-\bar{X}\right)-\min _{1 \leq k \leq n} \sum_{j=1}^{k}\left(X_{\jmath}-\bar{X}\right)=\sup _{0 \leq t \leq 1} \sum_{\imath=1}^{[n t]}\left(X_{\imath}-\bar{X}\right)-\inf _{0 \leq t \leq 1} \sum_{\imath=1}^{[n t]}\left(X_{\imath}-\bar{X}\right)
$$

Therefore we can write

$$
R / S_{\text {mod }}=\left(\sup _{0 \leq t \leq 1} \sum_{\imath=1}^{[n t]} \frac{\left(X_{\imath}-\bar{X}\right)}{\sigma \sqrt{n}}-\inf _{0 \leq t \leq 1} \sum_{\imath=1}^{[n t]} \frac{\left(X_{\imath}-\bar{X}\right)}{\sigma \sqrt{n}}\right) \frac{\sigma \sqrt{n}}{\hat{\sigma}_{n, q}}
$$


From Section 2.3.2 we know that

$$
\sup _{0 \leq t \leq 1} \sum_{\imath=1}^{[n t]} \frac{\left(X_{\imath}-\bar{X}\right)}{\sigma \sqrt{n}}-\inf _{0 \leq t \leq 1} \sum_{\imath=1}^{[n t]} \frac{\left(X_{\imath}-\bar{X}\right)}{\sigma \sqrt{n}} \stackrel{D}{\longrightarrow} \sup _{0 \leq t \leq 1} W^{0}(t)-\inf _{0 \leq t \leq 1} W^{0}(t) .
$$

Also, by Theorem 7.2.2 of Brockwell (1991) for stationary time series,

$$
\sqrt{n}\left(\hat{\rho}_{\jmath}-\rho_{\jmath}\right) \stackrel{D}{\longrightarrow} N\left(0, \sigma_{\jmath}\right), \quad n \rightarrow \infty
$$

where the $\hat{\rho}_{g} \mathrm{~s}$ and $\rho_{j} \mathrm{~s}$ are the sample and population autocorrelations, respectively, and the $\sigma_{\jmath}$ s are the Bartlett coefficients. Normalizing the expression in (4.2) by $\sqrt{n}$, we get

$$
\frac{1}{\sqrt{n}}\left[\sqrt{n}\left(\hat{\gamma}_{j}-\gamma_{\jmath}\right)\right] \stackrel{P}{\longrightarrow} 0
$$

where the $\hat{\gamma}_{g}$ s and $\gamma_{g}$ s are the sample and population autocovariances, respectively. This implies

$$
\hat{\gamma}_{\jmath} \stackrel{P}{\longrightarrow} \gamma_{\jmath}, \quad n \rightarrow \infty
$$

Therefore, taking into account that $\omega_{\jmath}(q)=1-\frac{\jmath}{q+1} \rightarrow 1$ as $q \rightarrow \infty$,

$$
\hat{\sigma}_{n, q}^{2}=\hat{\gamma}_{0}+2 \sum_{\jmath=1}^{q} \omega_{\jmath}(q) \hat{\gamma}_{\jmath} \stackrel{P}{\longrightarrow} \sum_{\jmath=-\infty}^{\infty} \gamma_{\jmath}=\sigma^{2}
$$

Hence

$$
\frac{\sigma}{\hat{\sigma}_{n, q}} \stackrel{P}{\longrightarrow} 1
$$


and by Slutsky's lemma,

$$
\frac{R / S_{m o d}}{\sqrt{n}} \stackrel{D}{\longrightarrow} \sup _{0 \leq t \leq 1} W^{0}(t)-\inf _{0 \leq t \leq 1} W^{0}(t) .
$$

$\mathrm{R}$ code for computing the value of $R / S_{\text {mod }}$ can be found in the Appendix.

\subsection{KPSS Statistic}

A KPSS statistic was introduced by Kwiatkowski et al. in 1992 for testing a trend stationarity against the unit root alternative. Later on, several papers have been written to extend the use of KPSS statistic to other cases, including:

(a) testing the short memory null hypothesis versus the long memory alternative, Lee and Smidt (1996);

(b) testing the stationarity null hypothesis versus nonstationary long memory alternatives, Lee and Amsler (1997).

The KPSS statistic is given by the formula

$$
K_{n}(q)=\frac{1}{n^{2} \hat{\sigma}_{n, q}^{2}} \sum_{k=1}^{n}\left(\sum_{j=1}^{k}\left(X_{j}-\bar{X}\right)\right)^{2}
$$

where $\hat{\sigma}_{n, q}^{2}$ is the same as in the definition of $R / S_{\text {mod }}$. Consider the asymptotic behaviour of $K_{n}(q)$ under the short memory and long memory hypotheses.

Under the null hypothesis of short memory stationarity in the data, assuming that the cumulants satisfy

$$
\sup _{h} \sum_{r, s=-\infty}^{\infty}|k(h, r, s)|<\infty
$$


the KPSS statistic is weakly convergent:

$$
K_{n}(q) \stackrel{D}{\longrightarrow} U_{K P S S}, \quad n \rightarrow \infty
$$

where $U_{K P S S}=\int_{0}^{1}\left(W^{0}(t)\right)^{2} d t$ with $W^{0}(t)$ being a Brownian bridge.

In 1959, Kiefer determined the exact form of the limiting cumulative distribution function (c.d.f.) of $U_{K P S S}$ and gave quantiles of this limiting distribution. Using simulations, Kwiatkowski et al. (1992) obtained almost identical quantile values.

Table 3: Quantile values of the limiting c.d.f. of the KPSS statistic.

\begin{tabular}{|c|c|c|c|c|c|c|}
\hline $\mathbf{P}\left(U_{\text {KPSS }} \leq x\right)$ & 0.75 & 0.80 & 0.85 & 0.90 & 0.95 & 0.99 \\
\hline$x$ & 0.20939 & 0.24124 & 0.28406 & 0.34730 & 0.46136 & 0.74346 \\
\hline
\end{tabular}

These quantiles can also be obtained by using the serial representation of $U_{K P S S}$ in Rosenblatt (1952):

$$
U_{K P S S}=\frac{1}{\pi^{2}} \sum_{i=1}^{\infty} \frac{x_{i}^{2}}{i^{2}}
$$

where $\left\{x_{i}\right\}$ is a sequence of independent standard normal random variables. Applying the well known infinite series expansions (see, for example, Bronshtein and Semendyayev (2000)), we can easily get the values of $\mathbf{E}\left(U_{K P S S}\right)$ and $\operatorname{Var}\left(U_{K P S S}\right)$. Indeed, under the null hypothesis,

$$
\mathbf{E}\left(U_{K P S S}\right)=\frac{1}{\pi^{2}} \sum_{i=1}^{\infty} \frac{1}{i^{2}}=\frac{1}{\pi^{2}} \cdot \frac{\pi^{2}}{6}=\frac{1}{6} .
$$

Next, note that if $x_{i}$ s are i.i.d. standard normal random variables, then $x_{i}^{2}$ s are i.i.d. $\chi^{2}$ random variables with mean 1 and variance 2 . Also, by independence the variance 
of a sum becomes just the sum of the variances. Therefore

$$
\begin{aligned}
\operatorname{Var}\left(U_{K P S S}\right) & =\operatorname{Var}\left(\frac{1}{\pi^{2}} \sum_{\imath=1}^{\infty} \frac{x_{\imath}^{2}}{i^{2}}\right)=\frac{1}{\pi^{4}} \operatorname{Var}\left(\sum_{\imath=1}^{\infty} \frac{x_{\imath}^{2}}{i^{2}}\right) \\
& =\frac{1}{\pi^{4}} \sum_{i=1}^{\infty} \frac{1}{i^{4}} \operatorname{Var}\left(x_{\imath}^{2}\right)=\frac{2}{\pi^{4}} \sum_{\imath=1}^{\infty} \frac{1}{i^{4}}=\frac{2}{\pi^{4}} \cdot \frac{\pi^{4}}{90}=\frac{1}{45}
\end{aligned}
$$

Under the alternative hypothesis of long memory, with extra condition that the cumulants satisfy

$$
\sup _{h} \sum_{r, s=-n}^{n}|k(h, r, s)|=O\left(n^{2 d}\right),
$$

the KPSS statistic is weakly convergent:

$$
\left(\frac{q}{n}\right)^{2 d} K_{n}(q) \stackrel{D}{\longrightarrow} Z_{K P S S}
$$

where $Z_{K P S S}=\int_{0}^{1}\left(W_{1 / 2+d}^{0}(t)\right)^{2} d t$ and $W_{1 / 2+d}^{0}(t)$ is a fractional Brownian bridge.

In the next section, we shall discuss a centered version of the KPSS statistic, called a $\mathrm{V} / \mathrm{S}$ statistic. In the limit, the behavior of both statistics is very similar. Therefore, we do not discuss the asymptotic behavior of a KPSS statistic in detail. Instead, in the next section, we focus our attention on the asymptotic behavior of a $\mathrm{V} / \mathrm{S}$ statistic.

$\mathrm{R}$ code for computing KPSS statistic is given in the Appendix. 


\subsection{V/S Statistic}

\subsubsection{Definition of $\mathrm{V} / \mathrm{S}$ Statistic}

Giraitis, Kokoszka, Leipus (2000) proposed a centered version of the KPSS statistic and called it a rescaled variance statistic, or $\mathrm{V} / \mathrm{S}$ statistic. Let

$$
V_{n}=\frac{1}{n^{2}}\left[\sum_{k=1}^{n}\left(\sum_{j=1}^{k}\left(X_{j}-\bar{X}\right)\right)^{2}-\frac{1}{n}\left(\sum_{k=1}^{n} \sum_{j=1}^{k}\left(X_{j}-\bar{X}\right)\right)^{2}\right]
$$

Then the $\mathrm{V} / \mathrm{S}$ statistic is given by

$$
T_{n}(d)=\left(\frac{q}{n}\right)^{2 d} \frac{V_{n}}{\hat{\sigma}_{n, q}^{2}},
$$

where $\hat{\sigma}_{n, q}^{2}$ is the same as in the definition of $R / S_{\text {mod }}$ and $K_{n}(q)$, and $q \rightarrow \infty$ as $n \rightarrow \infty$ in such a way that $q / n \rightarrow 0$.

A nice property of $T_{n}(d)$ is that it is robust against minor trends. Under certain conditions discussed later, for all $-1 / 2<d<1 / 2$, the statistic $T_{n}(d)$ converges weakly to a limiting distribution. All these properties make the $\mathrm{V} / \mathrm{S}$ statistic very useful in testing for long memory for a wide range of alternatives.

\subsubsection{Asymptotic Behavior of V/S Statistic}

We shall consider the asymptotic behaviour of $T_{n}(d)$ in connection with the test for testing the stationarity null hypothesis against nonstationary alternatives suggested by Giraitis et al. (2006). Initially, they tested the null hypothesis

$$
H_{0}: X_{t}=\mu+\xi_{t},
$$


where $\mu$ is a real number, and $\left\{\xi_{t}\right\}_{t \in \mathbb{Z}}$ is a stationary sequence with zero mean and covariance $\gamma_{t} \in G(d)$. The notation $G(d)$ is used to denote the family of processes with different covariance structures:

(a) Short memory - covariances $\gamma_{\jmath}$ are absolutely summable:

$$
\sum_{\jmath=-\infty}^{\infty}\left|\gamma_{\jmath}\right|<\infty, \quad \sum_{\jmath=-\infty}^{\infty} \gamma_{\jmath} \neq 0, \quad d=0
$$

(b) Long memory - covariances $\gamma_{\jmath}$ are slowly decaying: $\gamma_{\jmath} \sim c j^{2 d-1}, \quad$ as $k \rightarrow \infty, \quad$ where $c \neq 0 \quad$ and $\quad 0<d<1 / 2$;

(c) Negative memory - covariances $\gamma_{\jmath}$ are summing to zero:

$$
\gamma_{\jmath} \sim c j^{2 d-1}, \quad \sum_{\jmath=-\infty}^{\infty} \gamma_{\jmath}=0, \quad \text { where } c \neq 0 \quad \text { and } \quad-1 / 2<d<0
$$

This way, the null hypothesis covers all stationary type time series: short, long, and negative memory. Giraitis et al. (2006) also introduced three assumptions on the process under $H_{0}$.

Assumption 1 The finite-dimensional distrubutıons of $X_{n}(t)=n^{-1 / 2-d} \sum_{j=1}^{[n t]+1} \xi_{\jmath}$, $0 \leq t \leq 1$, converge to those of $\sigma_{d} W_{\frac{1}{2}+d}(t)$, where $W_{\frac{1}{2}+d}(t)$ is a fBm and

$$
\sigma_{d}^{2}=\left\{\begin{array}{cc}
\sum_{\jmath=-\infty}^{\infty} \gamma_{\jmath}, & \text { if } d=0 \\
\frac{c}{d(2 d+1)}, & \text { if } d \neq 0
\end{array}\right.
$$

where $c$ us a constant from the cases (b) and (c) above. (For details on convergence, see Section 2.3.2.)

Assumption 2 As $n \rightarrow \infty$,

$$
\frac{\sum_{\imath, \jmath=1}^{q} \hat{\gamma}_{|\imath-\jmath|}}{\sum_{\imath, \jmath=1}^{q} \gamma_{|\imath-\jmath|}} \stackrel{P}{\longrightarrow} 1,
$$


where $\hat{\gamma}_{|i-j|}$ is the sample autocovariance of $\left\{\xi_{t}\right\}$ at lag $t=|i-j|$ and $q=q(n)$ is a bandwidth parameter, dependent on the sample size $n$.

We need this condition to ensure that $q^{-2 d} \hat{\sigma}_{n, q}^{2} \stackrel{P}{\longrightarrow} \sigma_{d}^{2}$, as $n \rightarrow \infty$. Next, assume that $q=O\left(n^{\frac{1}{2}}\right)$.

Assumption 3 Under the assumption of stationarity, an estimator $\hat{d}$ of $d$ is consistent at least at the logarithmic rate, i.e., $\hat{d}-d=o_{P}(1 / \log n)$, and takes values in $\left[-a_{1}, a_{2}\right] \in(-1 / 2,1 / 2)$.

Any estimator $\hat{d}$ of $d$ with the above mentioned properties will work for the statistic $T_{n}(d)$. For example, Giraitis et al. (2006) used a Whittle estimator (see Section 3.4 about this estimator).

We now discuss the use of $\mathrm{V} / \mathrm{S}$ statistic for testing short memory versus long memory in the presence of a weak trend. Recall that the $\mathrm{V} / \mathrm{S}$ statistic is given by $T_{n}(d)=\left(\frac{q}{n}\right)^{2 d} \frac{V_{n}}{\hat{\sigma}_{n, q}^{2}}$. Although $T_{n}(d)$ allows for testing stationarity versus nonstationarity, in what follows, we confine ourselves to the use of $T_{n}(d)$ for testing short memory versus long memory in the presence of a weak trend.

The following theorem is due to Giraitis et al. (2006). We go over its proof in details filling in some skipped parts.

Theorem 4.1. i) Under the null hypothesis of short memory and Assumptions 1-3, the statistic $T_{n}(\hat{d})$ converges weakly to $Z_{0}$, where

$$
Z_{0}=\int_{0}^{1}\left(W^{0}(t)-\int_{0}^{1} W^{0}(u) d u\right)^{2} d t
$$

with $W^{0}(t)$ being a Brownian bridge. 
ii) Under the alternative hypothesis of long memory and Assumptions 1-3, the statistic $T_{n}(\hat{d})$ converges weakly to $Z_{d}$, where

$$
Z_{d}=\int_{0}^{1}\left(W_{1 / 2+d}^{0}(t)-\int_{0}^{1} W_{1 / 2+d}^{0}(u) d u\right)^{2} d t
$$

with $W_{1 / 2+d}^{0}(t)$ being a fractional Brownian bridge.

Comments prior to the proof. As the statistic $T_{n}(d)$ is robust against weak trends, Giraitis et al. (2006) relaxed the null hypothesis in (4.3) to incorporate weak deterministic trend without changing the asymptotic properties. The modified null hypothesis has the form

$$
X_{t}=\mu+g_{n}(t)+\xi_{t}
$$

where $g_{n}(t)$ is a deterministic trend satisfying $\sum_{k=1}^{n} g_{n}^{2}(k)=o\left(n^{2 d}\right)$ as $n \rightarrow \infty$, and $\left\{\xi_{t}\right\}$ is a zero mean covariance stationary sequence with $\gamma_{k} \in G(d), d \in\left[-a_{1}, a_{2}\right] \in$ $(-1 / 2,1 / 2)$. Asymptotic properties of $T_{n}(d)$ under hypothesis (4.5) will be discussed later in this section.

Using Monte Carlo simulation with $n=10,000$ and $q=n^{1 / 3}$ applied to the FARIMA $(0, d, 0)$ model, Giraitis et al. (2006) obtained quantiles of the limiting distribution of $Z_{d}$. These quantiles are presented in Table 4.

Table 4: Quantile values of the limiting c.d.f. of the $V / S$ statistic.

\begin{tabular}{cccccccccc}
\hline$d$ & -0.4 & -0.3 & -0.2 & -0.1 & 0 & 0.1 & 0.2 & 0.3 & 0.4 \\
\hline$c_{5 \%}(d)$ & 0.597 & 0.457 & 0.349 & 0.262 & 0.190 & 0.130 & 0.083 & 0.049 & 0.022 \\
$c_{10 \%}(d)$ & 0.524 & 0.393 & 0.292 & 0.213 & 0.153 & 0.105 & 0.067 & 0.039 & 0.016 \\
\hline
\end{tabular}


Using least squares fitting, Giraitis et al. (2006) also derived formulas for the $5 \%$ and $10 \%$ quantiles:

$$
\begin{aligned}
& c_{5 \%}(d)=-1.98 d^{5}+0.73 d^{4}-0.05 d^{3}+0.63 d^{2}-0.66 d+0.19 \\
& c_{10 \%}(d)=-0.27 d^{5}+0.48 d^{4}-0.55 d^{3}+0.66 d^{2}-0.53 d+0.153 .
\end{aligned}
$$

For $d=0$ the distribution function of $Z_{d}$ was explicitly derived by Watson (1961):

$$
F_{Z_{0}(x)}=\mathbf{P}\left(Z_{0} \leq x\right)=1+2 \sum_{\jmath=1}^{\infty}(-1)^{\jmath} e^{-2 \jmath^{2} \pi^{2} x}
$$

Also, $Z_{0}$ has a series representation (see Giraitis et al. (2003))

$$
Z_{0}=\frac{1}{4 \pi^{2}} \sum_{\jmath=1}^{\infty} \frac{z_{2 j-1}^{2}+z_{2 \jmath}^{2}}{j^{2}}
$$

where $\left\{z_{\jmath}\right\}$ are independent standard normal random variables. Based on (4.7) one can easily compute the expectation and variance of $Z_{0}$ :

$$
\mathbf{E}\left(Z_{0}\right)=\frac{1}{12} \quad \text { and } \quad \operatorname{Var}\left(Z_{0}\right)=\frac{1}{360} .
$$

We have recalculated the quantile values of the distribution of $Z_{0}$ by using representation (4.6) and truncating the sum at $j=100,000$. The obtained values of the $5 \%$ and $10 \%$ quantiles are almost the same as those in Table $4:$ 
Table 5: Updated quantile values of the limiting c.d.f. of the $V / S$ statistic for $d=0$.

\begin{tabular}{|c|c|c|c|}
\hline$\alpha$ & $1 \%$ & $5 \%$ & $10 \%$ \\
\hline$c_{\alpha}(d)$ & 0.269 & 0.187 & 0.152 \\
\hline
\end{tabular}

We shall now consider in more detail the asymptotic behaviour of

$$
T_{n}(d)=\frac{V_{n}}{n^{2 d}} \frac{q^{2 d}}{\hat{\sigma}_{n, q}^{2}}
$$

under the validity of hypothesis (4.5). First, we explain informally a general idea of what we will be proving.

It can be shown that under the modified null hypothesis (4.5)

$$
\frac{V_{n}}{n^{2 d}} \stackrel{D}{\longrightarrow} V_{\infty}
$$

where

$$
V_{\infty}=\int_{0}^{1}\left(\sigma_{d}\left(W_{\frac{1}{2}+d}(t)-t W_{\frac{1}{2}+d}(1)\right)-\int_{0}^{1} \sigma_{d}\left(W_{\frac{1}{2}+d}(u)-u W_{\frac{1}{2}+d}(1)\right) d u\right)^{2} d t
$$

The limiting random variable $V_{\infty}$ equals in distribution to $\sigma_{d}^{2} Z_{d}$, where $Z_{d}$ is the same as in Theorem 4.1 and the constant $\sigma_{d}^{2}$ is defined by (4.4). Next, if we check that

$$
\frac{\hat{\sigma}_{n, q}^{2}}{q^{2 d}} \stackrel{P}{\longrightarrow} \sigma_{d}^{2}
$$

then by Slutsky's lemma, the convergence results in Theorem 4.1 will follow. Now we give a detailed proof of Theorem 4.1 . 
Proof of the theorem 4.1:

Part I. Numerator's convergence. We start by examining the limiting behaviour of $n^{-2 d} V_{n}$. Put $S_{k}=\sum_{\jmath=1}^{k}\left(X_{\jmath}-\bar{X}\right)$ and $\bar{X}=\frac{1}{n} \sum_{j=1}^{n} X_{\jmath}$. Then we have

$$
\begin{aligned}
V_{n} & =\frac{1}{n^{2}}\left[\sum_{k=1}^{n} S_{k}^{2}-\frac{1}{n}\left(\sum_{k=1}^{n} S_{k}\right)^{2}\right]=\frac{1}{n^{2}} \sum_{k=1}^{n}\left(S_{k}-n^{-1} \sum_{\jmath=1}^{n} S_{\jmath}\right)^{2} \\
& =n^{-1} \int_{0}^{1}\left(S_{[n t]+1}-\int_{0}^{1} S_{[n u]+1} d u\right)^{2} d t .
\end{aligned}
$$

Indeed, equality (4.8) is obvious:

$$
\begin{gathered}
\frac{1}{n^{2}} \sum_{k=1}^{n}\left(S_{k}-n^{-1} \sum_{\jmath=1}^{n} S_{\jmath}\right)^{2}=\frac{1}{n^{2}} \sum_{k=1}^{n}\left(S_{k}-\bar{S}\right)^{2}=\frac{1}{n^{2}} \sum_{k=1}^{n}\left(S_{k}-2 S_{k} \bar{S}+\bar{S}^{2}\right) \\
=\frac{1}{n^{2}} \sum_{k=1}^{n} S_{k}^{2}-\frac{2 \bar{S}}{n^{2}} \sum_{k=1}^{n} S_{k}+\frac{\bar{S}^{2}}{n}=\frac{1}{n^{2}} \sum_{k=1}^{n} S_{k}^{2}-\frac{1}{n} \bar{S}^{2}=\frac{1}{n^{2}}\left(\sum_{k=1}^{n} S_{k}^{2}-\frac{1}{n}\left(\sum_{k=1}^{n} S_{k}\right)^{2}\right) .
\end{gathered}
$$

In order to justify equality (4.9), note that

$$
\frac{1}{n} \sum_{\jmath=1}^{n} S_{\jmath}=\int_{0}^{1} S_{[n u]+1} d u
$$

where $S_{[n u]+1}=S_{\jmath}$ provided $j-1 \leq n u<j$ and $0 \leq u \leq 1$. Consequently, $u$ varies in the interval $[(j-1) / n, j / n]$ and $d u=1 / n$. Therefore, we can write $V_{n}$ in the form

$$
V_{n}=n^{-2} \sum_{k=1}^{n}\left(S_{k}-\int_{0}^{1} S_{[n u]+1} d u\right)^{2}
$$


Denoting $S_{k}=S_{[n t]}+1$ for $k-1 \leq n t<k$ and $0 \leq t \leq 1$, we get

$$
V_{n}=n^{-1} \int_{0}^{1}\left(S_{[n t]+1}-\left(\int_{0}^{1} S_{[n u]+1} d u\right)\right)^{2} d t
$$

and

$$
n^{-2 d} V_{n}=\int_{0}^{1}\left(n^{-1 / 2-d} S_{[n t]+1}-\int_{0}^{1} n^{1 / 2-d} S_{[n u]+1} d u\right)^{2} d t
$$

Define the process

$$
Y_{n}(t)=n^{-1 / 2-d} \sum_{j=1}^{[n t]+1}\left(X_{j}-\mu\right), \quad 0 \leq t \leq 1
$$

Using (4.10), we can write

$$
n^{-1 / 2-d} S_{[n t]+1}=Y_{n}(t)-t Y_{n}(1)+r_{n}(t)
$$

where $r_{n}(t)$ is negligible, that is, for any $t \in[0,1], r_{n}(t) \stackrel{P}{\longrightarrow} 0$ as $n \rightarrow \infty$.

Indeed, consider the remainder term

$$
r_{n}(t)=n^{-1 / 2-d} S_{[n t]+1}-Y_{n}(t)+t Y_{n}(1)
$$

and note that for $k-1 \leq n t<k,[n t]+1=k$. To simplify calculations, let $\mu=0$. Then, using the facts that $Y_{n}(1) / n \stackrel{P}{\longrightarrow} 0$ and $n^{-1 / 2-d} X_{n+1}=Y_{n}(1)-Y_{n-1}(1)$, we 
have

$$
\begin{gathered}
r_{n}(t)=n^{-1 / 2-d}\left[\sum_{\jmath=1}^{k} X_{\jmath}-k \bar{X}-\sum_{\jmath=1}^{k} X_{\jmath}+t \sum_{\jmath=1}^{n+1} X_{\jmath}\right] \\
\leq\left|n^{-1 / 2-d}\left(-k \bar{X}+t \sum_{\jmath=1}^{n+1} X_{\jmath}\right)\right|=\left|n^{-1 / 2-d}\left(-\frac{k}{n} \sum_{\jmath=1}^{n+1} X_{\jmath}+t \sum_{\jmath=1}^{n+1} X_{\jmath}+\frac{k}{n} X_{n+1}\right)\right| \\
=\left|\left(t-\frac{k}{n}\right) n^{-1 / 2-d} \sum_{\jmath=1}^{n+1} X_{\jmath}+n^{-1 / 2-d} \frac{k}{n} X_{n+1}\right| \leq \frac{1}{n}\left(\left|Y_{n}(1)\right|+n^{-1 / 2-d}\left|X_{n+1}\right|\right)=o_{P}(1) .
\end{gathered}
$$

Therefore we can write

$$
\begin{aligned}
n^{-2 d} V_{n} & =\int_{0}^{1}\left(Y_{n}(t)-t Y_{n}(1)-\int_{0}^{1}\left(Y_{n}(u)-u Y_{n}(1)\right) d u+o_{P}(1)\right)^{2} d t \\
& =V_{n}^{\prime}+\left|V_{n}^{\prime}\right|^{1 / 2} o_{P}(1)+o_{P}(1),
\end{aligned}
$$

where

$$
V_{n}^{\prime}=\int_{0}^{1}\left(Y_{n}(t)-t Y_{n}(1)-\int_{0}^{1}\left(Y_{n}(u)-u Y_{n}(1)\right) d u\right)^{2} d t
$$

Now, we need to check that conditions (i)-(iii) of Theorem 2.3 are satisfied. Recall that under the null hypothesis, $X_{t}=\mu+g_{n}(t)+\xi_{t}$, where $\sum_{j=1}^{n} g_{n}^{2}(t)=o\left(n^{2 d}\right)$. From this

$$
Y_{n}(t)=n^{-1 / 2-d} \sum_{\jmath=1}^{[n t]+1} \xi_{\jmath}+n^{-1 / 2-d} \sum_{\jmath=1}^{[n t]+1} g_{n}(t)
$$


Then by the Schwartz inequality,

$$
\begin{aligned}
n^{-1 / 2-d}\left|\sum_{j=1}^{[n t]+1} 1 \cdot g_{n}(t)\right| & \leq n^{-1 / 2-d}\left(\sum_{j=1}^{[n t]+1} 1^{2}\right)^{\frac{1}{2}}\left(\sum_{t=1}^{[n t]+1} g_{n}^{2}(t)\right)^{\frac{1}{2}} \\
& \leq n^{-1 / 2-d} \sqrt{n+1}\left(\sum_{j=1}^{[n t]+1} g_{n}^{2}(t)\right)^{\frac{1}{2}}=n^{-d} o\left(n^{d}\right)=o(1)
\end{aligned}
$$

From here, we get

$$
Y_{n}(t)=n^{-1 / 2-d} \sum_{j=1}^{[n t]+1} \xi_{j}+o_{P}(1)
$$

Note that we write $o_{P}(1)$ instead of $o(1)$, since $Y_{n}(t)$ is a random variable.

Now observe that Assumption 1 implies f.d.d. convergence of $n^{1 / 2-d} \sum_{j=1}^{[n t]+1} \xi_{j}$ to $Y_{\infty}(t)=\sigma_{d} W_{1 / 2+d}(t)$. Hence, for the process $Y_{n}(t)$, condition (i) of Theorem 2.3 is satisfied with $Y_{\infty}(t)$ in the limit. It remains to note that by the fact

$$
\mathbf{E}\left[n^{-1 / 2-d} \sum_{j=1}^{[n t]+1} \xi_{j}\right]^{2} \rightarrow t^{1+2 d} \sigma_{d}^{2}=\mathbf{E} X^{2}(t)
$$

conditions (ii) and (iii) of Theorem 2.3 are also satisfied.

Therefore by the Corollary of Theorem 2.3

$$
V_{n}^{\prime} \stackrel{D}{\longrightarrow} V_{\infty}
$$

where

$$
V_{\infty}=\int_{0}^{1}\left(\sigma_{d}\left(W_{1 / 2+d}(t)-t W_{1 / 2+d}(1)\right)-\int_{0}^{1} \sigma_{d}\left(W_{1 / 2+d}(u)-u W_{1 / 2+d}(1)\right) d u\right)^{2} d t
$$


Thus

$$
n^{-2 d} V_{n} \stackrel{D}{\longrightarrow} \sigma_{d}^{2} Z_{d}
$$

where

$$
\sigma_{d}^{2} Z_{d}=V_{\infty}=\int_{0}^{1}\left(\sigma_{d} W_{1 / 2+d}^{0}(t)-\int_{0}^{1} \sigma_{d} W_{1 / 2+d}^{0}(u) d u\right)^{2} d t
$$

and $W_{1 / 2+d}^{0}(t)=W_{1 / 2+d}(t)-t W_{1 / 2+d}(1)$ is a fractional Brownian bridge on the unit interval.

Part II. Denominator's convergence. Next consider the statistic $q^{-2 d} \hat{\sigma}_{n, q}^{2}$, where

$$
\hat{\sigma}_{n, q}^{2}=\frac{1}{q} \sum_{\imath, \jmath=1}^{q} \hat{\gamma}_{|\imath-\jmath|, X}, \quad \hat{\gamma}_{|\imath-\jmath|, X}=\frac{1}{n} \sum_{t=1}^{n-|\imath-\jmath|}\left(X_{t}-\bar{X}\right)\left(X_{t+|\imath-\jmath|}-\bar{X}\right)
$$

and recall that under $H_{0}$

$$
X_{t}=\mu+g_{n}(t)+\xi_{t}
$$

If we again assume for simplicity that $\mu=0$, then $\hat{\gamma}_{|\imath-\jmath|, X}$ becomes

$$
\begin{gathered}
\hat{\gamma}_{|\imath-\jmath|, X}=\frac{1}{n} \sum_{t=1}^{n-|\imath-\jmath|}\left(g_{n}(t)-(\bar{g}+\bar{\xi})+\xi_{t}\right)\left(g_{n}(t+|i-j|)-(\bar{g}+\bar{\xi})+\xi_{t+|\imath-\jmath|}\right) \\
=\frac{1}{n} \sum_{t=1}^{n-|\imath-\jmath|}\left(g_{n}(t)-\bar{g}+\xi_{t}-\bar{\xi}\right)\left(g_{n}(t+|i-j|)-\bar{g}+\xi_{t+|\imath-\jmath|}-\bar{\xi}\right) \\
=\frac{1}{n} \sum_{t=1}^{n-|\imath-\jmath|}\left(g_{n}(t)-\bar{g}\right)\left(g_{n}(t+|i-j|)-\bar{g}\right)+\frac{1}{n} \sum_{t=1}^{n-|\imath-\jmath|}\left(\xi_{t}-\bar{\xi}\right)\left(\xi_{t+|\imath-\jmath|}-\bar{\xi}\right) \\
+\frac{1}{n} \sum_{t=1}^{n-|\imath-\jmath|}\left(g_{n}(t)-\bar{g}\right)\left(\xi_{t+|\imath-\jmath|}-\bar{\xi}\right)+\frac{1}{n} \sum_{t=1}^{n-|\imath-\jmath|}\left(\xi_{t}-\bar{\xi}\right)\left(g_{n}(t+|i-j|)-\bar{g}\right) .
\end{gathered}
$$


The summands in line (4.11) are just $\hat{\gamma}_{|\imath-\jmath|, g}$ and $\hat{\gamma}_{|2-\jmath|, \xi}$. Therefore if we denote

$$
\hat{\sigma}_{n, g}^{2}=\frac{1}{q} \sum_{i, \jmath=1}^{q} \hat{\gamma}_{|2-\jmath|, g} \quad \text { and } \quad \hat{\sigma}_{n, \xi}^{2}=\frac{1}{q} \sum_{\imath, \jmath=1}^{q} \hat{\gamma}_{|2-\jmath|, \xi}
$$

we can write

$$
\hat{\sigma}_{n, q}^{2}=\hat{\sigma}_{n, g}^{2}+\hat{\sigma}_{n, \xi}^{2}+r_{n}
$$

where $r_{n}$ corresponds to line (4.12):

$r_{n}=\frac{1}{q} \sum_{\imath, \jmath=1}^{q}\left(\frac{1}{n} \sum_{t=1}^{n-|\imath-\jmath|}\left(g_{n}(t)-\bar{g}\right)\left(\xi_{t+|\imath-\jmath|}-\bar{\xi}\right)+\frac{1}{n} \sum_{t=1}^{n-|\imath-\jmath|}\left(\xi_{t}-\bar{\xi}\right)\left(g_{n}(t+|\imath-j|)-\bar{g}\right)\right)$.

Therefore

$$
q^{-2 d} \hat{\sigma}_{n, q}^{2}=q^{-2 d} \hat{\sigma}_{n, g}^{2}+q^{-2 d} \hat{\sigma}_{n, \xi}^{2}+q^{-2 d} r_{n}
$$

Each term on the right-hand side will be analyzed separately. First, consider $q^{-2 d} \hat{\sigma}_{n, g}^{2}$ :

$$
\left.q^{-2 d} \hat{\sigma}_{n, g}^{2}=q^{-1-2 d} \sum_{i, \jmath=1}^{q} \frac{1}{n} \sum_{t=1}^{n-|z-\jmath|}\left(g_{n}(t)-\bar{g}\right)\left(g_{n}(t+|i-\jmath|)-\bar{g}\right)\right) .
$$


Applying the Schwartz inequality and using the assumption that $\sum_{t=1}^{n}\left(g_{n}(t)\right)^{2}=o\left(n^{2 d}\right)$, we get

$$
\begin{aligned}
q^{-2 d} \hat{\sigma}_{n, g}^{2} & \leq q^{-1-2 d} \sum_{i, j=1}^{q} \frac{1}{n}\left(\sum_{t=1}^{n}\left(g_{n}(t)-\bar{g}\right)^{2}\right)^{\frac{1}{2}}\left(\sum_{t=1}^{n}\left(g_{n}(t)-\bar{g}\right)^{2}\right)^{\frac{1}{2}} \\
& =q^{-2 d} \frac{q}{n} \sum_{t=1}^{n}\left(g_{n}(t)-\bar{g}\right)^{2}=\frac{q^{1-2 d}}{n} \sum_{t=1}^{n}\left(g_{n}(t)-\bar{g}\right)^{2} \\
& \leq \frac{q^{1-2 d}}{n} \sum_{t=1}^{n}\left(g_{n}(t)\right)^{2}=\frac{q^{1-2 d}}{n} o\left(n^{2 d}\right)=o\left(\left(\frac{q}{n}\right)^{1-2 d}\right) .
\end{aligned}
$$

Next, consider the term $q^{-2 d} r_{n}$. Again, we shall use the Schwartz inequality and the fact that $\sum_{t=1}^{n} \xi_{t}^{2}=O_{P}(n)$, which is a consequence of the Markov inequality:

$$
\mathbf{P}\left(\frac{1}{n} \sum_{t=1}^{n} \xi_{t}^{2} \geq x\right) \leq \frac{\mathbf{E}\left(\frac{1}{n} \sum_{t=1}^{n} \xi_{t}^{2}\right)}{x}=\frac{\mathbf{E}\left(\xi_{1}^{2}\right)}{x} \rightarrow 0, \quad \text { as } x \rightarrow \infty .
$$

We have

$$
\begin{aligned}
q^{-2 d} r_{n} & \leq q^{-1-2 d} \sum_{i, j=1}^{q} \frac{2}{n}\left(\sum_{t=1}^{n}\left(g_{n}(t)-\bar{g}\right)^{2}\right)^{\frac{1}{2}}\left(\sum_{t=1}^{n}\left(\xi_{t}-\bar{\xi}\right)^{2}\right)^{\frac{1}{2}} \\
& =2 q^{1-2 d}\left(\frac{1}{n} \sum_{t=1}^{n}\left(g_{n}(t)-\bar{g}\right)^{2}\right)^{\frac{1}{2}}\left(\frac{1}{n} \sum_{t=1}^{n}\left(\xi_{t}-\bar{\xi}\right)^{2}\right)^{\frac{1}{2}} \\
& \leq q^{1-2 d}\left(\frac{1}{n} \sum_{t=1}^{n}\left(g_{n}(t)\right)^{2}\right)^{\frac{1}{2}}\left(\frac{1}{n} \sum_{t=1}^{n} \xi_{t}^{2}\right)^{\frac{1}{2}} \\
& =q^{1-2 d} \cdot o\left(n^{-1+2 d}\right)^{\frac{1}{2}} \cdot O_{P}(1)=o_{P}\left(\left(\frac{q^{2}}{n}\right)^{\frac{1}{2}-d}\right) .
\end{aligned}
$$

From our computations, noting that $q=O\left(n^{\frac{1}{2}}\right)$ as $n \rightarrow \infty$, we get that both $q^{-2 d} \hat{\sigma}_{n, g}^{2}$ and $q^{-2 d} r_{n}$ go to zero in probability. 
Finally, consider the term $q^{-2 d} \hat{\sigma}_{n, \xi}^{2}$, which gives the main contribution to the sum in (4.13). Let us show that $n^{-1-2 d} \sum_{\imath, j=1}^{q} \gamma_{|\imath-\jmath|, \xi} \rightarrow \sigma_{d}^{2}$, as $n \rightarrow \infty$. To do this, recall that for a long memory time series, covariance function is of the form $\gamma(h)=c h^{2 d-1}$. Therefore, using the convention that $|\imath-i|^{2 d-1}=1$, we have

$$
\begin{gathered}
n^{-1-2 d} \sum_{i, \jmath=1}^{n} \gamma_{|\imath-\jmath|, \xi}=n^{-1-2 d} c \sum_{i, \jmath=1}^{n}|\imath-j|^{2 d-1}=c n^{-1-2 d} \sum_{\imath=1}^{n}\left(\sum_{\jmath=1}^{n}|\imath-j|^{2 d-1}\right) \\
=c \sum_{\imath=1}^{n} n^{-1-2 d} \sum_{\jmath=1}^{n}\left(\left|\frac{i}{n}-\frac{\jmath}{n}\right|^{2 d-1} \cdot n^{-1+2 d}\right) \\
=c n^{-1} \sum_{i=1}^{n}\left(\sum_{\jmath=1}^{n}\left|\frac{i}{n}-\frac{j}{n}\right|^{2 d-1} \cdot \frac{1}{n}\right) \sim c n^{-1} \sum_{i=1}^{n} \int_{0}^{1}\left|\frac{i}{n}-x\right|^{2 d-1} d x \\
=c \sum_{i=1}^{n}\left(\int_{0}^{1}\left|\frac{i}{n}-x\right|^{2 d-1} d x\right) \frac{1}{n} \sim c \int_{0}^{1}\left(\int_{0}^{1}|y-x|^{2 d-1} d x\right) d y \\
=\frac{c}{d(2 d+1)}=\sigma_{d}^{2} .
\end{gathered}
$$

Using this result and Assumption 2 of Theorem 4.1, according to which

$$
\frac{\sum_{\imath, \jmath=1}^{q} \hat{\gamma}_{|2-\jmath|, \xi}}{\sum_{\imath, \jmath=1}^{q} \gamma_{|2-\jmath|, \xi}} \stackrel{P}{\longrightarrow} 1,
$$

we have

$$
q^{-2 d} \hat{\sigma}_{n, \xi}^{2}=\frac{\frac{1}{q} \sum_{i, \jmath=1}^{q} \hat{\gamma}_{|\imath-\jmath|, \xi}}{q^{2 d}}=q^{-1-2 d} \frac{\sum_{i, \jmath=1}^{q} \hat{\gamma}_{|2-\jmath|, \xi}}{\sum_{\imath, \jmath=1}^{q} \gamma_{|\imath-\jmath|, \xi}}\left(\sum_{\imath, \jmath=1}^{q} \gamma_{|\imath-\jmath|, \xi}\right) \stackrel{P}{\longrightarrow} \sigma_{d}^{2}
$$


From this, recalling (4.13) and combining the obtained results for $q^{-2 d} \hat{\sigma}_{n, 9}^{2}$ and $q^{-2 d} r_{n}$, we conclude that

$$
q^{-2 d} \hat{\sigma}_{n, q}^{2} \stackrel{P}{\longrightarrow} \sigma_{d}^{2}
$$

Return to the statistic $T_{n}(d)$. It has been shown above that

$$
n^{-2 d} V_{n} \stackrel{D}{\longrightarrow} \sigma_{d}^{2} Z_{d} \quad \text { and } \quad q^{-2 d} \hat{\sigma}_{n, q}^{2} \stackrel{P}{\longrightarrow} \sigma_{d}^{2}
$$

where $\sigma_{d}^{2}$ is a constant. Therefore, by Slutsky's lemma,

$$
\text { (a) } T_{n}(d) \stackrel{D}{\longrightarrow} Z_{0} \text { for } d=0 ; \quad \text { (b) } T_{n}(d) \stackrel{D}{\longrightarrow} Z_{d} \text { for } d>0 \text {, }
$$

which completes the proof of Theorem 4.1.

$\mathrm{R}$ code for computing the value of $\mathrm{V} / \mathrm{S}$ statistic is given in the Appendix.

In the proof of Theorem 4.1, the fact that

$$
\frac{1}{n} \sum_{t=1}^{n} \xi_{t}^{2}=O_{P}(1)
$$

has been used. For linear processes, we may strengthen this asymptotic result. The following proposition holds true.

Proposition 4.1. Let $\left\{\xi_{t}\right\}$ be a linear process of the form $\xi_{t}=\sum_{\jmath=1}^{\infty} a_{\jmath} \varepsilon_{t-\jmath}$, where $\varepsilon_{t}$ are i.l.d. with $\mathbf{E}\left(\varepsilon_{t}\right)=0$ and $\operatorname{Var}\left(\varepsilon_{t}\right)=1$, and assume that $\gamma_{\jmath} \in G(d), d \in(-1 / 2,1 / 2)$. Then

$$
\frac{1}{n} \sum_{t=1}^{n} \xi_{t}^{2} \stackrel{P}{\rightarrow} \mathbf{E}\left(\xi_{1}^{2}\right)=\sum_{\jmath=1}^{\infty} a_{\jmath}^{2} .
$$


This follows from the fact that a strong linear process (i.e. when innovations are independent, and therefore ergodic) is ergodic, as a function of ergodic process.

\subsection{Increment Ratio Statistic}

The most recent addition to the family of $\mathrm{R} / \mathrm{S}$ type statistics is the so-called increment ratio (IR) statistic introduced by Surgailis et al. (2008). We shall give the definition of this new statistic and describe its asymptotic behavior without proof. The test based on IR statistic is very powerful. It can be used for testing the nonparametric hypothesis of $d$-integrated behavior of time series $\left\{X_{t}\right\}$ with $-1 / 2<d<3 / 2$. This range for $d$ covers both short memory $(d=0)$, stationary long-memory $(0<d<1 / 2)$, and unit roots $(d=1)$. The IR test, constructed to test the null hypothesis of short memory presence $(d=0)$ against stationary long-memory alternatives, shows the robustness against change in mean, slowly varying trends, and nonstationarities. For details, see Surgailis et al. (2008).

The IR statistic is defined by

$$
I R=\frac{1}{n-3 m} \sum_{k=0}^{n-3 m-1} \frac{\left|\sum_{t=k+1}^{k+m}\left(X_{t+m}-X_{t}\right)+\sum_{t=k+m+1}^{k+2 m}\left(X_{t+m}-X_{t}\right)\right|}{\left|\sum_{t=k+1}^{k+m}\left(X_{t+m}-X_{t}\right)\right|+\left|\sum_{t=k+m+1}^{k+2 m}\left(X_{t+m}-X_{t}\right)\right|}
$$

where $X_{1}, X_{2}, \ldots, X_{n}$ is a sample of size $n$, and $m$ is a bandwidth parameter.

The asymptotic behaviour of IR statistic under the null hypothesis of stationarity was established by Surgailis et al. (2008) who showed that if $\left\{X_{t}\right\}_{t \in \mathbb{Z}}$ is stationary, then

$$
I R \stackrel{P}{\longrightarrow} \Lambda(d), \quad \text { where } \quad \Lambda(d)=\mathbf{E}\left[\frac{\left|Z_{d}(0)+Z_{d}(1)\right|}{\left|Z_{d}(0)\right|+\left|Z_{d}(1)\right|}\right]
$$


and the vector $\left(Z_{d}(0), Z_{d}(1)\right)$ has Gaussian distribution with

$\mathbf{E}\left(Z_{d}(\cdot)\right)=0, \quad \operatorname{Var}\left(Z_{d}(\cdot)\right)=1, \quad \rho(d)=\operatorname{Cov}\left(Z_{d}(0), Z_{d}(1)\right)=\frac{-9^{d+0.5}+4^{d+1.5}-7}{2\left(4-4^{d+0.5}\right)}$

\subsection{Preliminary Test Estimator}

In this section, we first discuss a concept of preliminary test estimator (PTE). Then, by using this concept, we make an attempt to improve the performance of $\mathrm{V} / \mathrm{S}$ statistic.

The PTE was introduced by Bankroft in 1944. The estimator is used to estimate parameters in the suspected presence of some uncertain prior information about some of them. Before applying the concept of PTE to the problem of long memory detection, we shall discuss this concept informally. For formal definition, properties, and other details, see the monograph of Saleh (2006).

The idea behind PTE is as follows. Suppose there is an unknown vector of parameters $\boldsymbol{\theta}=\left(\theta_{1}, \theta_{2}\right) \in \Theta \subset \mathbb{R}^{2}$. We are interested in estimating $\theta_{2}$, and there is a suspicion that the actual value of $\theta_{1}$ is $a$. A preliminary test estimator helps to improve the estimation of $\theta_{2}$ by using the preliminary information about $\theta_{1}$. Two estimators are formed:

(a) an unrestricted estimator $\hat{\theta}_{2}=g\left(\hat{\theta}_{1}, \mathbf{X}\right)$, where $\mathbf{X}$ is a sample vector, which does not use the prior information about $\theta_{1}$.

(b) a restricted estimator $\tilde{\theta}_{2}=g(a, \mathbf{X})$, which uses the prior knowledge of $\theta_{1}$.

Intuitively, the estimator $\tilde{\theta}_{2}$ should be more precise than the estimator $\hat{\theta}_{2}$. However, there is a problem: the prior information is uncertain. This problem is solved by testing

$$
\mathbf{H}_{0}: \theta_{1}=a \text { vs. } \quad \mathbf{H}_{a}: \theta_{1} \neq a
$$


As a result of the test, we choose either $\tilde{\theta}_{2}$ or $\hat{\theta}_{2}$ based on whether we reject or accept $\mathbf{H}_{0}$. Often, for estimating the vector of parameters $\boldsymbol{\theta}$, the maximum likelihood estimation method is used.

In connection with the problem of detecting long memory, the concept of PTE needs to be corrected. The first problem that has to be dealt with is that long memory is determined by a single parameter $d$. The second problem is that the application of PTE must improve the quality of testing. We shall apply PTE, for example to the $\mathrm{V} / \mathrm{S}$ statistic $T_{n}(d)$. Before, the PTE method was used mostly for estimation.

Suppose we suspect that the data are short range dependent. In this case $d=0$ and the restricted statistic is $T_{n}(0)$. The unrestricted statistic $T_{n}(\hat{d})$ is formed by estimating $d$ by means of the maximum likelihood Whittle estimator discussed in Section 3.4. To eliminate the uncertainty in the prior information, we test

$$
\mathbf{H}_{0}: d=0 \quad \text { vs. } \quad \mathbf{H}_{a}: d \neq 0
$$

To run this test, we shall use the result of Robinson (1995) for a Whittle estimator. When applied to our case under $H_{0}: d=0$, this result yields

$$
\sqrt{m}(\hat{d}-0) \stackrel{D}{\longrightarrow} N\left(0, \frac{1}{4}\right)
$$

where $m$ is a window parameter. In the sequel, we take $m$ to be $m=n^{0.7}$.

The resulting statistic, obtained after the application of the PTE method to $T_{n}(d)$, will be denoted by $\left[T_{n}(d)\right]_{P T E}$. Then, if the Whittle estimate $\hat{d}$ falls into the acceptance region of the level $\alpha$ test, we will choose $\left[T_{n}(d)\right]_{P T E}=T_{n}(0)$. Otherwise, we will take $\left[T_{n}(d)\right]_{P T E}=T_{n}(\hat{d})$. 
Let us now formalize our idea. For this, define the events

$$
\begin{aligned}
& A_{n}=\left\{\mathbf{X}: 2 \sqrt{m}|\hat{d}(\mathbf{X})| \leq \Phi^{-1}\left(1-\frac{\alpha}{2}\right)\right\} \\
& A_{n}^{\prime}=\left\{\mathbf{X}: 2 \sqrt{m}|\hat{d}(\mathbf{X})|>\Phi^{-1}\left(1-\frac{\alpha}{2}\right)\right\}
\end{aligned}
$$

where $\mathbf{X}=\left(X_{1}, \ldots, X_{n}\right)$ is the data, $m=n^{0.7}$, and $\Phi^{-1}\left(1-\frac{\alpha}{2}\right)$ is the quantile of order $(1-\alpha / 2)$ of a standard normal distribution. Next, for an event $A$, let $\mathbb{I}_{A}$ be the indicator of $A$, that is,

$$
\mathbb{I}_{A}(\mathbf{X})=1 \text { if } \mathbf{X} \in A \text {, and } \mathbb{I}_{A}(\mathbf{X})=0 \text { if } \mathbf{X} \notin A
$$

Then, we define the statistic $\left[T_{n}(d)\right]_{P T E}=\left[T_{n}(d)\right]_{P T E}(\mathbf{X})$ as

$$
\left[T_{n}(d)\right]_{P T E}=T_{n}(\hat{d}) \mathbb{I}_{A_{n}^{\prime}}+T_{n}(0) \mathbb{I}_{A_{n}}
$$

$\mathrm{R}$ function for computing $\left[T_{n}(d)\right]_{P T E}$ is given in the Appendix.

The next result establishes the asymptotic distribution of $\left[T_{n}(d)\right]_{P T E}$.

Theorem 4.2. Let the sequence $\left\{X_{t}\right\}$ satisfy the assumptions of Theorem 4.1 related to $V / S$ statistic and the conditions on a Whittle estimator in Section 3.4. Then for all $d \in(-1 / 2,1 / 2)$

$$
\left[T_{n}(d)\right]_{P T E} \stackrel{D}{\longrightarrow} Z_{d}
$$

where

$$
Z_{d}=\int_{0}^{1}\left(W_{1 / 2+d}^{0}(t)-\int_{0}^{1} W_{1 / 2+d}^{0}(u) d u\right)^{2} d t
$$


with $W_{1 / 2+d}^{0}(t)$ being a fractional Brownian bridge.

Proof. Due to the fact that $T_{n}(\hat{d}) \stackrel{D}{\longrightarrow} Z_{d}$ and $T_{n}(0) \stackrel{D}{\longrightarrow} Z_{0}$, we only need to show that the following two relations hold:

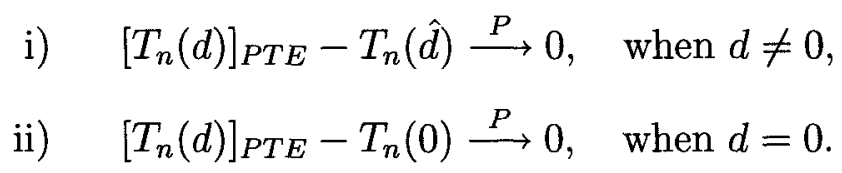

Proof of i). By the definition of $\left[T_{n}(d)\right]_{P T E}$,

$$
\left[T_{n}(d)\right]_{P T E}-T_{n}(\hat{d})=T_{n}(\hat{d}) \mathbb{I}_{A_{n}^{\prime}}+T_{n}(0) \mathbb{I}_{A_{n}}-T_{n}(\hat{d})=\left[T_{n}(0)-T_{n}(\hat{d})\right] \mathbb{I}_{A_{n}},
$$

and therefore

$$
\begin{aligned}
\mathbf{P}\left(\left|\left[T_{n}(d)_{P T E}\right]-T_{n}(\hat{d})\right|>\epsilon\right) & =\mathbf{P}\left[\left|T_{n}(0)-T_{n}(\hat{d})\right| \mathbb{I}_{A_{n}}>\epsilon\right] \leq \mathbf{P}\left(A_{n}\right) \\
& =\mathbf{P}\left(2 \sqrt{m}|\hat{d}| \leq \Phi^{-1}(1-\alpha / 2)\right) \rightarrow 0 \text { as } n \rightarrow \infty
\end{aligned}
$$

since $2 \sqrt{m}(\hat{d}-d) \stackrel{D}{\longrightarrow} N(0,1)$, which implies that $2 \sqrt{m} \hat{d} \rightarrow \infty$ in probability.

Proof of ii). We have

$$
\begin{aligned}
\left.\mathbf{P}\left(\mid T_{n}(d)_{P T E}\right]-T_{n}(\hat{d}) \mid>\epsilon\right) & =\mathbf{P}\left[\left|T_{n}(0)-T_{n}(\hat{d})\right| \mathbb{I}_{A_{n}}>\epsilon\right] \\
& \leq \mathbf{P}\left[\left|T_{n}(0)-T_{n}(\hat{d})\right|>\epsilon\right] \rightarrow 0
\end{aligned}
$$

since $T_{n}(d)-T_{n}(\hat{d}) \rightarrow 0$ in probability when $\hat{d}-d=o_{P}(1 / \log (n))$, (see Giraitis et al. (2006)). This is the case here, since $\sqrt{m} \geq \log n$.

Remark: By using the PTE method, it is possible to decrease the Type I error probability, i.e., the probability of rejecting the null hypothesis when it is true. 


\section{Comparison of Tests and Empirical Studies}

\subsection{Method of Comparison}

In this section, we shall compare the tests described in the previous sections to determine which test statistic is better for testing the short memory null hypothesis versus a long memory alternative.

In modern statistic, along with the Neyman-Pearson idea of defining a "best test", there is another approach based on the total error probability. To choose the best test among the test procedures discussed in this thesis, we use the criterion based on the total error probability. In connection with this criterion, a commonly used approach is the minimax one. Roughly speaking, a test considered to be the best one if its maximal total error probability is the smallest possible among all tests. If the null hypothesis is simple, then the maximal total error probability is just the sum of the Type I error probability and the maximal (over all alternative values of the parameter) Type II error probability. We suggest to adjust this theoretical approach to our empirical study as follows. The quality of testing is measured by looking at the sum of the empirical size and the maximum of one minus the empirical

power. The test for which this sum is the smallest will be the best (among the tests under consideration). For details on the minimax approach based on the total error probability, see Chapter 2 in Ingster and Suslina (2002).

\subsection{Short Memory versus Long Memory}

The results of our simulation study are collected in Tables 6-13, where Table 6 provides the comparison study results.

As the null hypothesis for computing empirical size of a test we take an $\operatorname{AR}(1)$ 
process. To simulate the realization of this process, we use $\mathrm{R}$ function "arima.sim" from the statistical library \{stats\}. As an alternative, for computing empirical power of a test, we take a $\operatorname{FARIMA}(0, d, 0)$ process. To simulate its realization, we use $\mathrm{R}$ function "fracdiff.sim" from the library \{fracdiff\}. For the study of modified R/S, KPSS, and V/S test statistics, we use 10,000 replications of each test and work with samples of size 500 . We take a window parameter to be $m=n^{0.7}$. In the empirical size study, we simulate a sample from the null hypothesis of short range stationarity model and compute the probability of rejecting the null hypothesis. In the empirical power study, we simulate a sample from the long memory alternative, then run tests under the null hypothesis of short memory and compute the probability of rejecting $H_{0}$.

When using our PTE test statistic, we need to estimate the long memory parameter $d$. Because of much time required to get an estimate of $d$, we base our simulation on 200 replications (instead of 10,000 replications in the case of modified R/S, KPSS, and $\mathrm{V} / \mathrm{S}$ statistics) and keep the same sample size $n=500$. We also limit the values of a window parameter to $q=\{1,3,5\}$. Obviously, with a smaller number of replications the accuracy drops significantly.

Adjusting the minimax approach to our empirical study, we selected (with no particular preference) two sets of values for $\alpha$ and $q$ from Tables 7-12 below that give the values of empirical size and power. Namely, $\alpha=5 \%, q=3$ and $\alpha=5 \%, q=5$. The values of maximal total error probabilities of the modified R/S, KPSS, and V/S tests are given in Table 6. Note that, unlike the minimax total error probability, the maximal total error probability of a particular test does not need to be less than or equal to 1 . 
Table 6: Total error probabilities of the modified R/S, KPSS, and V/S tests, in percentage.

\begin{tabular}{cccc}
\hline$q=3, \alpha=5 \%(q=5, \alpha=5 \%)$ & Modified R/S & KPSS & V/S \\
Type I Error Probability & $53.64(30.89)$ & $35.47(24.66)$ & $51.37(35.01)$ \\
Type II Error Probability & $48.61(58.44)$ & $59.43(65.09)$ & $47.48(54.69)$ \\
\hline Total Error Probability & $102.25(89.33)$ & $94.90(89.75)$ & $98.85(89.70)$
\end{tabular}

According to Table 6 , for the selected sets of values of $q$ and $\alpha$, the KPSS test has the smallest maximal total error probability and therefore is considered to be the best one. A possible interpretation of this is the following. The KPSS test already had a very good empirical power. The V/S test has even better power, but it comes at the expense of a higher empirical size. Therefore, according to the criterion that we use, with a focus on both Type I and Type II error probabilities, the V/S test it not the best.

Remark: We do not include the PTE test into this comparison study because, unlike the other tests, its tables are based on $\hat{d}$, an estimate of $d$. In addition, we had to use a much smaller number of replications (200 instead of 10,000). Nevertheless, its empirical size table (Table 13) shows that the test based on $\left[T_{n}(d)\right]_{P T E}$ has a very low Type I error probability. 
Table 7: Empirical size of the modified $R / S$ test, in percentage.

\begin{tabular}{cccccccc}
\hline & \multicolumn{2}{c}{$\phi=0.2$} & \multicolumn{2}{c}{$\phi=0.5$} & \multicolumn{2}{c}{$\phi=0.8$} \\
\hline $\mathrm{N}$ & $\mathrm{q}$ & $10 \%$ & $5 \%$ & $10 \%$ & $5 \%$ & $10 \%$ & $5 \%$ \\
\hline 500 & 0 & 27.90 & 17.92 & 77.58 & 66.97 & 99.82 & 99.37 \\
& 1 & 16.45 & 8.71 & 46.36 & 33.94 & 93.69 & 88.18 \\
& 3 & 10.24 & 4.80 & 21.93 & 13.14 & 65.54 & 53.64 \\
& 5 & 8.89 & 4.20 & 14.38 & 7.64 & 43.53 & 30.89
\end{tabular}

Table 8: Empirical size of the KPSS test, in percentage.

$\begin{array}{rrrrrrrr}500 & 0 & 21.55 & 13.53 & 51.62 & 37.04 & 93.29 & 85.61 \\ 1 & 16.32 & 8.48 & 31.94 & 20.93 & 74.05 & 62.56 \\ 3 & 12.52 & 6.55 & 19.90 & 12.01 & 49.63 & 35.47 \\ 5 & 11.22 & 6.26 & 15.85 & 9.19 & 36.25 & 24.66\end{array}$

Table 9: Empirical size of the $V / S$ test, in percentage.

$\begin{array}{rrrrrrrr}500 & 0 & 26.87 & 15.11 & 68.80 & 55.24 & 99.42 & 97.74 \\ 1 & 17.39 & 9.24 & 43.02 & 30.20 & 90.84 & 82.80 \\ 3 & 13.14 & 7.01 & 24.56 & 14.25 & 65.64 & 51.37 \\ 5 & 11.74 & 5.66 & 18.37 & 9.65 & 48.48 & 35.01\end{array}$


Table 10: Empirical power of the modified $R / S$ test, in percentage.

\begin{tabular}{cccccccc}
\hline & \multicolumn{4}{c}{$d=0.2$} & \multicolumn{2}{c}{$d=0.3$} & \multicolumn{2}{c}{$d=0.4$} \\
\hline $\mathrm{N}$ & $\mathrm{q}$ & $10 \%$ & $5 \%$ & $10 \%$ & $5 \%$ & $10 \%$ & $5 \%$ \\
\hline 500 & 0 & 86.45 & 79.56 & 98.20 & 96.16 & 99.82 & 99.56 \\
& 1 & 76.26 & 67.44 & 93.93 & 90.75 & 98.85 & 97.65 \\
& 3 & 62.46 & 51.39 & 83.76 & 76.15 & 94.61 & 90.85 \\
& 5 & 53.78 & 41.56 & 74.24 & 64.86 & 88.31 & 81.41
\end{tabular}

Table 11: Empirical power of the KPSS test, in percentage.

$\begin{array}{rrrrrrrr}500 & 0 & 71.32 & 59.07 & 91.09 & 83.85 & 97.68 & 95.87 \\ 1 & 62.71 & 50.22 & 84.59 & 74.72 & 94.51 & 89.84 \\ & 3 & 52.64 & 40.57 & 73.77 & 63.61 & 87.36 & 79.59 \\ & 5 & 46.85 & 34.91 & 66.64 & 54.52 & 80.77 & 70.80\end{array}$

Table 12: Empirical power of the V/S test, in percentage.

$\begin{array}{rlllllll}500 & 0 & 82.71 & 74.19 & 96.85 & 93.89 & 99.65 & 99.13 \\ 1 & 75.16 & 64.45 & 92.58 & 87.95 & 98.60 & 96.65 \\ 3 & 64.03 & 52.52 & 83.97 & 76.42 & 94.70 & 89.83 \\ 5 & 57.05 & 45.31 & 77.33 & 66.85 & 89.62 & 82.65\end{array}$


Table 13: Empirical size of the 5\% test based on the 10\% and 5\% PTE statistic, in percentage

\begin{tabular}{ccccc}
\hline & \multicolumn{2}{c}{$\phi=0.2$} & \multicolumn{2}{c}{$\phi=0.5$} \\
\hline $\mathrm{q}$ & $10 \%$ & $5 \%$ & $10 \%$ & $5 \%$ \\
\hline 1 & 13.5 & 9.05 & 6.50 & 4.00 \\
3 & 11.00 & 4.00 & 3.5 & 2.00 \\
5 & 9.00 & 2.50 & 3.00 & 1.00 \\
\hline
\end{tabular}




\section{Conclusion}

In this thesis, we aimed at carrying out thorough investigation and improvement of some statistical tests used to differentiate between the slow decay of the covariances that might be attributed to the lack of stationarity and the one due to long or short range dependence. We were especially interested in detecting a long range dependence pattern in a time series data. Such a detection is pursued either via direct testing, or via estimation of the so-called long memory parameter. Various approaches to the long memory detection problem and various statistics were considered, including the modified R/S, KPSS, and V/S statistics. Using the preliminary test estimation (PTE) approach, we have also introduced a new test statistic and established its asymptotic distribution under the short and long range dependence assumptions. We have compared all these test statistics based on their empirical sizes and powers, and also by looking at their empirical maximal total error probabilities. It turned out that the test based on KPSS statistic tends to be the best one in most of the cases, although the difference between the KPSS and V/S tests is not that big. A newly proposed PTE test seems to produce small Type I error probabilities, which was the aim of introducing it (to reduce high empirical size of the V/S test). However, in my opinion, we shall have to refine on this PTE test statistic because it is based on a Whittle estimator of $d$, even under $H_{0}$ of short range dependence assumption. This estimator is not very accurate under $H_{0}$, and, in general, its convergence rate $\sqrt{m}$ is much slower than the usual $\sqrt{n}$. This drawback of a Whittle estimator often makes the PTE test tending to choose an unrestricted estimator $T_{n}(\hat{d})$, even in case of sampling under the null hypothesis of short memory. 


\section{Appendix}

A1. This function estimates the long memory parameter $d$ based on the R/S statistic. The function has one parameter $x$, the name of the sample.

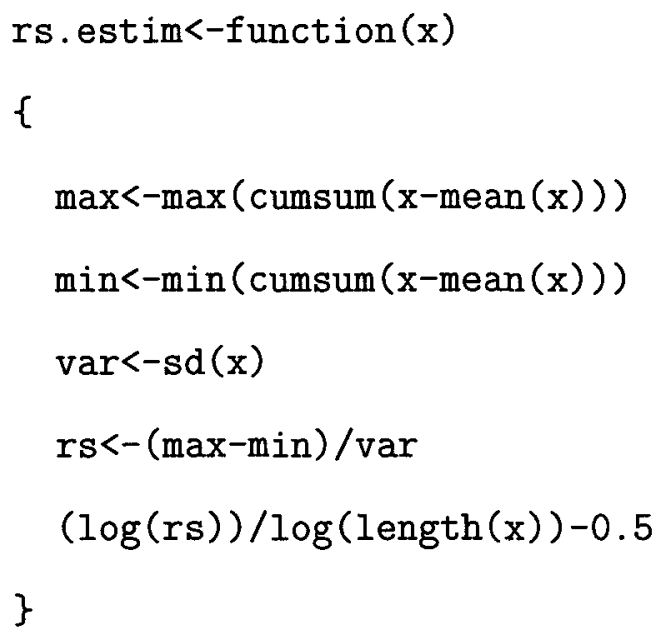

A2. This function estimates the long memory parameter $d$ based on the GPH statistic. The function has one parameter $x$, the name of the sample.

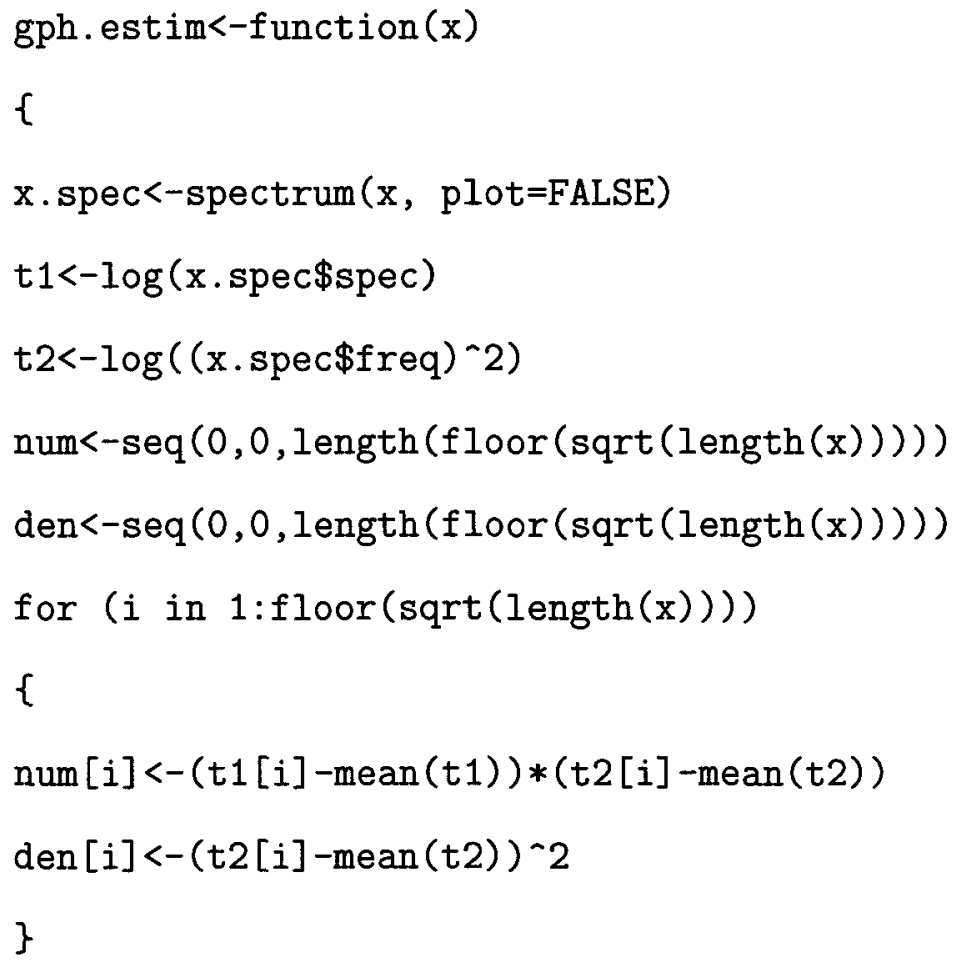


$-\operatorname{sum}($ num) $/$ sum (den)

\}

A3. This function estimates the long memory parameter $d$ based on a Whittle statistic. The function has one parameter $x$, the name of the sample.

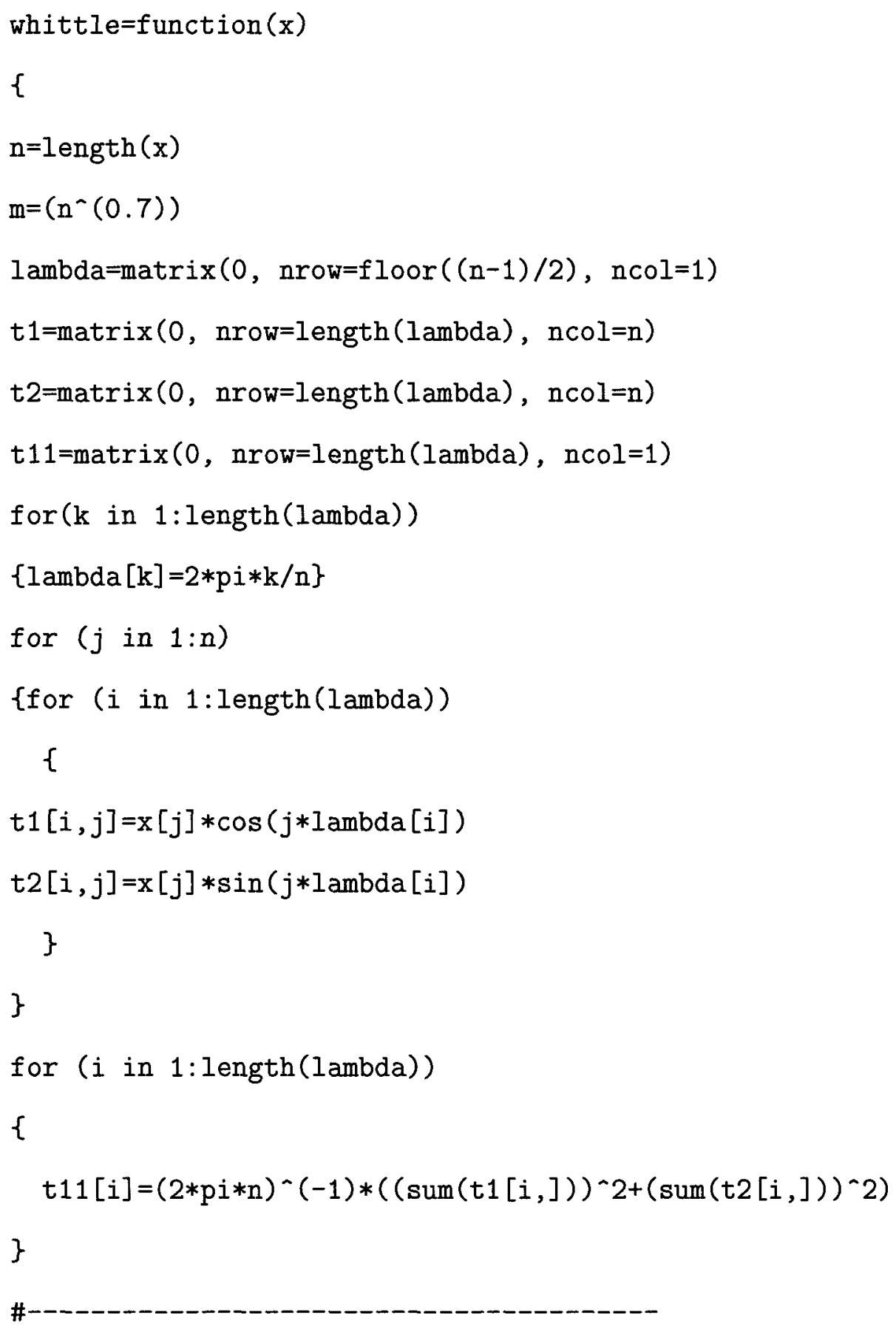




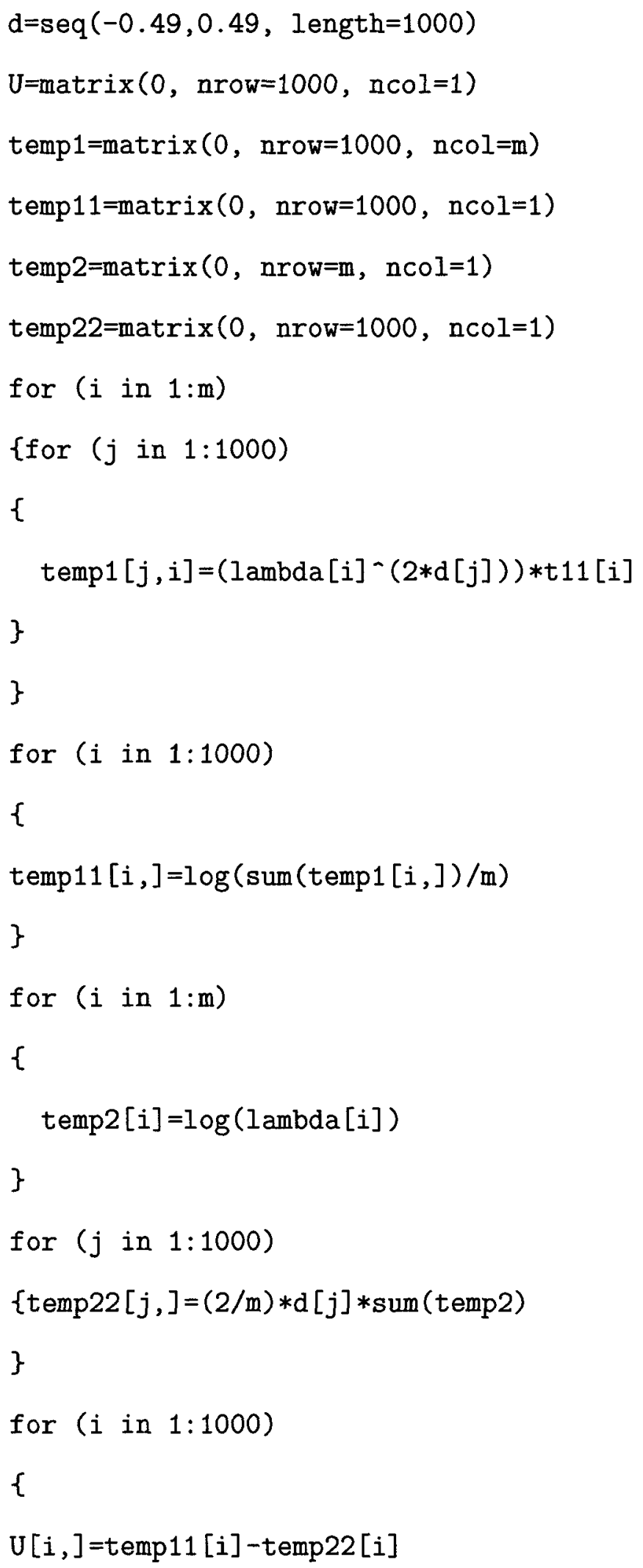


$\mathrm{d}[$ which $\cdot \min (\mathrm{U})]$

\}

A4. This function computes the value of modified R/S statistic under the null hypothesis of short range dependence $(d=0)$. The function has two parameters: $x$ is the name of the sample and $q$ is a window parameter.

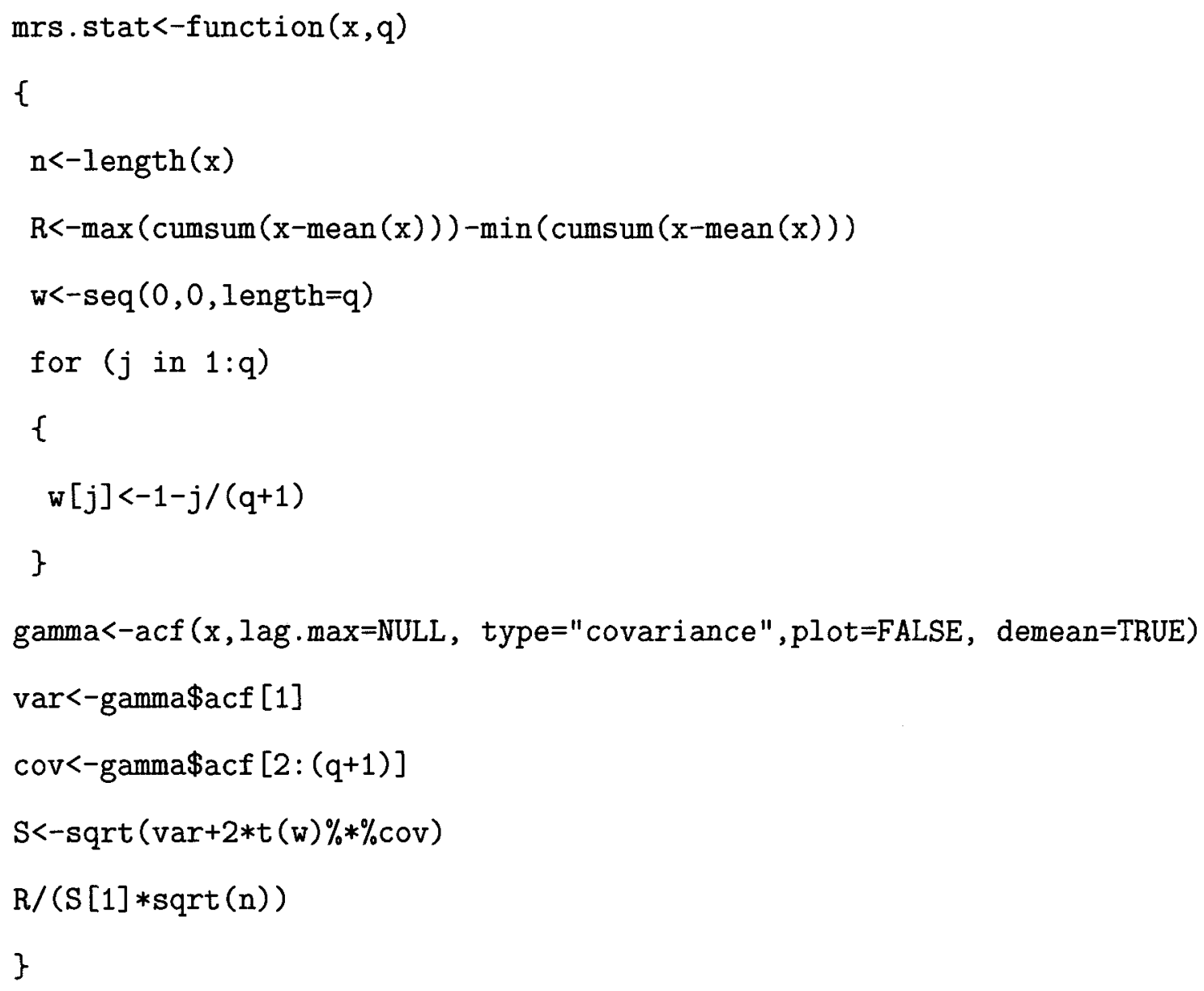

A5. This function computes the value of KPSS statistic under the null hypothesis of short range dependence $(d=0)$. The function has two parameters: $x$ is the name of the sample, and $q$ is a window parameter.

kpss. $\operatorname{stat}=f$ unction $(x, q)$ 


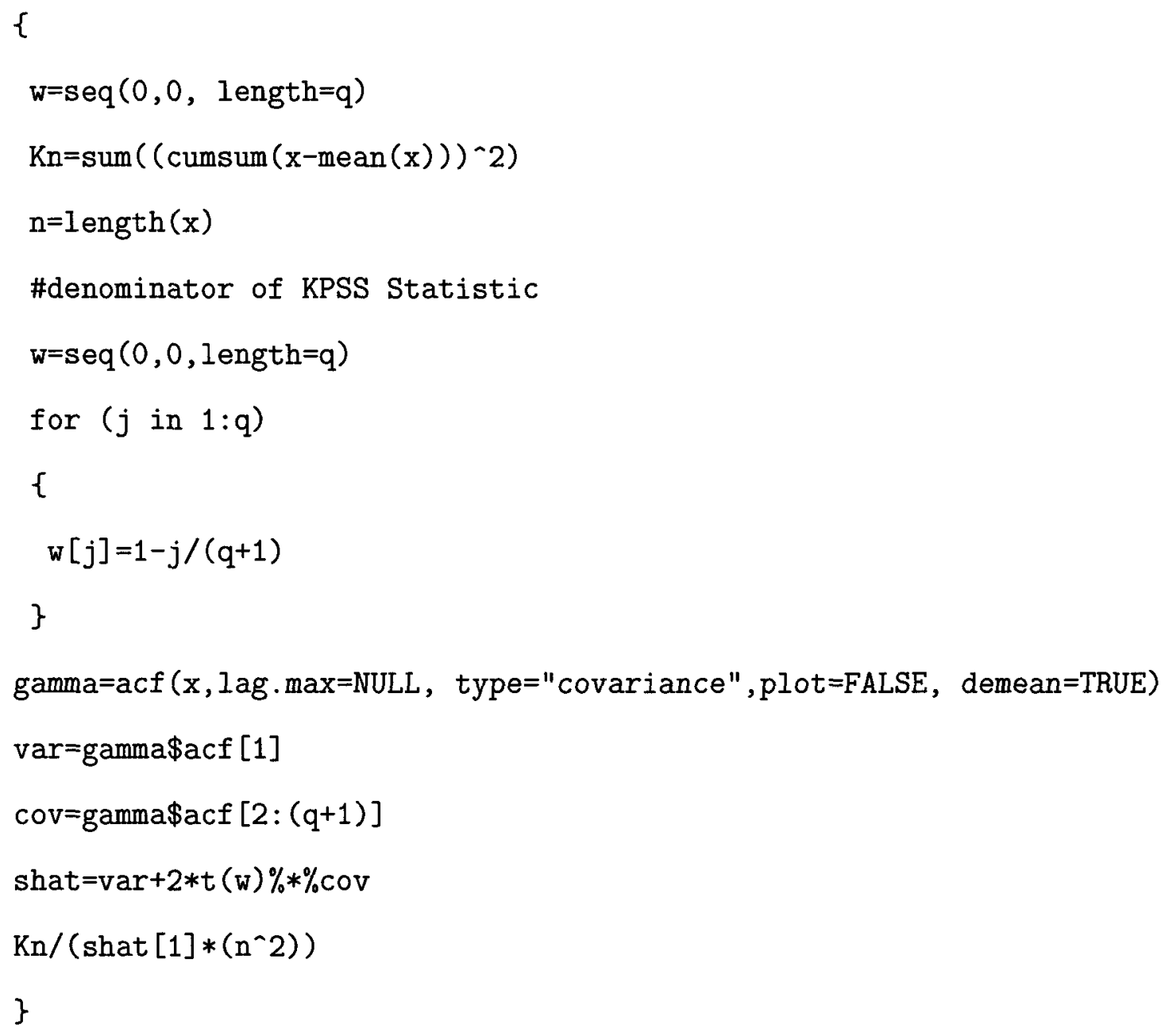

A6. This function computes the value of $\mathrm{V} / \mathrm{S}$ statistic under the null hypothesis of short range dependence $(d=0)$. The function has three parameters: $x$ is the name of the sample, dhat (or $\hat{d}$ ) is an estimate of the long memory parameter $d$. It is assumed that a Whittle estimator will be used. So, before running the function vs.stat, it is necessary to load the function whittle and put whittle $(x)$ instead of dhat in the function vs.stat. The third parameter $q$ is a window parameter.

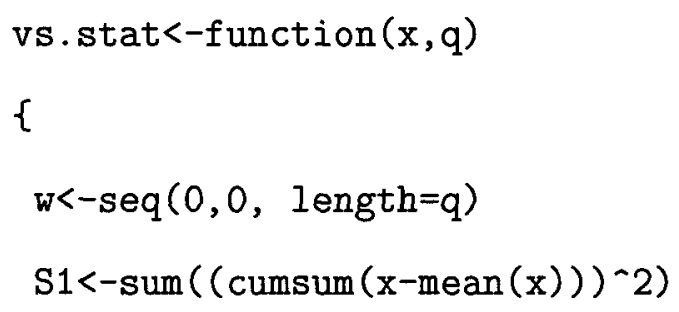




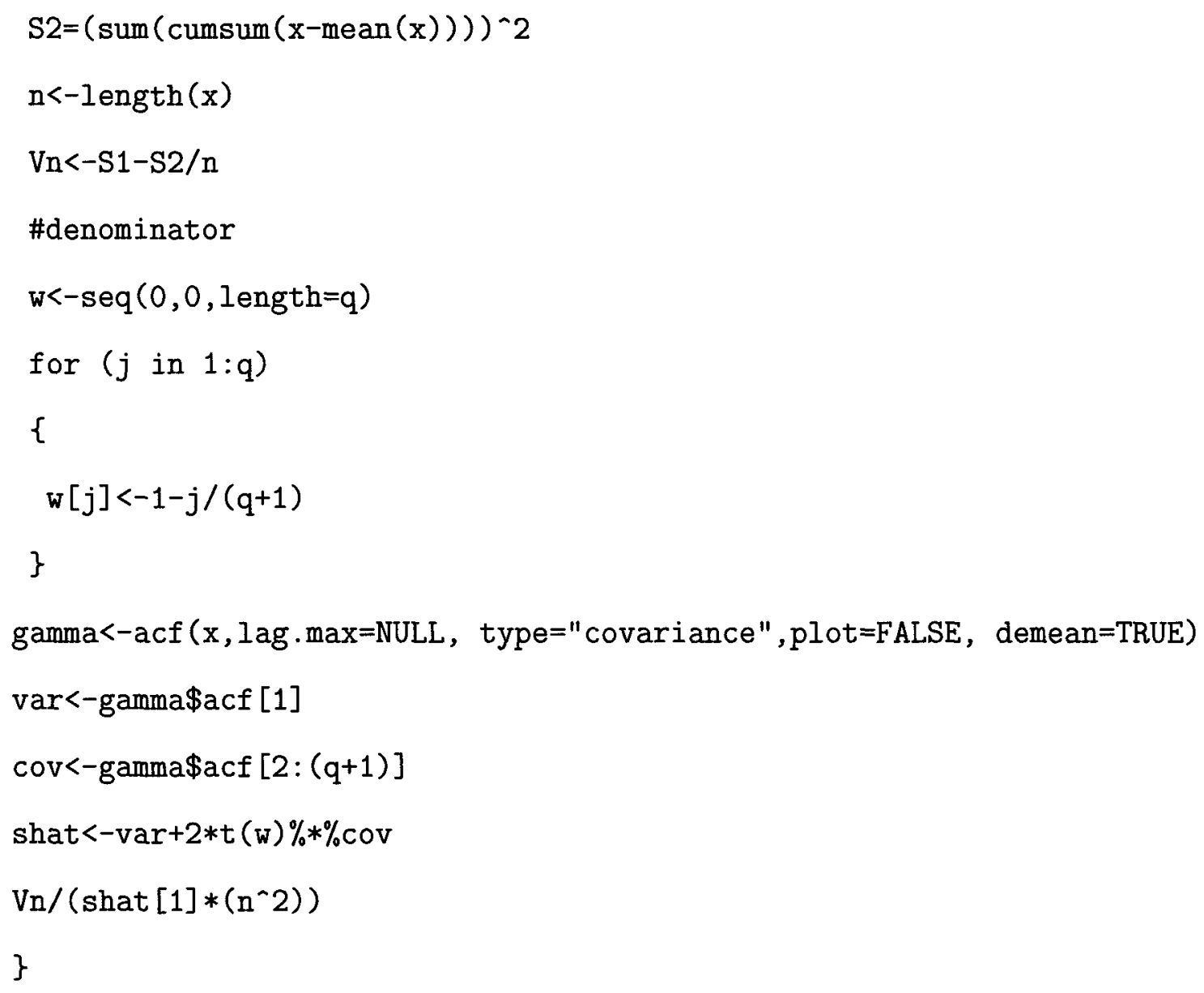

A7. This function computes the value of V/S statistic under the alternative hypothesis of long memory, $0<d<1 / 2$. The function has three parameters: $x$ is the name of the sample, $\hat{d}$ is an estimate of the long memory parameter $d$, and $q$ is a window parameter.

lvs.stat<-function( $x$, dhat, $q)$

\{

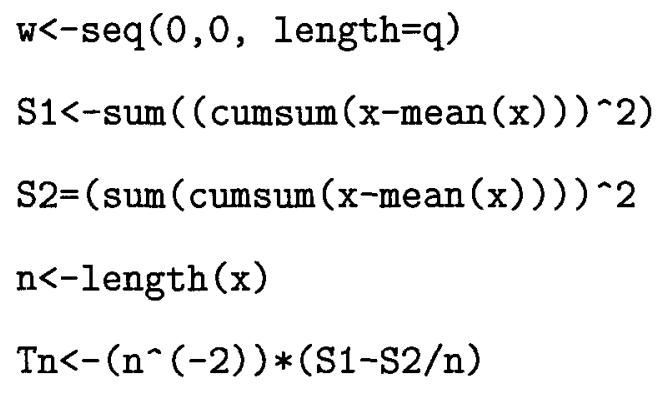




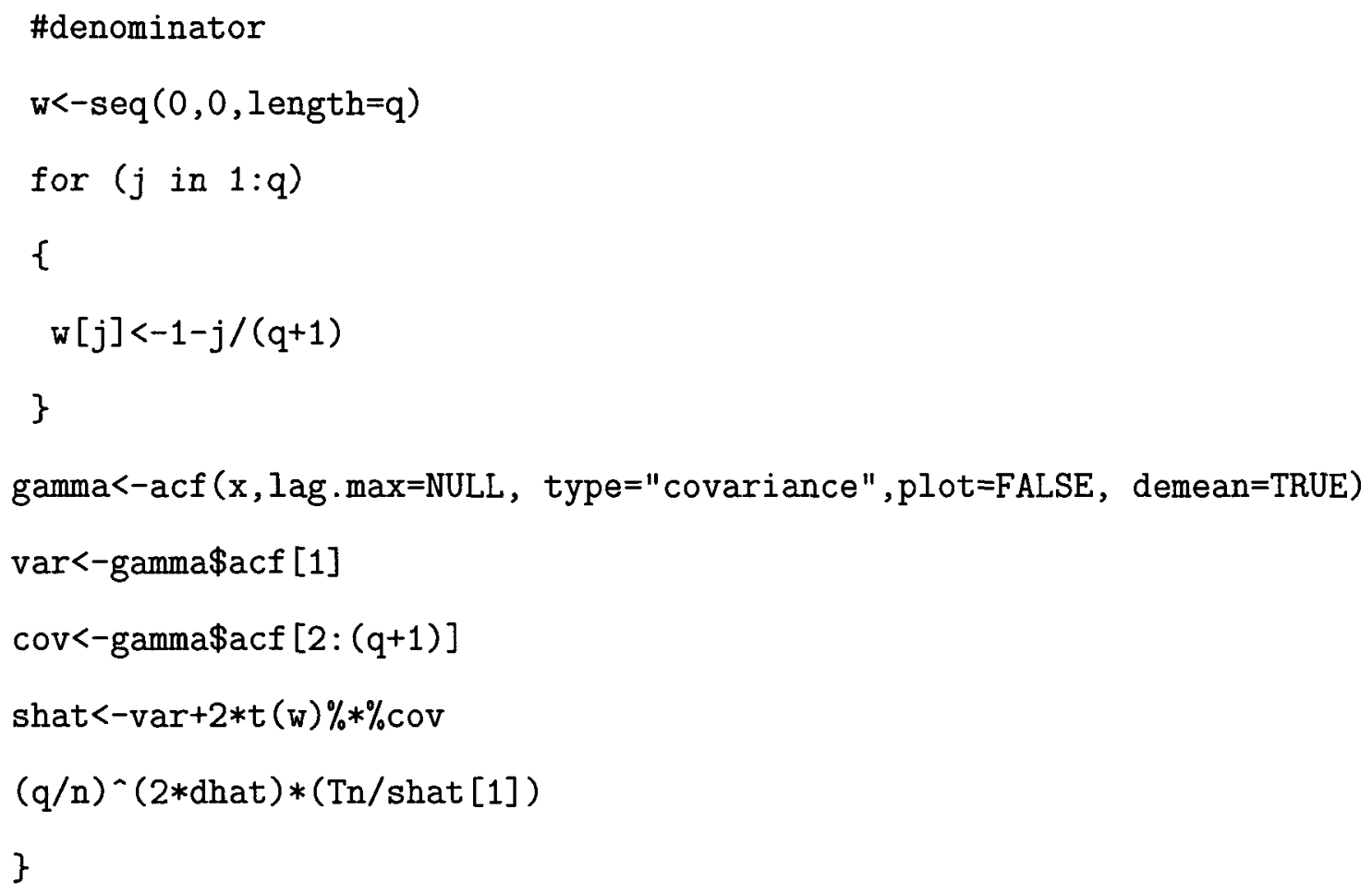

A8. This function computes the value of PTE statistic. The function has three parameters: $x$ is the name of the sample, $q$ is a window parameter, and $\alpha$ is the significance level of the preliminary test for testing $H_{0}: d=0$ vs. $H_{a}: d \neq 0$. The PTE statistic is based on V/S statistic. Therefore, before running pte.stat, one has to load the functions whittle, vs.stat, and lvs.stat.

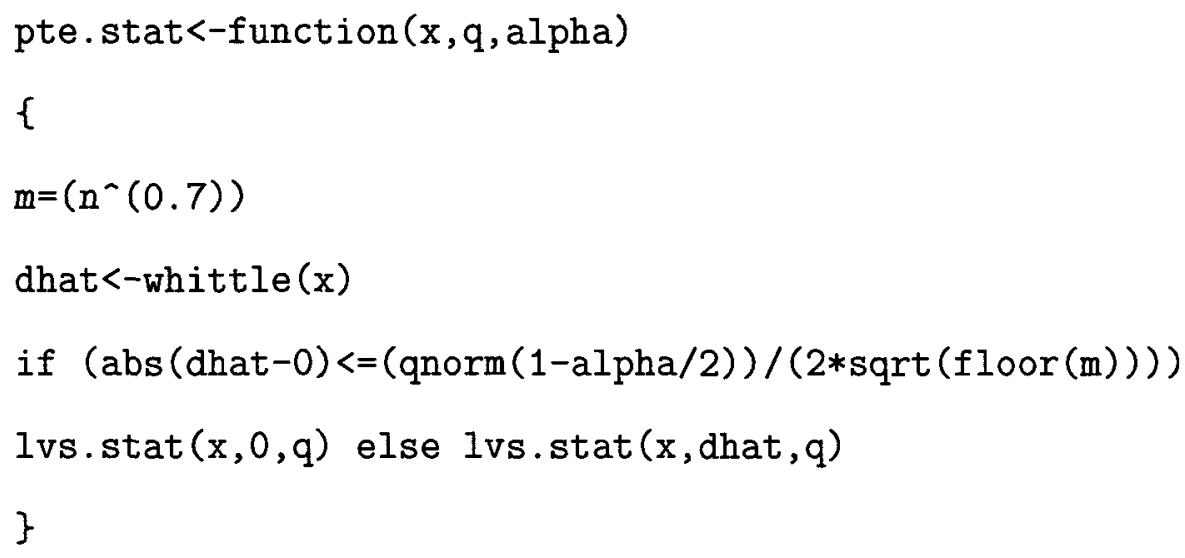


A9. This code is used for computing empirical size of the modified R/S test for testing the short memory null hypothesis versus the long memory alternative. Before running the code, one has to load the functions in A3-A8.

$f i=g$ ive the value of autocovariance coefficient: $0.2,0.5,0.8$.

$n=g i v e$ the sample size. In this thesis used sample size is 500 . $q=g i v e$ the value of a window parameter, in this thesis $q=0,1,3,5$. $r=g i v e$ the number of replications. We use 10,000 replications. qmrs=give the quantiles of modified R/S: $10 \%-1.620,5 \%-1.747$.

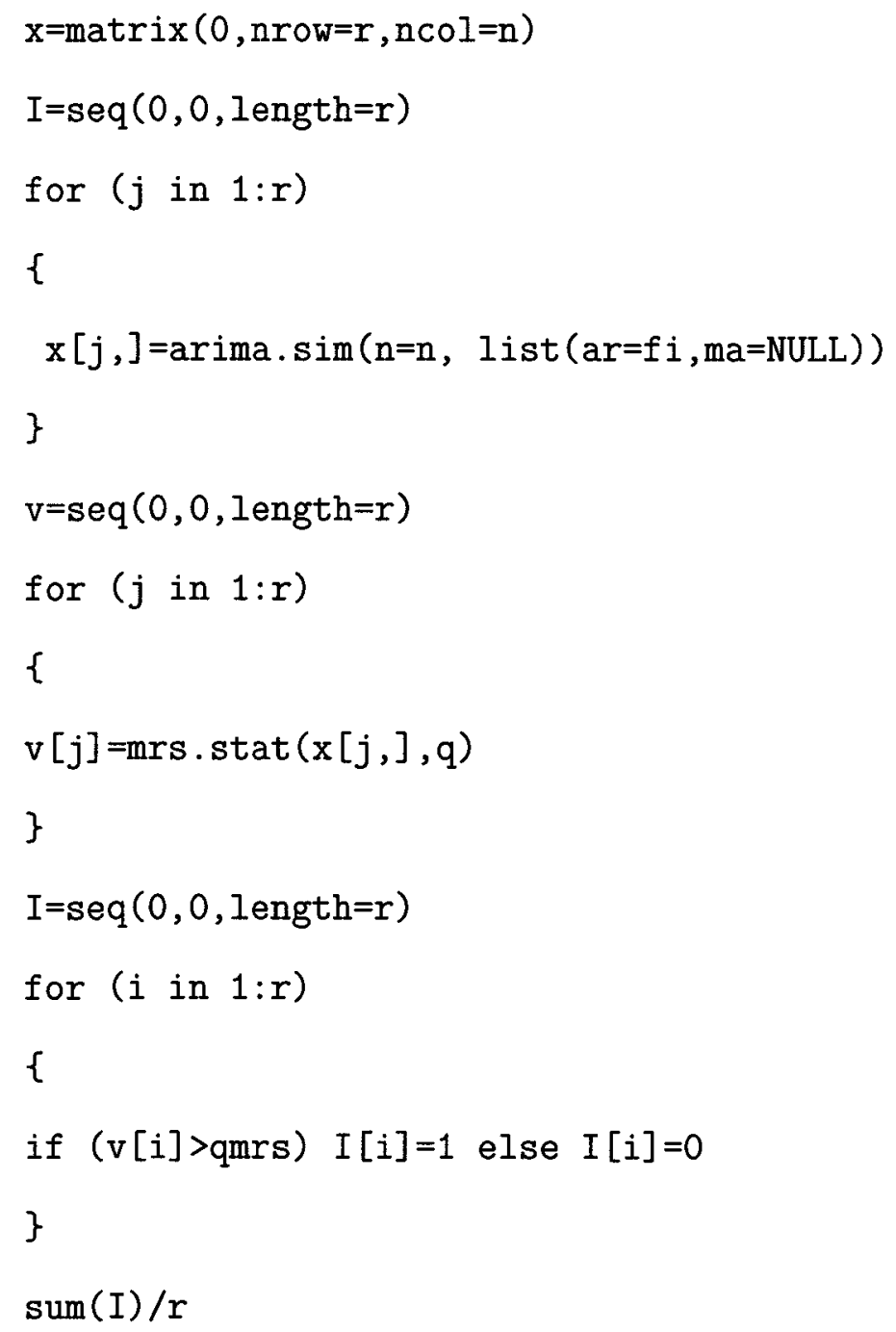


A10. This code is used for computing empirical power of the modified R/S test for testing the short memory null hypothesis versus the long memory alternative. Before running the code, one has to load the functions in A3-A8.

$d=$ give the value of long memory parameter: $d=0.2,0.3,0.4$.

$\mathrm{n}=$ give the sample size. In this thesis used sample size is 500 .

$\mathrm{q}=$ give the value of a window parameter: $\mathrm{q}=0,1,3,5$ are used.

$r=g i v e$ the number of replications. We use 10,000 replications.

qmrs=give the quantiles of modified R/S: $10 \%-1.620,5 \%-1.747$.

library (fracdiff)

$\mathrm{x}=\operatorname{matrix}(0$, nrow $=\mathrm{r}, \mathrm{ncol}=\mathrm{n})$

$I=\operatorname{seq}(0,0$, length $=r)$

for $(j$ in $1: r)$

\{

$x[j]=$, fracdiff. $\operatorname{sim}(n=n$, ar $=$ NULL, $m a=N U L L, d=d) \$$ series

\}

$\mathrm{v}=\operatorname{seq}(0,0$, length $=r)$

for $(j$ in $1: r)$

\{

$v[j]=\operatorname{mrs} . \operatorname{stat}(x[j], q$,

\}

$I=\operatorname{seq}(0,0$, length $=r)$

for ( $i$ in $1: r$ )

\{

if $(v[i]>q m r s) ~ I[i]=1$ else $I[i]=0$

\}

$\operatorname{sum}(I) / r$ 
A11. This code is used for computating empirical size of the KPSS test for testing the short memory null hypothesis versus the long memory alternative. Before running the code, one has to load the functions in A3-A8.

$f i=g i v e$ the value of autocovariance coefficient: $0.2,0.5,0.8$. $\mathrm{n}=$ give the sample size. In this thesis used sample size is 500 . $q=g i v e$ the value of a window parameter, in this thesis $q=0,1,3,5$. $r=$ give the number of replications. We use 10,000 replications. qkpss=give the quantiles of KPSS. $10 \%-0.34730,5 \%-0.46136$. $\mathrm{x}=\operatorname{matrix}(0$, nrow $=\mathrm{r}, \mathrm{ncol}=\mathrm{n})$

$I=\operatorname{seq}(0,0$, length $=r)$

for $(j$ in $1: r)$

\{

$x[j]=,\operatorname{arima} . \operatorname{sim}(n=n$, list $(\operatorname{ar}=f i, m a=N U L L))$

\}

$\mathrm{v}=\operatorname{seq}(0,0$, length $=r)$

for ( $j$ in $1: r$ )

\{

$v[j]=\operatorname{kpss} . \operatorname{stat}(x[j], q$,

\}

$I=\operatorname{seq}(0,0$, length $=r)$

for ( $i$ in $1: r$ )

\{

if $(v[i]>q k p s s) \quad I[i]=1$ else $I[i]=0$

\}

$\operatorname{sum}(I) / r$ 
A12. This code is used for computing empirical power of the KPSS test for testing the short memory null hypothesis versus the long memory alternative. Before running the code, one has to load the functions in A3-A8.

$d=$ give the value of long memory parameter: $d=0.2,0.3,0.4$.

$\mathrm{n}=$ give the sample size. In this thesis used sample size is 500 .

$\mathrm{q}=$ give the value of a window parameter: $\mathrm{q}=0,1,3,5$ are used.

$r=g i v e$ the number of replications. We use 10,000 replications.

qkpss=give the quantiles of KPSS. $10 \%-0.34730,5 \%-0.46136$.

library (fracdiff)

$\mathrm{x}=$ matrix $(0$, nrow $=\mathrm{r}, \mathrm{ncol}=\mathrm{n})$

$I=\operatorname{seq}(0,0$, length $=r)$

for $(j$ in $1: r$ )

\{

$x[j]=$, fracdiff. $\operatorname{sim}(n=n$, ar=NULL, ma=NULL, $d=d) \$$ series

\}

$v=\operatorname{seq}(0,0$, length $=r)$

for $(j$ in $1: r$ )

\{

$v[j]=\operatorname{kpss} . \operatorname{stat}(x[j], q$,

\}

$I=\operatorname{seq}(0,0$, length $=r)$

for ( $i$ in $1: r$ )

\{

if ( $[i]>q k p s s) ~ I[i]=1$ else $I[i]=0$

\}

$\operatorname{sum}(I) / r$ 
A13. This code is used for computing empirical size of the $\mathrm{V} / \mathrm{S}$ test for testing the short memory null hypothesis versus the long memory alternative. Before running the code, one has to load the functions in A3-A8.

$f i=g i v e$ the value of autocovariance coefficient: $0.2,0.5,0.8$. $\mathrm{n}=\mathrm{give}$ the sample size. In this thesis used sample size is 500 . $q=g i v e$ the value of a window parameter: $q=0,1,3,5$. $r=$ give the number of replications. We use 10,000 replications. qvs=give the quantiles of $\mathrm{V} / \mathrm{S}: 10 \%-0.153,5 \%-0.190$. 
A14. This code is used for computing empirical power of the V/S test for testing the short memory null hypothesis versus the long memory alternative. Before running the code, one has to load the functions in A3-A8.

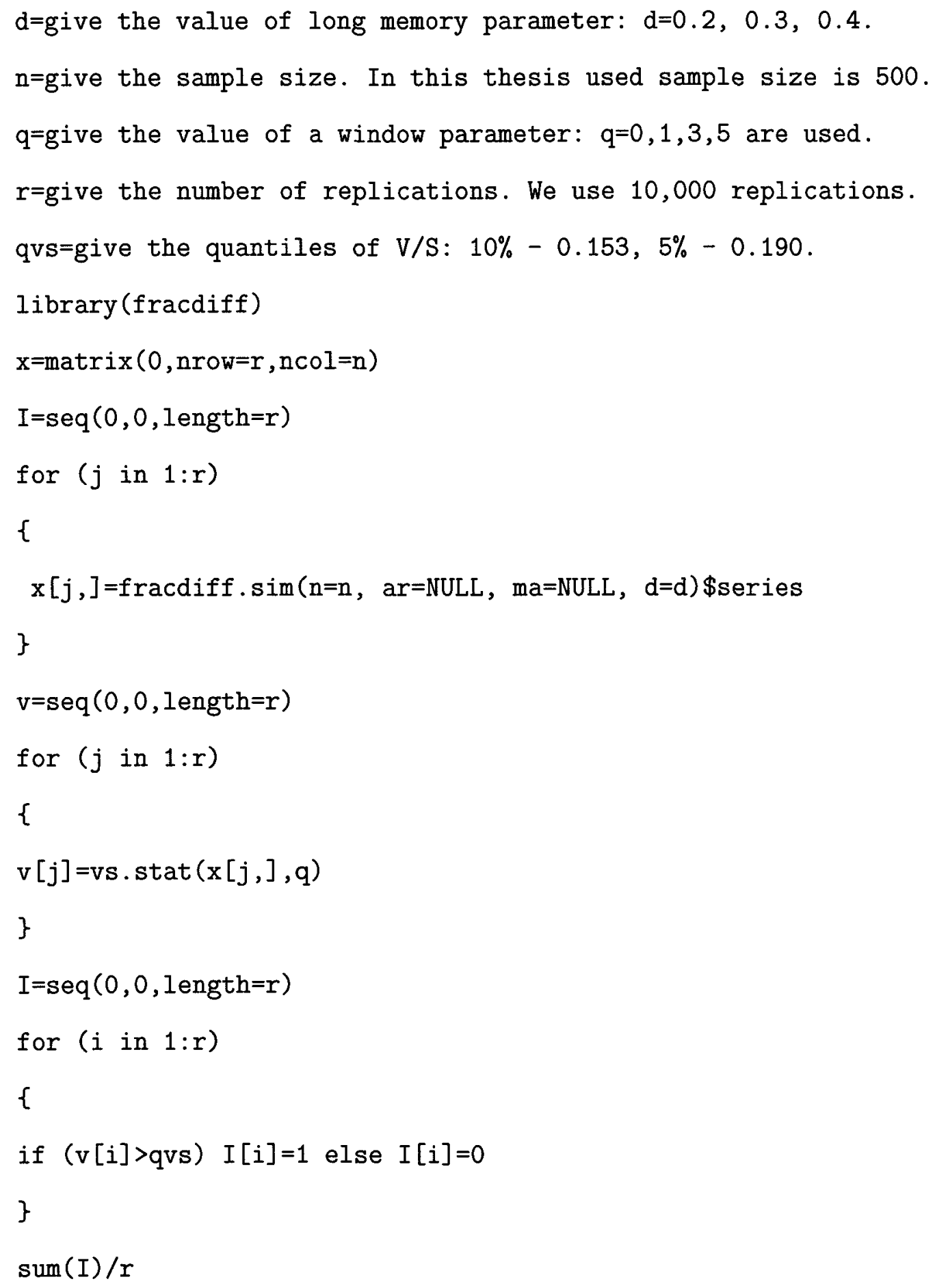




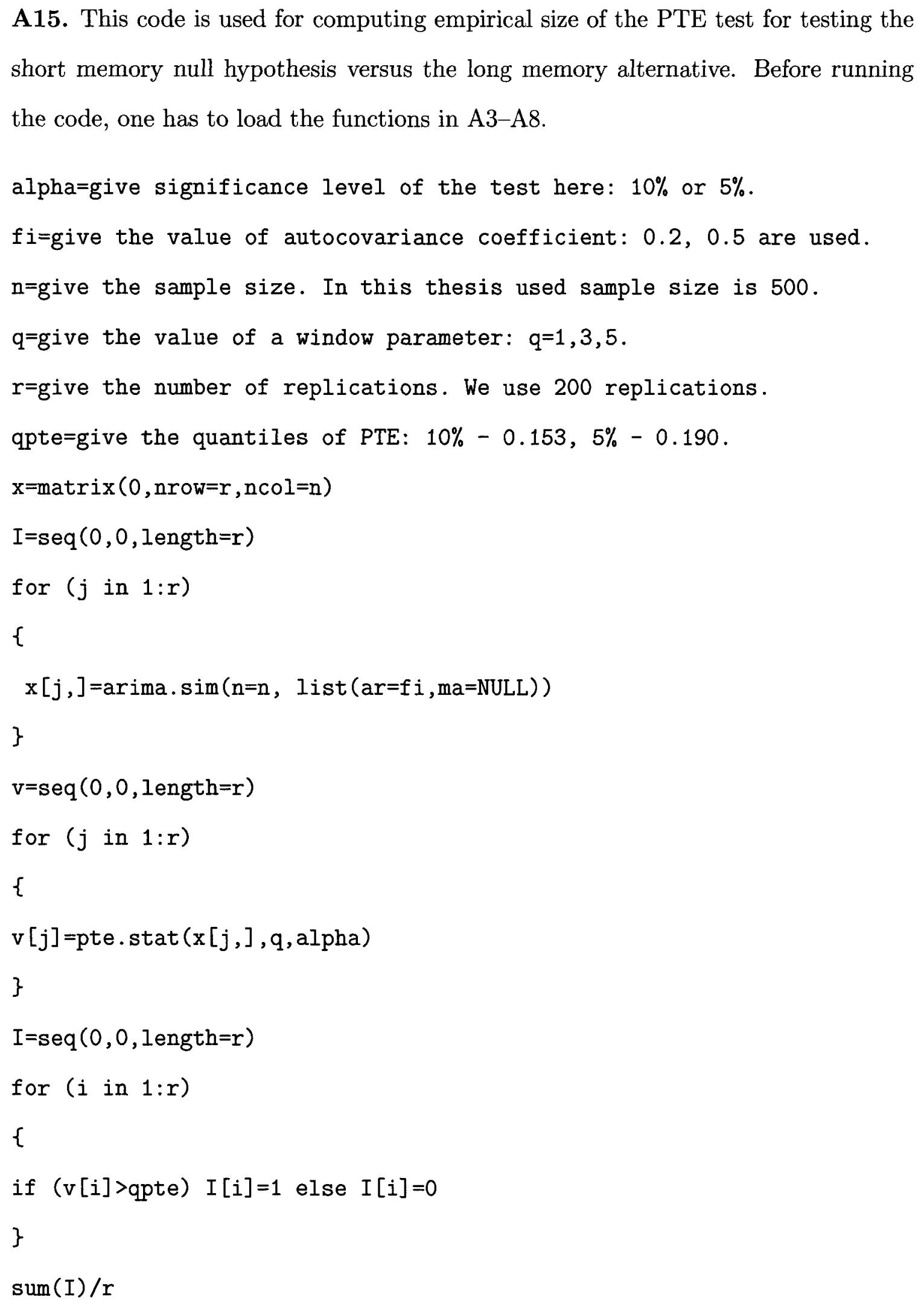




\section{References}

[1] D.W.K. Andrews (1991). Heteroskedasticity and autocorrelation consistent covariance matrix estimation. Econometrica, 59, 817-858.

[2] J. Beran (1994). Statistics for Long-Memory Processes. Chapman \& Hall/CRC.

[3] P.J. Brockwell and R.A. Davis (1991). Time Series: theory and methods. Springer, New York.

[4] I.N. Bronshtein, K.A. Semendyayev, G. Musiol, and H. Muehlig (2000). Handbook of Mathematics, 4th Ed., Springer, New York.

[5] H. Cremers and D. Kadelka (1986). On weak convergence of integral functionals of stochastic processes with applications to processes taking paths in $l_{e}^{p}$. Stochastic Processes and Their Applications, 21, 305-317.

[6] R. Dahlhaus (1989). Efficient parameter estimation of self-similar processes. Annals of Statistics, 17, 1749-1766.

[7] Y. Davydov (1970). The invariance principle for stationary processes. Theory of Probability and Its Applications., 15, 487-498.

[8] R. Dmowka, B. Saltzman eds. (1999). Long-Range Persistence in Geophysical Time Series. Advances in Geophysics, 40, Academic Press.

[9] R.L. Dobrushin and P. Major (1979). Non central limit theorems for non-linear functionals of Gaussian fields. Z. Wahrsch. verw. Geb., 50, 27-52.

[10] P. Doukhan, G. Oppenhaim, and M.S. Taqqu, eds. (2003). Theory and applications of long-range dependence. Birkhäuser, Boston. 
[11] P. Embrechts and M. Maejima (2002). Selfsimilar Processes. Princeton University Press. Oxford, Princeton.

[12] W. Feller (1951). The asymptotic distribution of the range of sums of independent random variables. Annals of Mathematical Statistics, 22, 427-432.

[13] J. Geweke and S. Porter-Hudak (1983). The estimation and application of long memory time series models. Journal of Time Series Analysis, Vol. 4, 4, 221-238.

[14] L. Giraitis and D. Surgailis (1985). CLT and other limit theorems for functionals of Gaussian processes. Z. Wahrsch. vew. Geb., 70, 191-212.

[15] L. Giraitis, P. Kokoszka, and R. Leipus (2000). Rescaled variance and related tests for long memory in volatility and levels. Preprint, Utah State University.

[16] L. Giraitis, P. Kokoszka, R. Leipus, and G. Teyssière (2003). Rescaled variance and related tests for long memory in volatility and levels. Journal of Econometrics, 112, 256-294.

[17] L. Giraitis, R. Leipus, and A. Philippe (2006). A test for stationarity versus trends and unit roots for a wide class of dependent errors. Journal of Econometric Theory, 22, 989-1029.

[18] U. Grenader and G. Szegö (1958). Toeplitz forms and their applications. Calif. Mono. in Math. Sci., Univ. of Calif. Press, Berkley and Los Angeles.

[19] H. E. Hurst (1951). Long-term storage capacity of reservoirs. Trans. Am. Soc. Civil Eng., 116, 770.

[20] Yu. I. Ingster and I. A. Suslina (2002). Nonparametric Goodness-Of-Fit Testing Under Gaussian Models. Springer-Verlag. 
[21] J. Kiefer (1959). K-sample analogues of the Kolmogorov-Smirnov and Cramér-v. Mises tests. Annals of Mathematical Statistics, 30, 420-447.

[22] H.R. Künsch (1987). Statistical aspects of self-similar processes. In Proceedings of the First World Congress of the Bernoulli Society (Yu. Prohorov and V.V. Sazonov, eds.), 1, 67-74. VNU Science Press, Utrecht.

[23] D. Kwiatkowski, P.Phillips, P.Scmidt and Y.Shin (1992). Testing the null hypothesis of stationarity against the alternative of a unit root. Journal of Econometrics, 54, 159-178.

[24] D. Lee and P. Schmidt (1996). On the power of the KPSS test of stationarity against fractionally-integrated alternatives. Journal of Econometrics, 73, 285302.

[25] H.S. Lee and C. Amsler (1997). Consistency of the KPSS unit root test against fractionally integrated alternative. Economics Letters, 55, 151-160.

[26] A.W. Lo (1991). Long-Term Memory in Stock Market Prices. Econometrica, 59, $1279-1313$.

[27] B.B. Mandelbrot and J. R. Wallis (1969). Computer experiments with fractional Gaussian noises, Parts 1, 2, and 3. Water Resources Res., 5(1), 228-267.

[28] B.B. Mandelbrot (1975). Limit theorems on the self-normalized range for weakly and strongly dependent processes. Zeishrift für Wahrscheinlichkeitstheorie und Verwandte Gebiete, 31(4), 271-285.

[29] W. Palma (2007). Long-Memory Time Series: Theory and Methods. John Wiley \& Sons, Inc., Hoboken, New Jersey. 
[30] P.M. Robinson (1995). Gaussian semiparametric estimation of long range dependence. Annals of Statistics, 13, 1630-1661.

[31] M.Rosenblatt (1952). Limit theorems associated with variants of the von Mises statistic. Annals of Mathematical Statistics, 23, 617-623.

[32] A. K. Md. Ehsanes Saleh (2006). Theory of preliminary test and Stein-type estimation with applications. John Wiley \& Sons.

[33] D. Surgailis (1982). Zones of attraction of self similar multiple integrals. Lithuanian Mathematical Journal, 22, 327-340.

[34] D. Surgailis, G. Teyssière, and M. Vaičiulis (2008). The increment ratio statistic. Journal of Multivariate Analysis, 99, 510-541.

[35] M.S. Taqqu (1979). Convergence of integrated processes of arbitrary Hermite rank. Z. Wahrsch. verw. Geb., 50, 53-83.

[36] G.S. Watson (1961). Goodness-of-fit tests on a circle. Biometrika, 48, 109-114. 